Equações diferenciais funcionais com retardamento e impulsos em tempo variável via equações diferenciais ordinárias generalizadas

Suzete Maria Silva Afonso 
SERVIÇO DE PÓS-GRADUAÇÃO DO ICMC-USP

Data de Depósito: 16/03/2011

Assinatura:

\title{
Equações diferenciais funcionais com retardamento e impulsos em tempo variável via equações diferenciais ordinárias generalizadas
}

\author{
Suzete Maria Silva Afonso \\ Orientadora: Profa. Dra. Márcia Cristina Anderson Braz Federson \\ Coorientador: Prof. Dr. Everaldo de Mello Bonotto
}

Tese apresentada ao Instituto de Ciências Matemáticas e de Computação - ICMC-USP, como parte dos requisitos para obtenção do título de Doutor em Matemática. VERSÃO REVISADA.

USP - São Carlos

Março de 2011 
Ficha catalográfica elaborada pela Biblioteca Prof. Achille Bassi e Seção Técnica de Informática, ICMC/USP, com os dados fornecidos pelo(a) autor(a)

Maria Silva Afonso, Suzete
Equaçóes diferenciais funcionais com retardamento
e impulsos em tempo variável via equaçoses
diferenciais ordinárias generalizadas / Suzete Maria
Silva Afonso; orientadora Márcia Cristina Anderson
Braz Federson -- São Carlos, 2011.
120 p.
Tese (Doutorado - Programa de Pós-Graduação em
Matemática) -- Instituto de Ciências Matemáticas e
de Computação, Universidade de São Paulo, 2011.
1. Equações Diferenciais Funcionais. 2. Equações
Diferenciais Ordinárias Generalizadas. I. Cristina
Anderson Braz Federson, Márcia, orient. II. Título.


Às pessoas mais importantes da minha vida, Humberto, Vanda e Odila (em memória), papai, mamãe e "vozinha". 



\section{Agradecimentos}

Minha incomparável gratidão ao soberano Deus por despertar em mim o amor pela matemática e a vontade de ser professora a cada manhã... Pela força visível durante cada minuto de apreensão e dificuldade... Pelo amor que me faz acreditar num futuro que vai além do que eu me limitei a sonhar para $\operatorname{mim}$.

Agradeço aos meus pais, Humberto e Vanda, por apoiarem as minhas escolhas, por entenderem a distância que se faz necessária para o meu crescimento profissional, pelas orações, pelos telefonemas, pelas visitas e pelas recepções amorosas na minha sempre casa... Por cada palavra de incentivo, pela paciência infinita e pela credibilidade que faz a minha vontade de ser melhor a cada dia tender a $+\infty$. A minha saudosa e incrível avó, Odila, agradeço pelo exemplo de mulher forte e determinada que me deixou e pela educação que me proporcionou em vida.

Agradeço aos meus irmãos, Humberto e Soraya, pelo incentivo sempre, pelo carinho a cada encontro, pela música e por cuidarem dos nossos pais. Ao meu sobrinho, Bento, agradeço por cada espera na janela e pelos "beijos da sorte" que foram mais do que especiais durante esse tempo do Doutorado. A tia Cicina, sou grata por ter me incentivado a estudar desde a infância.

Agradeço aos meus orientadores, profa. Dra. Márcia Federson e prof. Dr. Everaldo Bonotto, pela valiosa contribuição na minha formação, pelas palavras de apoio e de incentivo e, também, pelas broncas construtivas. Além disso, agradeço-vos pela paciência e pela amizade que fizeram a diferença neste período. Gostaria de registrar também a minha gratidão pela profa. Dra. Luciene Gimenes da Universidade Estadual de Maringá, por ter me acompanhado numa parte deste trabalho, dando-me apoio científico e até emocional.

Agradeço as minhas mais do que amigas MMIs, Jaqueline Rocha, Maria Eugênia, Mariana Pinheiro e Yuri $\mathrm{Ki}$, pelos reencontros regados de muitas risadas, pelos e-mails e telefonemas, pelas conversas no gtalk e no skype... Sou infinitamente grata pelo conjunto não enumerável de vezes em que pude contar com palavras e gestos de carinho de vocês. 
Agradeço aos amigos de Saquarema, de Campos dos Goytacazes e de Niterói, pela torcida, pelos retiros espirituais e pela força transmitida através de e-mails carinhosos, em especial, aos amigos, Adriana, Aluizio, Álvaro, Diego, Griseuda, Irmã Karol, Jefferson, Laura, Luciana, Luiz Alberto, Marcel, Reginaldo, Selene Ferreira, Thaís Barreto, Verônica e Vladimir.

Agradeço a cada membro do Grupo de Oração Universitário da USP, pelo apoio espiritual e pela amizade em Deus durante a minha estada em São Carlos. Em especial, agradeço aos amigos Evelise Corbalan, Fernando de Oliveira e Rita de Cássia pelo carinho inolvidável.

Agradeço aos amigos e colegas do ICMC, Alisson, Ana Paula, André Furtado, Cleber de Medeira, Eduard, Fernando Micena, Flank, Giselle, Iris, Jaque, Kleyber, Ligia, Marcos Pimenta, Nazira, Patricia Hilario, Paulo Carvalho, Pedro Henrique, Taciana e Vinicius Arakawa, pelas discussões matemáticas e não matemáticas, pelos momentos de descontração na USP e fora da USP e pelo companheirismo durante estes três anos de estudo.

Agradeço as amigas de (São Carlos - USP), Carolina Damasceno, Fátima, Flávia, Kelly, Raquel Parrelli, Sandrea e Selene, pela força e por ouvirem meus problemas do Doutorado com a maior paciência do mundo.

Agradeço aos professores doutores da Universidade Federal Fluminense, Jorge Delgado, Kátia Frensel e Nivaldo Medeiros, pelo incentivo inesquecível durante a graduação. Também agradeço ao prof. Dr. Nilson Bernardes da Universidade Federal do Rio de Janeiro, meu orientador durante o Mestrado, pela importante contribuição na minha vida acadêmica.

Finalmente, agradeço a FAPESP pelo suporte financeiro durante a execução deste trabalho. 


\section{Resumo}

O objetivo deste trabalho é investigar propriedades qualitativas das soluções de equações diferenciais funcionais com retardamento e impulsos em tempo variável (EDFRs impulsivas) através da teoria de equações diferenciais ordinárias generalizadas (EDOs generalizadas).

Nossos principais resultados dizem respeito a estabilidade uniforme, estabilidade uniforme assintótica e estabilidade exponencial da solução trivial de uma determinada classe de EDFRs com impulsos em tempo variável e limitação uniforme de soluções da mesma classe. A fim de obtermos tais resultados para EDFRs com impulsos em tempo variável, estabelecemos novos resultados sobre propriedades qualitativas das soluções de EDOs generalizadas. Assim, portanto, este trabalho contribui para o desenvolvimento de ambas as teorias de EDFRs com impulsos e de EDOs generalizadas.

Os resultados novos apresentados neste trabalho estão contidos nos artigos [1], [2] e [3]. 



\section{Abstract}

The purpose of this work is to investigate qualitative properties of solutions of retarded functional differential equations (RFDEs) with impulse effects acting on variable times using the theory of generalized ordinary differential equations (generalized ODEs).

Our main results concern uniform stability, uniform asymptotic stability and exponential stability of the trivial solution of a certain class of RFDEs with variable impulses and uniform boundedness of the solutions of the same class. In order to obtain such results for RFDEs with variable impulses, we establish new results about qualitative properties of solutions of generalized ODEs. In this manner, we contribute with new results not only to the theory of RFDEs with impulses but also to the theory of generalized ODEs.

The new results presented in this work are contained in the articles [1], [2] and [3]. 



\section{Sumário}

Introdução

1 Equações Diferenciais Ordinárias Generalizadas

$1.1 \quad$ A Integral de Kurzweil $\ldots \ldots \ldots \ldots \ldots$

1.2 Noções básicas de EDOs generalizadas . . . . . . . . . . . . . . . . . . . . . . . . . 19

2 Propriedades qualitativas de soluções de EDOs generalizadas 33

2.1 Estabilidade variacional da solução trivial . . . . . . . . . . . . . . . . . . . . . 33

2.2 Estabilidade exponencial variacional da solução trivial $\ldots \ldots \ldots$. . . . . . . . . . . 42

2.3 Limitação uniforme de soluções $\ldots \ldots \ldots$. . . . . . . . . . . . . . . . . . . 44

3 EDFRs com impulsos em tempo variável e EDOs generalizadas 49

3.1 Descrição da classe de EDFRs impulsivas $\ldots \ldots \ldots$

3.2 Correspondência entre EDFRs impulsivas e EDOs generalizadas . . . . . . . . . . 53

4 Propriedades qualitativas de soluções de EDFRs impulsivas via EDOs generalizadas 71

4.1 Estabilidade da solução trivial $\ldots \ldots \ldots \ldots \ldots$

4.1 .1 Comentários e observações . . . . . . . . . . . . . . . . . . . . . 87

4.2 Estabilidade exponencial da solução trivial $\ldots \ldots \ldots \ldots$

4.3 Limitação uniforme de soluções $\ldots \ldots \ldots \ldots \ldots$. . . . . . . . . . . . . . . 91

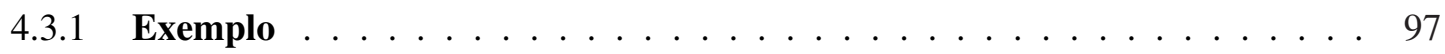

5 Apêndice: Propriedades qualitativas de soluções de EDFRs 101 
5.1 Noções e resultados básicos . . . . . . . . . . . . . . . . . . . . . . . . . . . . . 101

5.2 Estabilidade de soluções . . . . . . . . . . . . . . . . . . . . . . . . . . . . . . . . . . 104

5.2.1 Teoremas de Lyapunov . . . . . . . . . . . . . . . . . . . . . . . . . . . . 105

5.2 .2 Teoremas de Razumikhin . . . . . . . . . . . . . . . . . . . . . . . . 107

5.3 Limitação de soluções . . . . . . . . . . . . . . . . . . . . . . . . . . . . . . . . . . . 109 


\section{Introdução}

A teoria das equações diferenciais funcionais com retardamento (EDFRs) é um ramo das equações diferenciais funcionais. O interesse em EDFRs está em que, para muitos fenômenos naturais, notadamente físicos e biológicos, a aplicação do princípio de causalidade envolve um lapso de tempo entre causa e efeito. Desta forma, os modelos determinísticos mais realistas são frequentemente descritos por equações que envolvem retardos.

Paralelamente ao estudo das EDFRs, estamos interessados nos efeitos impulsivos sobre a dinâmica dos modelos que vamos estudar. Os impulsos representam variações do estado em lapsos de tempo tão pequenos que podem ser consideradas instantâneas. Estas variações correspondem às descontinuidades de primeira espécie das soluções ou de suas derivadas.

Neste trabalho, vamos considerar problemas como o seguinte problema de valor inicial

$$
\begin{cases}\dot{y}(t)=f\left(t, y_{t}\right), & t \neq \tau_{k}(y(t)), \quad t \geq t_{0}, \\ \Delta y(t)=I_{k}(y(t)), & t=\tau_{k}(y(t)), \quad k=1,2, \ldots \\ y_{t_{0}}=\phi, & \end{cases}
$$

onde $t_{0} \geq 0$ e a função inicial $\phi$ é regrada e contínua à esquerda, definida em $[-r, 0]$, com $r>0$. Consideraremos que, para cada solução $y:\left[t_{0}-r,+\infty\right) \rightarrow \mathbb{R}^{n}$ de (I), a aplicação $t \mapsto f\left(t, y_{t}\right)$ é localmente Lebesgue integrável. Vamos considerar, também, que os operadores de impulso $I_{k}(x), k=0,1,2, \ldots$ sejam funções contínuas de $\mathbb{R}^{n}$ em $\mathbb{R}^{n}$ e que

$$
\Delta y(t)=y(t+)-y(t-)=y(t+)-y(t)=I_{k}(y(t)), \quad k=0,1,2, \ldots,
$$

isto é , y é contínua à esquerda.

Vamos assumir condições do tipo "Carathéodory" e do tipo "Lipschitz" para a integral indefinida de $f$ e para os operadores de impulsos $I_{k}$. Grosseiramente falando, condições impostas sobre a integral 
indefinida e não sobre a função $f$ propriamente dita permitem, por exemplo, que a função $f$ tenha muitas descontinuidades e, mesmo assim, é possível obtermos bons resultados, desde que sua integral indefinida tenha um "bom comportamento". Isto pode ser feito, pois podemos tratar as EDFRs num "ambiente" apropriado dentro de uma classe de equações diferenciais ordinárias (EDOs) generalizadas (veja [10] e [27]).

Em relação às superfícies $\tau_{k}, k=1,2, \ldots$, vamos considerar que $\tau_{k} \in C\left(\mathbb{R}^{n},(0,+\infty)\right), k=1,2, \ldots$, $0<\tau_{1}(x)<\tau_{2}(x)<\ldots$, para cada $x \in \mathbb{R}^{n}, \tau_{k}(x) \rightarrow+\infty$ quando $k \rightarrow+\infty$ uniformemente em $x \in \mathbb{R}^{n}$, e que as curvas integrais do sistema (I) encontram tais superfícies somente um número finito de vezes.

Note que o problema impulsivo acima trata de impulsos variáveis e, portanto, não conhecemos de antemão os momentos onde ocorrerão os saltos das soluções pelos operadores de impulso.

Neste trabalho, vamos investigar propriedades qualitativas das soluções de EDFRs com impulsos em tempo variável, através da teoria das EDOs generalizadas usando, também, funcionais de Lyapunov.

Inicialmente, no Capítulo 1, apresentamos a teoria de EDOs generalizadas necessária para obtermos os nossos resultados, enquanto que, no Capítulo 2, estudamos propriedades qualitativas das soluções de EDOs generalizadas, a saber estabilidade variacional e estabilidade exponencial variacional da solução nula de uma determinada classe de EDOs generalizadas, e limitação uniforme de soluções da mesma classe. Convém mencionarmos que os conceitos de estabilidade exponencial variacional e limitação uniforme para EDOs generalizadas são novos e foram por nós introduzidos nos trabalhos [1] e [3], assim como os resultados obtidos nesta direção.

No terceiro capítulo, descrevemos uma classe de EDFRs com impulsos em tempo variável para a qual pretendemos investigar propriedades qualitativas das soluções. Prosseguimos, no Capítulo 3, mostrando como se dá a correspondência entre esta classe de EDFRs impulsivas e uma classe de EDOs generalizadas. Na verdade, esta correspondência se dá entre a solução única de um problema de valor inicial para EDFRs impulsivas e a solução única de um problema de valor inicial para a EDO generalizada associada. Para impulsos pré-fixados, tal correspondência pode ser encontrada em [8]. Aqui, nós estendemos a correspondência para EDFRs com impulsos em tempo variável e EDOs generalizadas. Esta correspondência nos permitiu obter resultados mais gerais do que os existentes na literatura.

No Capítulo 4, estudamos propriedades qualitativas das soluções da classe de EDFRs com impulsos descrita no Capítulo 3. Estabelecemos novos resultados sobre estabilidade uniforme e estabilidade assintótica uniforme para a solução nula da classe de EDFRs com impulsos que consideramos. Tais resultados generalizam outros. Por exemplo, nosso Teorema 4.9 generaliza o Teorema 1 de [25]. O Teorema 4.10, 
na sequência, generaliza [25], Teorema 3, [26], Teorema 1, e também [22], Teorema 3.2. Também, realizamos um estudo sobre estabilidade exponencial da solução trivial da classe de EDFRs com impulsos e, inspirados no artigo [11], estabelecemos um novo resultado que generaliza os Corolários 3.1 e 3.2 daquele artigo. Finalizamos o Capítulo 4 apresentando resultados sobre limitação uniforme e limitação eventual uniforme de soluções de EDFRs com impulsos, que generalizam os resultados de [29], por exemplo. Mencionamos que os resultados deste capítulo estão descritos nos artigos [1], [2] e [3].

Incluímos, no apêndice deste trabalho, fatos conhecidos da teoria de EDFRs, como resultados sobre propriedades qualitativas das soluções, a fim de que o leitor perceba as diferenças entre tais resultados e os resultados obtidos para a teoria de EDFRs com ação impulsiva. 



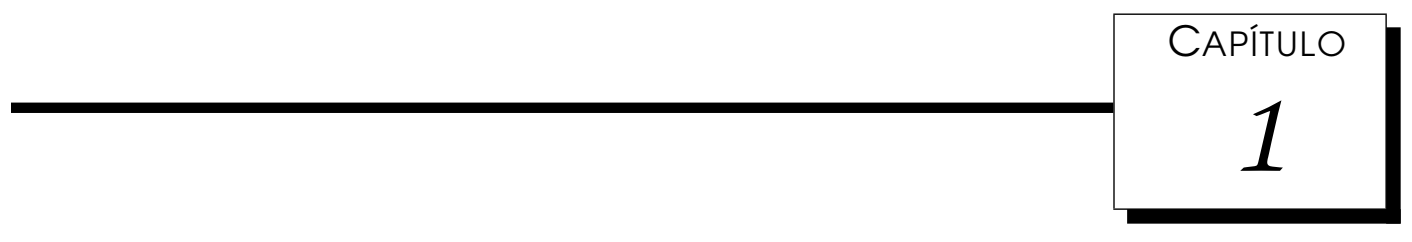

\section{Equações Diferenciais Ordinárias Generalizadas}

Neste capítulo, introduziremos algumas definições básicas e apresentaremos alguns resultados importantes sobre EDOs generalizadas que serão de grande valia ao longo do trabalho.

Dividimos este capítulo em duas seções. A primeira seção será dedicada à teoria básica da integral de Kurzweil. Na segunda seção, apresentaremos as fundamentais EDOs generalizadas, que são definidas a partir da integral de Kurzweil.

As principais referências para este capítulo são [12] e [27].

\section{$1.1 \quad$ A Integral de Kurzweil}

Consideremos um intervalo fechado $[a, b] \subset \mathbb{R}$, onde $-\infty<a<b<+\infty$.

Uma divisão marcada do intervalo $[a, b] \subset \mathbb{R}$ é uma coleção finita

$$
\left\{\left(\tau_{j},\left[s_{j-1}, s_{j}\right]\right): j=1,2, \ldots, k\right\}
$$

onde $a=s_{0} \leq s_{1} \leq \ldots \leq s_{k}=b$ é uma divisão de $[a, b]$ e $\tau_{j} \in\left[s_{j-1}, s_{j}\right], j=1,2, \ldots, k$.

Uma divisão parcial marcada do intervalo $[a, b] \subset \mathbb{R}$ é uma coleção finita

$$
\left\{\left(\xi_{j},\left[\beta_{j}, \gamma_{j}\right]\right): j=1,2, \ldots, m\right\}
$$


onde $a \leq \beta_{j} \leq \xi_{j} \leq \gamma_{j} \leq \beta_{j+1} \leq \xi_{j+1} \leq \gamma_{j+1} \leq b$, para $j=1, \ldots, m$.

Note que toda divisão marcada é uma divisão parcial marcada.

Um calibre em $[a, b]$ é qualquer função $\delta:[a, b] \rightarrow(0,+\infty)$. Dado um calibre $\delta$ em $[a, b]$, uma divisão parcial marcada $d=\left(\tau_{j},\left[\beta_{j}, \gamma_{j}\right]\right)$ de $[a, b]$ será dita $\delta$-fina se, para todo $j$, tivermos

$$
\left[\beta_{j}, \gamma_{j}\right] \subset\left\{t \in[a, b]:\left|t-\tau_{j}\right|<\delta\left(\tau_{j}\right)\right\}
$$

No que segue, $X$ denotará um espaço de Banach e $\|\cdot\|$ denotará sua norma.

Agora, apresentaremos a definição da integral de Kurzweil, devida ao matemático tcheco Jaroslav Kurzweil (veja [21]).

Definição 1.1. Uma função $U:[a, b] \times[a, b] \rightarrow X$ será dita Kurzweil integrável sobre $o$ intervalo $[a, b]$, se existir um elemento $I \in X$ tal que para todo $\varepsilon>0$, existe um calibre $\delta$ em $[a, b]$ tal que

$$
\left\|\sum_{j=1}^{k}\left[U\left(\tau_{j}, s_{j}\right)-U\left(\tau_{j}, s_{j-1}\right)\right]-I\right\|<\varepsilon
$$

para toda divisão marcada $\delta$-fina

$$
d=\left\{\left(\tau_{j},\left[s_{j-1}, s_{j}\right]\right), j=1, \ldots, k\right\}=\left\{s_{0}, \tau_{1}, s_{1}, \ldots, \tau_{k}, s_{k}\right\}
$$

de $[a, b]$. Neste caso, escrevemos $I=\int_{a}^{b} D U(\tau, t)$. Definimos $\int_{a}^{b} D U(\tau, t)=-\int_{b}^{a} D U(\tau, t)$, quando $b<a, e \int_{a}^{b} D U(\tau, t)=0$, quando $b=a$. A unicidade da integral é fácil de ser verificada.

Cabe mencionar que, no Capítulo 1 de [27], há uma descrição detalhada deste tipo de integração para o caso em que $X=\mathbb{R}^{n}$.

O próximo resultado trata da integrabilidade em subintervalos de $[a, b]$, a qual também é uma propriedade das integrais de Bochner e de Riemann.

Teorema 1.2 ([27], Teorema 1.10). Se $U:[a, b] \times[a, b] \rightarrow X$ for Kurzweil integrável sobre $[a, b]$, então a integral $\int_{c}^{d} D U(\tau, t)$ existirá, para cada subintervalo $[c, d] d e[a, b]$.

Outras propriedades das integrais de Bochner e de Riemann também valem para a integral de Kurzweil, como a propriedade usual de aditividade em subintervalos adjacentes. Vejamos o próximo resultado cuja prova encontra-se em [27], Teorema 1.11. 
Teorema 1.3 ([27], Teorema 1.11). Sejam $c \in(a, b)$ e $U:[a, b] \times[a, b] \rightarrow X$ uma função. Se as integrais $\int_{a}^{c} D U(\tau, t)$ e $\int_{c}^{b} D U(\tau, t)$ existirem, então a integral $\int_{a}^{b} D U(\tau, t)$ existirá e a igualdade

$$
\int_{a}^{b} D U(\tau, t)=\int_{a}^{c} D U(\tau, t)+\int_{c}^{b} D U(\tau, t)
$$

será válida.

O resultado que enunciaremos abaixo é conhecido como Lema de Saks-Henstock. Uma prova deste pode ser encontrada em [27], Lemma 1.13, para $X=\mathbb{R}^{n}$. A demonstração para $X$ sendo um espaço de Banach arbitrário é análoga.

Lema 1.4 (Saks-Henstock - [27], Lema 1.13). Seja $U:[a, b] \times[a, b] \rightarrow X$ Kurzweil integrável sobre $[a, b]$. Dado $\varepsilon>0$, seja $\delta$ uma função calibre em $[a, b]$ tal que

$$
\left\|\sum_{j=1}^{k}\left[U\left(\tau_{j}, s_{j}\right)-U\left(\tau_{j}, s_{j-1}\right)\right]-\int_{a}^{b} D U(\tau, t)\right\|<\varepsilon
$$

para toda divisão marcada $\delta$-fina $d=\left\{\left(\tau_{j},\left[s_{j-1}, s_{j}\right]\right), j=1,2, \ldots, k\right\}$ de $[a, b]$. Se

$$
a \leq \beta_{1} \leq \xi_{1} \leq \gamma_{1} \leq \beta_{2} \leq \xi_{2} \leq \gamma_{2} \leq \ldots \leq \beta_{m} \leq \xi_{m} \leq \gamma_{m} \leq b
$$

representar uma divisão parcial marcada $\delta$-fina $\left\{\left(\xi_{j},\left[\beta_{j}, \gamma_{j}\right]\right), j=1,2, \ldots, m\right\}$ de $[a, b]$, isto é,

$$
\xi_{j} \in\left[\beta_{j}, \gamma_{j}\right] \subset\left(\xi_{j}-\delta\left(\xi_{j}\right), \xi_{j}+\delta\left(\xi_{j}\right)\right), \quad j=1,2, \ldots, m,
$$

então teremos

$$
\left\|\sum_{j=1}^{m}\left[U\left(\xi_{j}, \beta_{j}\right)-U\left(\xi_{j}, \gamma_{j}\right)-\int_{\beta_{j}}^{\gamma_{j}} D U(\tau, t)\right]\right\|<\varepsilon .
$$

Como consequência do Lema de Saks-Henstock, temos o resultado abaixo.

Corolário 1.5. Seja $U:[a, b] \times[a, b] \rightarrow X$ Kurzweil integrável sobre $[a, b]$. Dado $\varepsilon>0$, seja $\delta$ a função calibre em $[a, b]$ da Definição 1.1. Seja $[\gamma, v]$ um subintervalo fechado de $[a, b]$. Então,

(i) $(v-\gamma)<\delta(\gamma)$ implica

$$
\left\|U(\gamma, v)-U(\gamma, \gamma)-\int_{\gamma}^{v} D U(\tau, t)\right\|<\varepsilon
$$

(ii) $(v-\gamma)<\delta(v)$ implica

$$
\left\|U(v, v)-U(v, \gamma)-\int_{\gamma}^{v} D U(\tau, t)\right\|<\varepsilon
$$


Demonstração. (i) Segue do fato que $(\gamma,[\gamma, v])$ é uma divisão marcada $\delta$-fina de $[\gamma, v]$ e do Lema de Saks-Henstock. Um argumento análogo se aplica a (ii).

O próximo teorema diz que a integral de Kurzweil contém suas extensões de Cauchy (integrais impróprias). Para maiores detalhes, veja [16], página 67, e também [27] .

Teorema 1.6 ([27], Teorema 1.14). Se $U:[a, b] \times[a, b] \rightarrow X$ for uma função tal que para todo $c \in[a, b)$, $U$ é Kurzweil integrável sobre $[a, c]$ e o limite

$$
\lim _{c \rightarrow b-}\left[\int_{a}^{c} D U(\tau, t)-U(b, c)+U(b, b)\right]=I \in X
$$

existir, então a função U será Kurzweil integrável sobre $[a, b]$ e teremos

$$
\int_{a}^{b} D U(\tau, t)=I
$$

Analogamente, se a função $U$ for Kurzweil integrável sobre $[c, b]$ para todo $c \in(a, b]$ e o limite

$$
\lim _{c \rightarrow a+}\left[\int_{c}^{b} D U(\tau, t)+U(a, c)-U(a, a)\right]=I \in X
$$

existir, então a função U será Kurzweil integrável sobre $[a, b]$ e teremos

$$
\int_{a}^{b} D U(\tau, t)=I
$$

Se $U:[a, b] \times[a, b] \rightarrow X$ for Kurzweil integrável sobre $[a, b]$, a função definida por

$$
s \in[a, b] \longmapsto \int_{a}^{s} D U(\tau, t) \in X
$$

ou seja, a integral indefinida de $U$ poderá não ser contínua. Veja o próximo lema.

Lema 1.7 ([27], Teorema 1.16). Sejam $U:[a, b] \times[a, b] \rightarrow X$ uma função Kurzweil integrável sobre $[a, b]$ $e c \in[a, b]$. Então

$$
\lim _{s \rightarrow c}\left[\int_{a}^{s} D U(\tau, t)-U(c, s)+U(c, c)\right]=\int_{a}^{c} D U(\tau, t)
$$

$e$

$$
\lim _{s \rightarrow c}\left[\int_{s}^{b} D U(\tau, t)-U(c, s)+U(c, c)\right]=\int_{c}^{b} D U(\tau, t) .
$$

O próximo resultado generaliza o Teorema 1.35 encontrado em [27], para o caso das funções a 
valores em espaços de Banach. Para uma prova, veja [12], Teorema 1.13.

Teorema 1.8 ([12], Teorema 1.13). Seja $U:[a, b] \times[a, b] \rightarrow X$ uma função Kurzweil integrável sobre $[a, b]$. Se $V:[a, b] \times[a, b] \rightarrow \mathbb{R}$ for Kurzweil integrável sobre $[a, b]$ e se existir uma função calibre $\theta$ em $[a, b]$ tal que

$$
|t-\tau|\|U(\tau, t)-U(\tau, \tau)\| \leq(t-\tau)[V(\tau, t)-V(\tau, \tau)]
$$

para todo $t \in(\tau-\theta(\tau), \tau+\theta(\tau))$, então a desigualdade

$$
\left\|\int_{a}^{b} D U(\tau, t)\right\| \leq \int_{a}^{b} D V(\tau, t)
$$

será válida.

\subsection{Noções básicas de EDOs generalizadas}

Vamos considerar um conjunto $\Omega=O \times[a, b]$, onde $O$ é um subconjunto aberto de $X$ e $[a, b] \subset \mathbb{R}$.

Seja $G: \Omega \rightarrow X$ uma função a valores em $X$ definida para $(x, t) \in \Omega$. Lembramos ao leitor que estamos considerando $X$ como sendo um espaço de Banach.

Definição 1.9. Uma função $x:[\alpha, \beta] \rightarrow X$ será dita solução da EDO generalizada

$$
\frac{d x}{d \tau}=D G(x, t)
$$

no intervalo $[\alpha, \beta] \subset[a, b]$, se $(x(t), t) \in \Omega$ para todo $t \in[\alpha, \beta]$ e se a igualdade

$$
x\left(s_{2}\right)-x\left(s_{1}\right)=\int_{s_{1}}^{s_{2}} D G(x(\tau), t)
$$

for válida para quaisquer $s_{1}, s_{2} \in[\alpha, \beta]$.

A integral do lado direito da igualdade em (1.6) deve ser entendida como sendo a integral de Kurzweil.

Observação 1.10. Com a notação da Definição 1.9, seja $U(\tau, t)=G(x(\tau), t)$. Observe que, na definição da integral $\int_{a}^{b} D G(x(\tau), t)$ (Definição 1.1), existem somente diferenças da forma

$$
U\left(\tau_{j}, s_{j}\right)-U\left(\tau_{j}, s_{j-1}\right)=G\left(x\left(\tau_{j}\right), s_{j}\right)-G\left(x\left(\tau_{j}\right), s_{j-1}\right),
$$


onde $d=\left(\tau_{j},\left[s_{j-1}, s_{j}\right]\right)$ é uma divisão marcada $\delta$-fina do intervalo $[a, b]$. Por conseguinte, se adicionarmos a $G(x, t)$ uma função variando somente em $x$, as soluções de (1.5) não mudarão. De fato, note que, dada uma função $F: X \rightarrow X$, temos

$$
\begin{gathered}
\int_{a}^{b} D[G(x(\tau), t)-F(x(\tau))]=\sum\left[\left(G\left(x\left(\tau_{j}\right), s_{j}\right)-F\left(x\left(\tau_{j}\right)\right)\right)-\left(G\left(x\left(\tau_{j}\right), s_{j-1}\right)-F\left(x\left(\tau_{j}\right)\right)\right)\right]= \\
=\sum\left[G\left(x\left(\tau_{j}\right), s_{j}\right)-G\left(x\left(\tau_{j}\right), s_{j-1}\right)\right]=\int_{a}^{b} D G(x(\tau), t) .
\end{gathered}
$$

Desta forma, se $x:[\alpha, \beta] \rightarrow X$ for solução da EDO generalizada

$$
\frac{d x}{d \tau}=D[G(x, t)+F(x)]
$$

então x será solução da EDO generalizada (1.5) no intervalo $[\alpha, \beta]$. E a afirmação recíproca também se verifica. Em particular, subtraindo $G(x, 0)$ de $G(x, t)$, obtemos uma representação normalizada $G_{1}$ de $G$ satisfazendo $G_{1}(x, 0)=0$, para todo $x \in O$.

Dada uma condição inicial $\left(\widetilde{x}, t_{0}\right) \in \Omega$, usaremos a seguinte definição de solução de um problema de valor inicial para a equação (1.5).

Definição 1.11. Uma função $x:[\alpha, \beta] \rightarrow X$ será dita solução da EDO generalizada (1.5) com condição inicial $x\left(t_{0}\right)=\widetilde{x}$ no intervalo $[\alpha, \beta] \subset[a, b]$, se $t_{0} \in[\alpha, \beta],(x(t), t) \in \Omega$ para todo $t \in[\alpha, \beta]$ e se a igualdade

$$
x(v)-\widetilde{x}=\int_{t_{0}}^{v} D G(x(\tau), t)
$$

for válida para todo $v \in[\alpha, \beta]$.

A seguir, introduziremos uma classe especial de funções $G: \Omega \rightarrow X$ para a qual será possível obter informações mais específicas sobre as soluções da EDO generalizada (1.5). Convém mencionarmos que, devido à relevância dos resultados existentes a respeito de soluções da classe de EDOs generalizadas relacionada à essa classe de funções, exibiremos as demonstrações dos resultados expostos nesta subseção.

Suporemos, ao longo deste capítulo, que $h$ seja uma função real não decrescente definida em $[a, b]$.

Definição 1.12. Diremos que uma função $G: \Omega \rightarrow X$ pertence à classe $\mathscr{F}(\Omega, h)$, se

$$
\left\|G\left(x, t_{2}\right)-G\left(x, t_{1}\right)\right\| \leq\left|h\left(t_{2}\right)-h\left(t_{1}\right)\right|
$$


para quaisquer $\left(x, t_{2}\right),\left(x, t_{1}\right) \in \Omega$ e se

$$
\left\|G\left(x, t_{2}\right)-G\left(x, t_{1}\right)-G\left(y, t_{2}\right)+G\left(y, t_{1}\right)\right\| \leq\|x-y\|\left|h\left(t_{2}\right)-h\left(t_{1}\right)\right|
$$

para quaisquer $\left(x, t_{2}\right),\left(x, t_{1}\right),\left(y, t_{2}\right),\left(y, t_{1}\right) \in \Omega$.

O próximo resultado generaliza o Lema 3.9 de [27] para o caso em que funções assumem valores em $X$.

Lema 1.13 ([27], Lema 3.9). Suponha que $G: \Omega \rightarrow X$ satisfaça a condição (1.8). Se $[\alpha, \beta] \subset[a, b] e$ $x:[\alpha, \beta] \rightarrow X$ for tal que $(x(t), t) \in \Omega$ para todo $t \in[\alpha, \beta]$ e se a integral $\int_{\alpha}^{\beta} D G(x(\tau), t)$ existir, então para quaisquer $s_{1}, s_{2} \in[\alpha, \beta]$, a desigualdade

$$
\left\|\int_{s_{1}}^{s_{2}} D G(x(\tau), t)\right\| \leq\left|h\left(s_{2}\right)-h\left(s_{1}\right)\right|
$$

será válida.

Demonstração. Pela condição (1.8),

$$
|t-\tau||| G(x(\tau), t)-G(x(\tau), \tau) \| \leq|t-\tau||h(t)-h(\tau)|
$$

para quaisquer $t, \tau \in[\alpha, \beta]$.

Tomando $V(\tau, t)=h(t)$, a integral $\int_{\alpha}^{\beta} D V(\tau, t)=\int_{\alpha}^{\beta} d h(t)$ existe no sentido de Lebesgue e vale

$$
\int_{s_{1}}^{s_{2}} d h(t)=h\left(s_{2}\right)-h\left(s_{1}\right)
$$

para quaisquer $s_{1}, s_{2} \in[\alpha, \beta]$. Pelo Teorema 1.8 , obtemos a seguinte relação

$$
\left\|\int_{s_{1}}^{s_{2}} D G(x(\tau), t)\right\| \leq\left|\int_{s_{1}}^{s_{2}} D V(\tau, t)\right| .
$$

Portanto,

$$
\left\|\int_{s_{1}}^{s_{2}} D G(x(\tau), t)\right\| \leq\left|h\left(s_{2}\right)-h\left(s_{1}\right)\right|
$$

para quaisquer $s_{1}, s_{2} \in[\alpha, \beta]$.

As definições seguintes serão utilizadas ao longo deste capítulo. 
Definição 1.14. Uma função $f:[a, b] \rightarrow \mathbb{R}$ será dita função escada finita, se existir uma divisão finita $a=\beta_{0}<\beta_{1}<\ldots<\beta_{m}=b$ tal que em cada intervalo aberto $\left(\beta_{i-1}, \beta_{i}\right), i=1,2, \ldots, m$, a função é identicamente igual a uma constante $c_{i} \in \mathbb{R}$.

Definição 1.15. Uma função $f:[a, b] \rightarrow \mathbb{R}$ será dita função regrada no intervalo $[a, b]$, se os limites laterais $f(s-)=\lim _{\rho \rightarrow 0-} f(s+\rho)$ e $f(s+)=\lim _{\rho \rightarrow 0+} f(s+\rho)$ existirem para todo $s \in(a, b]$ e $s \in[a, b)$, respectivamente.

É sabido que toda função regrada em $[a, b]$ é limitada neste intervalo e é o limite uniforme de funções escada finitas. É sabido, também, que toda função de variação limitada em $[a, b]$ é regrada em $[a, b]$. Veja [17], por exemplo.

O próximo resultado implicará que as soluções de (1.5) são de variação limitada, se $G$ satisfizer a condição (1.8).

Lema 1.16 ([27], Lema 3.10). Suponha que $G: \Omega \rightarrow X$ satisfaça a condição (1.8). Se $[\alpha, \beta] \subset[a, b] e$ $x:[\alpha, \beta] \rightarrow X$ for uma solução de (1.5), então valerá a desigualdade

$$
\left\|x\left(s_{1}\right)-x\left(s_{2}\right)\right\| \leq\left|h\left(s_{2}\right)-h\left(s_{1}\right)\right|
$$

para quaisquer $s_{1}, s_{2} \in[\alpha, \beta]$.

Demonstração. O resultado segue diretamente do Lema 1.13 e da definição de solução da EDO generalizada, Definição 1.9 .

Denotaremos a variação em $[\alpha, \beta] \subset \mathbb{R}$ de uma função $x: \mathbb{R} \rightarrow X$ por $v^{\beta} r_{\alpha}^{\beta} x$. O Lema 1.16 implica a seguinte propriedade das soluções de (1.5).

Corolário 1.17 ([27], Corolário 3.11). Suponha que $G: \Omega \rightarrow X$ satisfaça a condição (1.8). Se $[\alpha, \beta] \subset$ $(a, b)$ e $x:[\alpha, \beta] \rightarrow X$ for uma solução de (1.5), então x será de variação limitada em $[\alpha, \beta]$ e

$$
\operatorname{var}_{\alpha}^{\beta} x \leq h(\beta)-h(\alpha)<+\infty
$$

Além disso, todo ponto em $[\alpha, \beta]$ no qual a função hé contínua também será um ponto de continuidade da solução $x:[\alpha, \beta] \rightarrow X$. 
Demonstração. Consideremos $\alpha=s_{0}<s_{1}<\ldots<s_{k}=\beta$ uma divisão arbitrária do intervalo $[\alpha, \beta]$. A desigualdade (1.11) implica que

$$
\sum_{j=1}^{k}\left\|x\left(s_{j}\right)-x\left(s_{j-1}\right)\right\| \leq \sum_{j=1}^{k}\left[h\left(s_{j}\right)-h\left(s_{j-1}\right)\right]=h(\beta)-h(\alpha),
$$

uma vez que todas as hipóteses do Lema 1.16 estão satisfeitas. Passando o supremo sobre todas as divisões de $[\alpha, \beta]$, obtemos (1.12).

Claramente, a segunda afirmação segue da desigualdade (1.11).

Também temos o resultado seguinte.

Lema 1.18 ([27], Lema 3.12). Se $x:[\alpha, \beta] \rightarrow X$ for uma solução de (1.5) $e G: \Omega \rightarrow X$ satisfizer a condição (1.8), então

$$
x(\sigma+)-x(\sigma)=\lim _{s \rightarrow \sigma+} x(s)-x(\sigma)=G(x(\sigma), \sigma+)-G(x(\sigma), \sigma)
$$

para $\sigma \in[\alpha, \beta) e$

$$
x(\sigma)-x(\sigma-)=x(\sigma)-\lim _{s \rightarrow \sigma-} x(s)=G(x(\sigma), \sigma)-G(x(\sigma), \sigma-)
$$

para $\sigma \in(\alpha, \beta]$, onde

$$
G(x, \sigma+)=\lim _{s \rightarrow \sigma+} G(x, s), \quad \text { para } \sigma \in[\alpha, \beta)
$$

$e$

$$
G(x, \sigma-)=\lim _{s \rightarrow \sigma-} G(x, s), \quad \text { para } \sigma \in(\alpha, \beta] .
$$

Demonstração. A função $h$ possui limites laterais em todos os pontos do intervalo $[\alpha, \beta]$, visto que $h$ é de variação limitada em $[\alpha, \beta]$. Pela condição (1.8), os limites $G(x, \sigma+)$ e $G(x, \sigma-)$ existem. Portanto, os limites laterais correspondentes da função $G(x, \sigma):[\alpha, \beta] \rightarrow X$ existem para todo $x \in O$.

Pela definição de uma solução de (1.5), temos a igualdade abaixo

$$
x(\sigma)-x(s)=\int_{s}^{\sigma} D G(x(\tau), t)
$$


para $\alpha \leq s<\sigma \leq \beta$. Pelo Teorema 1.7, temos

$$
\lim _{\sigma \rightarrow s^{+}} x(\sigma)-x(s)=\lim _{\sigma \rightarrow s^{+}} \int_{\sigma}^{s} D G(x(\tau), t)=\lim _{\sigma \rightarrow s^{+}}[G(x(s), \sigma)-G(x(s), s)]
$$

e a relação (1.13) está satisfeita.

A prova da relação (1.14) segue de forma análoga.

O próximo lema garante a existência da integral $\int_{\alpha}^{\beta} D G(x(\tau), t)$ quando $x$ for uma função escada finita.

Lema 1.19 ([27], Corolário 3.15). Se $G \in \mathscr{F}(\Omega, h)$ e $x:[\alpha, \beta] \rightarrow X$ for uma função escada finita, então existirá a integral

$$
\int_{\alpha}^{\beta} D G(x(\tau), t)
$$

Demonstração. Se $x$ for uma função escada finita, então existirá uma divisão $\alpha=s_{0}<s_{1}<\ldots<s_{k}=\beta$ de $[\alpha, \beta]$ tal que $x(s)=c_{j} \in X$ para $s \in\left(s_{j-1}, s_{j}\right), j=1,2, \ldots, k$ onde $c_{j}$ é constante para todo $j=$ $1,2, \ldots, k$. Suponhamos que $(x(s), s) \in \Omega$ para todo $s \in[\alpha, \beta]$. Pela definição da integral de Kurzweil, é claro que se $s_{j-1}<\sigma_{1}<\sigma_{2}<s_{j}$, a integral $\int_{\sigma_{1}}^{\sigma_{2}} D G(x(\tau), t)$ existirá e

$$
\int_{\sigma_{1}}^{\sigma_{2}} D G(x(\tau), t)=G\left(c_{j}, \sigma_{2}\right)-G\left(c_{j}, \sigma_{1}\right)
$$

Tomemos $\sigma_{0} \in\left(s_{j-1}, s_{j}\right)$. Temos

$$
\begin{aligned}
& \lim _{s \rightarrow s_{j-1}^{+}}\left[\int_{s}^{\sigma_{0}} D G(x(\tau), t)+G\left(x\left(s_{j-1}\right), s\right)-G\left(x\left(s_{j-1}\right), s_{j-1}\right)\right]= \\
= & \lim _{s \rightarrow s_{j-1}^{+}}\left[G\left(c_{j}, \sigma_{0}\right)-G\left(c_{j}, s\right)+G\left(x\left(s_{j-1}\right), s\right)-G\left(x\left(s_{j-1}\right), s_{j-1}\right)\right]= \\
= & G\left(c_{j}, \sigma_{0}\right)-G\left(c_{j}, s_{j-1}+\right)+G\left(x\left(s_{j-1}\right), s_{j-1}+\right)-G\left(x\left(s_{j-1}\right), s_{j-1}\right) .
\end{aligned}
$$

Portanto, pelo Teorema 1.6, a integral $\int_{s_{j-1}}^{\sigma_{0}} D G(x(\tau), t)$ existe e é igual ao limite calculado acima.

Analogamente, podemos constatar que a integral $\int_{\sigma_{0}}^{s_{j}} D G(x(\tau), t)$ existe e vale

$$
\int_{\sigma_{0}}^{s_{j}} D G(x(\tau), t)=G\left(c_{j}, s_{j}-\right)-G\left(c_{j}, \sigma_{0}\right)-G\left(x\left(s_{j}\right), s_{j}-\right)+G\left(x\left(s_{j}\right), s_{j}\right) .
$$


Portanto, pelo Teorema 1.3, obtemos a existência da integral $\int_{s_{j-1}}^{s_{j}} D G(x(\tau), t) \mathrm{e}$

$$
\begin{gathered}
\int_{s_{j-1}}^{s_{j}} D G(x(\tau), t)=G\left(c_{j}, s_{j}-\right)-G\left(c_{j}, \sigma_{0}\right)-G\left(x\left(s_{j}\right), s_{j}-\right)+G\left(x\left(s_{j}\right), s_{j}\right)+ \\
+G\left(c_{j}, \sigma_{0}\right)-G\left(c_{j}, s_{j-1}+\right)+G\left(x\left(s_{j-1}\right), s_{j-1}+\right)-G\left(x\left(s_{j-1}\right), s_{j-1}\right)= \\
=G\left(c_{j}, s_{j}-\right)-G\left(c_{j}, s_{j-1}+\right)+G\left(x\left(s_{j-1}\right), s_{j-1}+\right)- \\
-G\left(x\left(s_{j-1}\right), s_{j-1}\right)-G\left(x\left(s_{j}\right), s_{j}-\right)+G\left(x\left(s_{j}\right), s_{j}\right) .
\end{gathered}
$$

Repetindo este argumento para todo intervalo $\left[s_{j-1}, s_{j}\right], j=1,2, \ldots, k$, e usando novamente o Teorema 1.3, obtemos a existência da integral $\int_{\alpha}^{\beta} D G(x(\tau), t)$ como também a igualdade

$$
\begin{gathered}
\int_{\alpha}^{\beta} D G(x(\tau), t)=\sum_{j=1}^{k}\left[G\left(c_{j}, s_{j}-\right)-G\left(c_{j}, s_{j-1}+\right)\right]+ \\
+\sum_{j=1}^{k}\left[G\left(x\left(s_{j-1}\right), s_{j-1}+\right)-G\left(x\left(s_{j-1}\right), s_{j-1}\right)-G\left(x\left(s_{j}\right), s_{j}-\right)+G\left(x\left(s_{j}\right), s_{j}\right)\right] .
\end{gathered}
$$

Deste modo, a prova está completa.

A seguir, provaremos a existência da integral $\int_{\alpha}^{\beta} D G(x(\tau), t)$ quando $x$ for uma função regrada.

Teorema 1.20 ([12], Teorema 1.26). Seja $G \in \mathscr{F}(\Omega, h)$. Se $x:[\alpha, \beta] \rightarrow X$, com $[\alpha, \beta] \subset[a, b]$, for o limite uniforme de uma sequência $\left(x_{k}\right)_{k \in \mathbb{N}}$ de funções escada finitas $x_{k}:[\alpha, \beta] \rightarrow X$ tais que $(x(s), s) \in \Omega$ $e\left(x_{k}(s), s\right) \in \Omega$ para todo $k \in \mathbb{N}$ e todo $s \in[\alpha, \beta]$, então a integral $\int_{\alpha}^{\beta} D G(x(\tau), t)$ existirá e a igualdade

$$
\int_{\alpha}^{\beta} D G(x(\tau), t)=\lim _{k \rightarrow \infty} \int_{\alpha}^{\beta} D G\left(x_{k}(\tau), t\right)
$$

será válida.

Demonstração. Para cada $k \in \mathbb{N}$, a integral $\int_{\alpha}^{\beta} D G\left(x_{k}(\tau), t\right)$ existe, pelo Lema 1.19.

Dado $\varepsilon>0$, seja $k_{0} \in \mathbb{N}$ tal que, para $k \geq k_{0}$, tenhamos

$$
\left\|x_{k}(s)-x(s)\right\|<\frac{\varepsilon}{2[h(\beta)-h(\alpha)]}, \quad s \in[\alpha, \beta],
$$


e seja $\delta$ um calibre em $[a, b]$ tal que, para $k \geq k_{0}$, tenhamos

$$
\left\|\sum_{i=1}^{m}\left[G\left(x_{k}\left(\tau_{i}\right), t_{i}\right)-G\left(x_{k}\left(\tau_{i}\right), t_{i-1}\right)\right]-\int_{\alpha}^{\beta} D G\left(x_{k}(\tau), t\right)\right\|<\frac{\varepsilon}{2}
$$

para toda divisão marcada $\delta$-fina $d=\left\{t_{0} \leq \tau_{1} \leq t_{1} \leq \ldots \leq t_{m-1} \leq \tau_{m} \leq \beta\right\}$ de $[\alpha, \beta]$. Então para todo $k \geq k_{0}$, vale

$$
\begin{gathered}
\left\|\sum_{i=1}^{m}\left[G\left(x\left(\tau_{i}\right), t_{i}\right)-G\left(x\left(\tau_{i}\right), t_{i-1}\right)\right]-\int_{\alpha}^{\beta} D G\left(x_{k}(\tau), t\right)\right\| \leq \\
\leq \sum_{i=1}^{m}\left\|G\left(x\left(\tau_{i}\right), t_{i}\right)-G\left(x\left(\tau_{i}\right), t_{i-1}\right)-G\left(x_{k}\left(\tau_{i}\right), t_{i}\right)+G\left(x_{k}\left(\tau_{i}\right), t_{i-1}\right)\right\|+ \\
+\left\|\sum_{i=1}^{m}\left[G\left(x_{k}\left(\tau_{i}\right), t_{i}\right)-G\left(x_{k}\left(\tau_{i}\right), t_{i-1}\right)\right]-\int_{\alpha}^{\beta} D G\left(x_{k}(\tau), t\right)\right\| \leq \\
\leq \sum_{i=1}^{m}\left[h\left(t_{i}\right)-h\left(t_{i-1}\right)\right] \max _{1 \leq i \leq n}\left\|x\left(\tau_{i}\right)-x_{k}\left(\tau_{i}\right)\right\|+\frac{\varepsilon}{2}= \\
=[h(\beta)-h(\alpha)] \max _{1 \leq i \leq n}\left\|x\left(\tau_{i}\right)-x_{k}\left(\tau_{i}\right)\right\|+\frac{\varepsilon}{2}<\frac{\varepsilon}{2}+\frac{\varepsilon}{2}=\varepsilon,
\end{gathered}
$$

o que finaliza a prova.

O resultado abaixo é uma consequência do Teorema 1.20 para funções regradas e generaliza o Corolário 3.16 encontrado em [27], para o caso de funções a valores em espaços de Banach.

Corolário 1.21 ([27], Corolário 3.16). Se $G \in \mathscr{F}(\Omega, h)$ e $x:[\alpha, \beta] \rightarrow X,[\alpha, \beta] \subset[a, b]$, for uma função regrada (em particular, uma função de variação limitada) em $[\alpha, \beta]$ tal que $(x(s), s) \in \Omega$ para todo $s \in[\alpha, \beta]$, então a integral

$$
\int_{\alpha}^{\beta} D G(x(\tau), t)
$$

existirá.

Demonstração. Toda função regrada é o limite uniforme de funções escada finitas. Logo todas as hipóteses do Teorema 1.20 estão satisfeitas, donde segue o resultado.

Na sequência, apresentaremos um resultado que trata da existência local e unicidade de soluções de (1.5) com condição inicial. Veremos que a prova deste decorre do Teorema do Ponto Fixo de Banach, também conhecido como Teorema da Contração Uniforme (veja [19], página 300), que será enunciado abaixo. 
Teorema 1.22 (Teorema do Ponto Fixo de Banach). Seja X um espaço de Banach com norma $\|\cdot\|_{X}$. Se uma aplicação $f: X \rightarrow X$ for uma contração uniforme, ou seja, se existir $0 \leq \beta<1$ tal que

$$
\|f(x)-f(y)\|_{X} \leq \beta\|x-y\|_{X}, \quad \text { para quaisquer } x, y \in X,
$$

então existirá um único ponto $x^{*} \in X$ tal que

$$
f\left(x^{*}\right)=x^{*}
$$

Teorema 1.23 (Existência e Unicidade - [8], Teorema 2.15). Seja $G: \Omega \rightarrow X$ uma função pertencente à classe $F(\Omega, h)$, onde $h:[a, b] \rightarrow \mathbb{R}$ é uma função não decrescente e contínua à esquerda em $(a, b]$. Então para todo $\left(\widetilde{x}, t_{0}\right) \in \Omega$ satisfazendo

$$
\widetilde{x}+=\widetilde{x}+G\left(\widetilde{x}, t_{0}+\right)-G\left(\widetilde{x}, t_{0}\right) \in O,
$$

teremos $\left(\widetilde{x}+, t_{0}\right) \in \Omega$ e existirá um $\Delta>0$ tal que, no intervalo $\left[t_{0}, t_{0}+\Delta\right]$, existirá uma única solução $x:\left[t_{0}, t_{0}+\Delta\right] \rightarrow X$ da EDO generalizada (1.5) satisfazendo $x\left(t_{0}\right)=\tilde{x}$.

Demonstração. Inicialmente, suponhamos que $t_{0}$ seja um ponto de continuidade da função $h$, ou seja, que $h\left(t_{0}+\right)=h\left(t_{0}\right)$. Suponhamos que $\Delta>0$ seja tal que $\left[t_{0}, t_{0}+\Delta\right] \subset[a, b], h\left(t_{0}+\Delta\right)-h\left(t_{0}\right)<\frac{1}{2}$ e $\|x-\widetilde{x}\| \leq\left|h(t)-h\left(t_{0}\right)\right|$ implica que $x \in O$, para $t \in\left[t_{0}, t_{0}+\Delta\right]$.

Denotaremos o espaço de Banach das funções de variação limitada $z:\left[t_{0}, t_{0}+\Delta\right] \rightarrow X$ por $B V\left(\left[t_{0}, t_{0}+\right.\right.$ $\Delta], X)$ com a norma usual da variação dada por

$$
\|z\|_{B V}=\left\|z\left(t_{0}\right)\right\|+v a r_{t_{0}}^{t_{0}+\Delta} z
$$

Seja $Q$ o conjunto das funções $z:\left[t_{0}, t_{0}+\Delta\right] \rightarrow X$ tais que $z \in B V\left(\left[t_{0}, t_{0}+\Delta\right], X\right)$ e $\|z(t)-\widetilde{x}\| \leq$ $\left|h(t)-h\left(t_{0}\right)\right|$, para $t \in\left[t_{0}, t_{0}+\Delta\right]$. Vamos mostrar que $Q$ é fechado. Para isso, consideremos $\left\{z_{n}\right\}_{n \in \mathbb{N}} \subset Q$ uma sequência tal que $z_{n} \rightarrow z^{*}$. Como

$$
\left\|z_{n}-z^{*}\right\|_{B V}=\left\|z_{n}\left(t_{0}\right)-z^{*}\left(t_{0}\right)\right\|+v a r_{t_{0}}^{t_{0}+\Delta}\left(z_{n}-z^{*}\right),
$$

e $\left\|z_{n}(t)-z^{*}(t)\right\| \leq\left\|z_{n}-z^{*}\right\|_{B V}$, para qualquer $t \in\left[t_{0}, t_{0}+\Delta\right]$, concluímos que $z_{n} \rightarrow z^{*}$ uniformemente. 
Portanto, dado $\varepsilon>0$, existe $n \in \mathbb{N}$ tal que

$$
\left\|z^{*}(t)-\widetilde{x}\right\| \leq\left\|z^{*}(t)-z_{n}(t)\right\|+\left\|z_{n}(t)-\widetilde{x}\right\| \leq \varepsilon+\left|h(t)-h\left(t_{0}\right)\right| .
$$

Assim $\left\|z^{*}(t)-\widetilde{x}\right\| \leq\left|h(t)-h\left(t_{0}\right)\right|$ para qualquer $t \in\left[t_{0}, t_{0}+\Delta\right]$ e, por conseguinte, $z^{*} \in Q$, o que implica que $Q$ é fechado.

Como $B V\left(\left[t_{0}, t_{0}+\Delta\right], X\right)$ é um espaço de Banach e $Q \subset B V\left(\left[t_{0}, t_{0}+\Delta\right], X\right)$ é fechado, constatamos que $Q$ é um espaço de Banach.

Para $s \in\left[t_{0}, t_{0}+\Delta\right]$ e $z \in Q$, definamos

$$
T z(s)=\widetilde{x}+\int_{t_{0}}^{s} D G(z(\tau), t)
$$

A integral $\int_{t_{0}}^{s} D G(z(\tau), t)$ existe pelo fato de $z$ ser de variação limitada e $G$ pertencer à classe $\mathscr{F}(\Omega, h)$ (veja o Corolário 1.21). Então, pelo Lema 1.13, vale

$$
\|T z(s)-\widetilde{x}\|=\left\|\int_{t_{0}}^{s} D G(z(\tau), t)\right\| \leq\left|h(s)-h\left(t_{0}\right)\right|
$$

Portanto, $T z \in Q$, isto é, $T$ aplica $Q$ em $Q$.

Agora, provemos que $T$ é uma contração. Tomemos uma divisão finita $t_{0}-\Delta=\alpha_{0}<\alpha_{1}<\ldots<$ $\alpha_{i-1}<\alpha_{i}<\ldots<\alpha_{k}=t_{0}+\Delta$ do intervalo $\left[t_{0}, t_{0}+\Delta\right], z_{1}, z_{2} \in Q$ e $\varepsilon>0$. Então, para $i \in\{1, \ldots, k\}$, temos

$$
\begin{gathered}
\left\|T z_{2}\left(\alpha_{i}\right)-T z_{1}\left(\alpha_{i}\right)-\left[T z_{2}\left(\alpha_{i-1}\right)-T z_{1}\left(\alpha_{i-1}\right)\right]\right\|=\| \int_{t_{0}}^{\alpha_{i}} D\left[G\left(z_{2}(\tau), t\right)-G\left(z_{1}(\tau), t\right)\right] \\
-\int_{t_{0}}^{\alpha_{i-1}} D\left[G\left(z_{2}(\tau), t\right)-G\left(z_{1}(\tau), t\right)\right]\|=\| \int_{\alpha_{i-1}}^{\alpha_{i}} D\left[G\left(z_{2}(\tau), t\right)-G\left(z_{1}(\tau), t\right)\right] \| \\
=\| \int_{\alpha_{i}}^{\alpha_{i-1}} D\left[G\left(z_{2}(\tau), t\right)-G\left(z_{1}(\tau), t\right)\right]-\sum_{j=1}^{n}\left[G\left(z_{2}\left(\tau_{j}\right), s_{j}\right)-G\left(z_{1}\left(\tau_{j}\right), s_{j}\right)-G\left(z_{2}\left(\tau_{j}\right), s_{j-1}\right)\right. \\
\left.+G\left(z_{1}\left(\tau_{j}\right), s_{j-1}\right)\right]+\sum_{j=1}^{n}\left[G\left(z_{2}\left(\tau_{j}\right), s_{j}\right)-G\left(z_{1}\left(\tau_{j}\right), s_{j}\right)-G\left(z_{2}\left(\tau_{j}\right), s_{j-1}\right)+G\left(z_{1}\left(\tau_{j}\right), s_{j-1}\right)\right] \| \\
\leq\left\|\sum_{j=1}^{n}\left[G\left(z_{2}\left(\tau_{j}\right), s_{j}\right)-G\left(z_{1}\left(\tau_{j}\right), s_{j}\right)-G\left(z_{2}\left(\tau_{j}\right), s_{j-1}\right)+G\left(z_{1}\left(\tau_{j}\right), s_{j-1}\right)\right]\right\|+\varepsilon \\
\leq \sum_{j=1}^{n}\left\|z_{2}\left(\tau_{j}\right)-z_{1}\left(\tau_{j}\right)\right\|\left[h\left(s_{j}\right)-h\left(s_{j-1}\right)\right]+\varepsilon
\end{gathered}
$$




$$
\begin{gathered}
\leq \sup _{\tau \in\left[\alpha_{i-1}, \alpha_{i}\right]}\left\|z_{2}(\tau)-z_{1}(\tau)\right\|\left(\sum_{j=1}^{n}\left[h\left(s_{j}\right)-h\left(s_{j-1}\right)\right]\right)+\varepsilon \\
\leq \sup _{\tau \in\left[t_{0}, t_{0}+\Delta\right]}\left\|z_{2}(\tau)-z_{1}(\tau)\right\|\left[h\left(\alpha_{i}\right)-h\left(\alpha_{i-1}\right)\right]+\varepsilon,
\end{gathered}
$$

para qualquer divisão marcada $\delta$-fina $\left\{\left(\tau_{j},\left[s_{j-1}, s_{j}\right]\right), j=1,2, \ldots, n\right\}$ de $\left[\alpha_{i-1}, \alpha_{i}\right]$.

Como $\varepsilon$ é arbitrário, temos

$$
\begin{gathered}
\left\|T z_{2}\left(\alpha_{i}\right)-T z_{1}\left(\alpha_{i}\right)-\left[T z_{2}\left(\alpha_{i-1}\right)-T z_{1}\left(\alpha_{i-1}\right)\right]\right\| \leq \\
\quad \leq \sup _{\tau \in\left[t_{0}, t_{0}+\Delta\right]}\left\|z_{2}(\tau)-z_{1}(\tau)\right\|\left[h\left(\alpha_{i}\right)-h\left(\alpha_{i-1}\right)\right] .
\end{gathered}
$$

Logo,

$$
v a r_{t_{0}}^{t_{0}+\Delta}\left(T z_{2}-T z_{1}\right) \leq \sup _{\tau \in\left[t_{0}, t_{0}+\Delta\right]}\left\|z_{2}(\tau)-z_{1}(\tau)\right\| v a r_{t_{0}}^{t_{0}+\Delta} h
$$

Mas

$$
\sup _{\tau \in\left[t_{0}, t_{0}+\Delta\right]}\left\|z_{2}(\tau)-z_{1}(\tau)\right\| \leq \operatorname{var}_{t_{0}}^{t_{0}+\Delta}\left(z_{2}-z_{1}\right) .
$$

Logo, por (1.16) e (1.17), temos

$$
\begin{gathered}
\operatorname{var}_{t_{0}}^{t_{0}+\Delta}\left(\operatorname{Tz}_{2}-T z_{1}\right) \leq \operatorname{var}_{t_{0}}^{t_{0}+\Delta}\left(z_{2}-z_{1}\right) \operatorname{var}_{t_{0}}^{t_{0}+\Delta} h= \\
=\operatorname{var}_{t_{0}}^{t_{0}+\Delta}\left(z_{2}-z_{1}\right)\left[h\left(t_{0}+\Delta\right)-h\left(t_{0}\right)\right] .
\end{gathered}
$$

Pela definição de $Q$, segue imediatamente que se $z_{i} \in Q, i=1,2$, então $z_{i}\left(t_{0}\right)=\widetilde{x}$. Temos, também, a igualdade $T\left(z_{i}\left(t_{0}\right)\right)=\tilde{x}, i=1,2$.

Logo (1.18) implica que

$$
\left\|T z_{2}-T z_{1}\right\|_{B V} \leq\left\|z_{2}-z_{1}\right\|_{B V}\left[h\left(t_{0}+\Delta\right)-h\left(t_{0}\right)\right]<\frac{1}{2}\left\|z_{2}-z_{1}\right\|_{B V} .
$$

Portanto, pelo Teorema do Ponto Fixo de Banach, temos o resultado desejado.

Agora, consideremos $t_{0}$ como sendo um ponto de descontinuidade de $h$ e definamos

$$
\widetilde{h}(t)=\left\{\begin{array}{l}
h(t), \quad \text { se } t \leq t_{0} \\
h(t)-\left(h\left(t_{0}+\right)-h\left(t_{0}\right)\right), \quad \text { se } t>t_{0} .
\end{array}\right.
$$


Então a função $\widetilde{h}$ é contínua em $t_{0}$, não decrescente e contínua à esquerda em $t>t_{0}$.

Definindo, também,

$$
\widetilde{G}(x, t)=\left\{\begin{array}{l}
G(x, t), \quad \text { se } t \leq t_{0}, \\
G(x, t)-\left(G\left(\widetilde{x}, t_{0}+\right)-G\left(\widetilde{x}, t_{0}\right)\right), \quad \text { se } t>t_{0},
\end{array}\right.
$$

temos $\widetilde{G} \in \mathscr{F}(\Omega, \widetilde{h})$. Portanto, realizando um procedimento análogo ao anterior, concluímos que existe uma solução $z$ de $\frac{d z}{d \tau}=D \widetilde{G}(z(\tau), t) \operatorname{com} z\left(t_{0}\right)=\widetilde{x}+$. Por conseguinte, definindo

$$
x(t)= \begin{cases}\widetilde{x}, & \text { se } t=t_{0}, \\ z(t), & \text { se } t>t_{0},\end{cases}
$$

notamos que $x$ é solução de $\frac{d x}{d \tau}=D G(x, t) \operatorname{com} x\left(t_{0}\right)=\widetilde{x}$ (veja Observação 1.10) e a prova está completa.

Definiremos, a seguir, o conceito de continuação à direita de uma solução de (1.5).

Definição 1.24. Se x for uma solução de (1.5) em um intervalo $\left[t_{0}, t_{1}\right]$, com $\left[t_{0}, t_{1}\right] \subset[a, b]$, diremos que $z$ é uma continuação à direita de $x$, se existir $t_{2}>t_{1}\left(t_{2}<b\right)$ de modo que zesteja definida em $\left[t_{0}, t_{2}\right]$ e se $z$ coincidir com $x$ em $\left[t_{0}, t_{1}\right]$ e satisfizer a equação $(1.5)$ em $\left[t_{0}, t_{2}\right]$.

Vejamos que a continuação à direita de uma solução de (1.5) está fundamentada no lema abaixo.

Lema 1.25 ([27], Lema 4.4). Se $x:[\alpha, \beta] \rightarrow X$ e $y:[\beta, \gamma] \rightarrow X$ forem soluções da equação (1.5) em $[\alpha, \beta]$ e em $[\beta, \gamma]$, respectivamente, com $[\alpha, \gamma] \subset[a, b]$ e $x(\beta)=y(\beta)$, então a função $z:[\alpha, \gamma] \rightarrow X$ definida pelas relações $z(s)=x(s)$ para $s \in[\alpha, \beta]$ e $z(s)=y(s)$ para $s \in[\beta, \gamma]$ será uma solução da equação (1.5).

Demonstração. Pelo Teorema 1.3, para $s_{1}, s_{2} \in[\alpha, \gamma], s_{1}<\beta<s_{2}$, temos

$$
\begin{aligned}
z\left(s_{2}\right)-z\left(s_{1}\right) & =z\left(s_{2}\right)-z(\beta)+z(\beta)-z\left(s_{1}\right) \\
& =y\left(s_{2}\right)-y(\beta)+x(\beta)-x\left(s_{1}\right) \\
& =\int_{\beta}^{s_{2}} D G(y(\tau), t)+\int_{s_{1}}^{\beta} D G(x(\tau), t) \\
& =\int_{s_{1}}^{s_{2}} D G(z(\tau), t) .
\end{aligned}
$$


Claramente, isto prova o resultado.

Observemos que, como estamos considerando a possibilidade de (1.5) admitir solução descontínua, pode acontecer o fato de que, para algum $\widetilde{x} \in O$, ou seja, para algum $\left(\widetilde{x}, t_{0}\right) \in \Omega$, o valor

$$
\widetilde{x}+=\widetilde{x}+G\left(\widetilde{x}, t_{0}+\right)-G\left(\widetilde{x}, t_{0}\right)
$$

não pertença à $O$. Por essa razão, foi necessário acrescentar a hipótese de que

$$
\widetilde{x}+=\widetilde{x}+G\left(\widetilde{x}, t_{0}+\right)-G\left(\widetilde{x}, t_{0}\right) \in O
$$

no Teorema 1.23.

Agora, vamos definir o conjunto

$$
\mathscr{G}=\{(x, t) \in \Omega:(x+G(x, t+)-G(x, t), t) \in \Omega\} .
$$

Consideremos a solução $x:\left[t_{0}, t_{0}+\Delta\right] \rightarrow X$ da EDO generalizada (1.5) no intervalo $\left[t_{0}, t_{0}+\Delta\right] \subset[a, b]$ tal que $x\left(t_{0}\right)=\widetilde{x}$. A solução $x$ admitirá uma continuação à direita, se $\left(\left(x\left(t_{0}+\Delta\right)\right)+, t_{0}+\Delta\right)$ pertencer ao conjunto $\mathscr{G}$. Este fato provém do Teorema 1.23. Portanto, se $x:\left[t_{0}, t_{1}\right] \rightarrow X$ for uma solução de (1.5) em $\left[t_{0}, t_{1}\right] \subset[a, b]$ tal que $(x(s), s) \in \mathscr{G}$ para todo $s \in\left[t_{0}, t_{1}\right]$, então $x$ poderá ser prolongada à direita (para valores $s>t_{1}$ ) à uma solução de (1.5). Sendo assim, diremos que $x:\left[t_{0}, t_{1}\right] \rightarrow X$ é uma solução não continuável à direita, se $\left(x\left(t_{1}\right), t_{1}\right) \notin \mathscr{G}$ ou $t_{1}=b$.

Para finalizar este capítulo, veremos o conceito de uma solução maximal de (1.5).

Definição 1.26. Diremos que $x:\left[t_{0}, t_{0}+\sigma\right) \rightarrow X, \sigma>0,\left[t_{0}, t_{0}+\sigma\right) \subset[a, b]$,é uma solução maximal de (1.5), com $x\left(t_{0}\right)=u \in O$, se $x$ for uma solução de (1.5) em todo intervalo $\left[t_{0}, t_{0}+\beta\right], \beta<\sigma$, e não puder ser prolongada ao intervalo $\left[t_{0}, t_{0}+\sigma\right]$.

Para um estudo mais abrangente sobre EDOs generalizadas, consulte [27]. 



\section{Propriedades qualitativas de}

\section{soluções de EDOs generalizadas}

Este capítulo será destinado ao estudo de algumas propriedades qualitativas de soluções de uma certa classe de EDOs generalizadas e será dividido em três seções. Na primeira seção, discutiremos a estabilidade variacional da solução nula de EDOs generalizadas. Na segunda seção, introduziremos o conceito de estabilidade exponencial variacional e daremos condições para que a solução nula de uma determinada classe de EDOs generalizadas seja variacionalmente exponencialmente estável. Finalmente, na terceira seção, estabeleceremos resultados sobre limitação uniforme de soluções de EDOs generalizadas.

As principais referências para este capítulo são [1], [2], [3], [27] e [28].

Neste capítulo, $X$ denotará um espaço de Banach com norma $\|\cdot\|$, assim como no capítulo anterior.

\subsection{Estabilidade variacional da solução trivial}

Nesta seção, vamos considerar $\Omega=B_{c} \times\left[t_{0},+\infty\right)$, onde $B_{c}=\{y \in X:\|y\|<c\}$, com $c>0$ e $t_{0} \geq 0$. Considararemos a EDO generalizada

$$
\frac{d x}{d \tau}=D G(x, t)
$$

onde $G \in \mathscr{F}(\Omega, h)$ e $h:\left[t_{0},+\infty\right) \rightarrow \mathbb{R}$ é uma função não decrescente e contínua à esquerda.

Vamos supor que $G(0, t)-G(0, s)=0$, para $t, s \geq t_{0}$. Então, para $[\gamma, v] \subset\left[t_{0},+\infty\right)$, temos

$$
\int_{\gamma}^{v} D G(0, t)=G(0, v)-G(0, \gamma)=0
$$


e, por conseguinte, $x \equiv 0$ é uma solução da EDO generalizada (2.1) em $\left[t_{0},+\infty\right)$. Note também que, pelo Corolário 1.17, toda solução de (2.1) é de variação limitada. Logo, é natural medir a distância entre duas soluções de (2.1) usando a norma da variação.

Os próximos conceitos sobre estabilidade foram introduzidos por Š. Schwabik em [28]. Veja também [27]. Tais conceitos levam em conta a variação das soluções de (2.1) em torno da solução nula $x \equiv 0$.

Definição 2.1. A solução trivial $x \equiv 0$ de (2.1) será dita

(i) Variacionalmente estável, se para todo $\varepsilon>0$, existir $\delta=\delta(\varepsilon)>0$ tal que se $\bar{x}:[\gamma, v] \rightarrow B_{c}$, $t_{0} \leq \gamma<v<+\infty$, for uma função de variação limitada em $[\gamma, v]$ e contínua à esquerda em $(\gamma, v]$ tal que

$$
\|\bar{x}(\gamma)\|<\delta
$$

$e$

$$
\operatorname{var}_{\gamma}^{\nu}\left(\bar{x}(s)-\int_{\gamma}^{s} D G(\bar{x}(\tau), t)\right)<\delta
$$

então

$$
\|\bar{x}(t)\|<\varepsilon, \quad t \in[\gamma, v]
$$

(ii) Variacionalmente atratora, se existir $\delta_{0}>0$ e para todo $\varepsilon>0$, existir $T=T(\varepsilon) \geq 0$ e $\rho=\rho(\varepsilon)>0$ tal que se $\bar{x}:[\gamma, v] \rightarrow B_{c}, t_{0} \leq \gamma<v<+\infty$, for uma função de variação limitada em $[\gamma, v]$ e contínua à esquerda em $(\gamma, v]$ tal que

$$
\|\bar{x}(\gamma)\|<\delta_{0}
$$

$e$

$$
\operatorname{var}_{\gamma}^{v}\left(\bar{x}(s)-\int_{\gamma}^{s} D G(\bar{x}(\tau), t)\right)<\rho
$$

então

$$
\|\bar{x}(t)\|<\varepsilon, \quad t \in[\gamma, v] \cap[\gamma+T,+\infty), \gamma \geq 0 .
$$

(iii) Variacionalmente assintoticamente estável, se for variacionalmente estável e variacionalmente atratora.

Observação 2.2. Note que, se $\bar{x}:[\gamma, v] \rightarrow X, t_{0} \leq \gamma<v<+\infty$, for uma solução de (2.1), então:

$$
\operatorname{var}_{\gamma}^{\nu}\left(\bar{x}(s)-\int_{\gamma}^{s} D G(\bar{x}(\tau), t)\right)=0
$$


De fato, como

$$
\bar{x}(s)-\bar{x}(\gamma)=\int_{\gamma}^{s} D G(\bar{x}(\tau), t), \quad s \in[\gamma, v]
$$

temos

$$
\operatorname{var}_{\gamma}^{\nu}\left(\bar{x}(s)-\int_{\gamma}^{s} D G(\bar{x}(\tau), t)\right)=\operatorname{var}_{\gamma}^{\nu}(\bar{x}(\gamma)-\bar{x}(\gamma))=0
$$

A seguir, apresentaremos o conceito de funcional de Lyapunov. Aqui, consideraremos $\mathbb{R}_{+}=\{z \in$ $\mathbb{R}: z \geq 0\}$.

Definição 2.3. Diremos que $V:\left[t_{0},+\infty\right) \times X \rightarrow \mathbb{R}$ é um funcional de Lyapunov (com respeito a EDO generalizada (2.1)), se as seguintes condições forem satisfeitas:

(i) $V(\cdot, x):\left[t_{0},+\infty\right) \rightarrow \mathbb{R}$ é contínuo à esquerda em $\left(t_{0},+\infty\right)$ para todo $x \in X$

(ii) Existe uma função monótona crescente $b: \mathbb{R}_{+} \rightarrow \mathbb{R}_{+}$tal que $b(0)=0 e$

$$
V(t, x) \geq b(\|x\|)
$$

para cada $t \in\left[t_{0},+\infty\right)$ e para cada $x \in X$;

(iii) Para toda solução $x:[\gamma, v] \rightarrow X$ de (2.1), $[\gamma, v] \subset\left[t_{0},+\infty\right)$,

$$
\dot{V}(t, x(t))=\limsup _{\eta \rightarrow 0+} \frac{V(t+\eta, x(t+\eta))-V(t, x(t))}{\eta} \leq 0,
$$

isto é, a derivada à direita de $V$ é não positiva ao longo de toda solução $x(t)$ de (2.1).

Agora, voltaremos a nossa atenção aos teoremas do tipo Lyapunov para a equação (2.1) (veja o Apêndice, seção 6.2.1). Inicialmente, porém, apresentaremos alguns resultados auxiliares.

Lema 2.4 ([27], Proposição 10.11). Sejam $-\infty<a<b<+\infty$ e $f, g:[a, b] \rightarrow \mathbb{R}$ funções contínuas à esquerda em $(a, b]$. Se para todo $\sigma \in[a, b]$, existir um $\delta(\sigma)$ tal que para todo $\eta \in(0, \delta(\sigma))$ a desigualdade

$$
g(\sigma+\eta)-g(\sigma) \leq f(\sigma+\eta)-f(\sigma)
$$

é válida, então teremos

$$
g(s)-g(a) \leq f(s)-f(a)
$$

para todo $s \in[a, b]$. 
Uma prova do lema enunciado acima pode ser encontrada em [27], Proposição 10.11.

O próximo resultado está presente em [27] (veja o Lema 10.12), para o caso em que a função $V$ está definida em $[0,+\infty) \times \mathbb{R}^{n}$. Aqui, porém, vamos considerar $V$ definida em $\left[t_{0},+\infty\right) \times X$. A fim de facilitarmos a compreensão do leitor, exibimos uma prova mais detalhada deste resultado seguindo as ideias de [27], Lema 10.12.

Lema 2.5. Seja $G \in \mathscr{F}(\Omega, h)$. Suponha que $V:\left[t_{0},+\infty\right) \times X \rightarrow \mathbb{R}$ seja tal que $V(\cdot, x):\left[t_{0},+\infty\right) \rightarrow \mathbb{R} e ́$ contínua à esquerda em $\left(t_{0},+\infty\right)$ para $x \in X$ e satisfaça

$$
|V(t, z)-V(t, y)| \leq K\|z-y\|, \quad z, y \in X, t \in\left[t_{0},+\infty\right)
$$

onde $K$ é uma constante positiva. Além disso, suponha que exista uma função $\Phi: X \rightarrow \mathbb{R}$ tal que para toda solução $x:[a, b] \rightarrow X,[a, b] \subset\left[t_{0},+\infty\right)$, de (2.1), tenhamos

$$
\dot{V}(t, x(t))=\limsup _{\eta \rightarrow 0+} \frac{V(t+\eta, x(t+\eta))-V(t, x(t))}{\eta} \leq \Phi(x(t)), \quad t \in[a, b] .
$$

Se $\bar{x}:[\gamma, v] \rightarrow X, t_{0} \leq \gamma<v<+\infty$, for contínua à esquerda em $(\gamma, v]$ e de variação limitada em $[\gamma, v]$, então

$$
V(v, \bar{x}(v))-V(\gamma, \bar{x}(\gamma)) \leq K v \operatorname{var}_{\gamma}^{v}\left(\bar{x}(s)-\int_{\gamma}^{s} D G(\bar{x}(\tau), t)\right)+M(v-\gamma),
$$

onde $M=\sup _{t \in[\gamma, v]} \Phi(\bar{x}(t))$.

Demonstração. Seja $\bar{x}:[\gamma, v] \rightarrow X$ uma função contínua à esquerda em $(\gamma, v]$ e de variação limitada em $[\gamma, v] \subset\left[t_{0},+\infty\right)$. Pelo Corolário 1.21, a integral $\int_{\gamma}^{v} D G(\bar{x}(\tau), t)$ existe.

Tomemos $\sigma \in[\gamma, v]$. Pelo Teorema 1.23, a equação (2.1) admite uma solução local, digamos, $x$ : $\left[\sigma, \sigma+\eta_{1}(\sigma)\right] \rightarrow X$ em $\left[\sigma, \sigma+\eta_{1}(\sigma)\right]$, satisfazendo a condição inicial $x(\sigma)=\bar{x}(\sigma)$. É claro que a integral

$\int_{\sigma}^{\sigma+\eta_{1}(\sigma)} D G(x(\tau), t)$ existe. Seja $\eta_{2}>0$ suficientemente pequeno tal que $\eta_{2} \leq \eta_{1}(\sigma)$ e $\sigma+\eta_{2} \leq v$. Então a integral $\int_{\sigma}^{\sigma+\eta_{2}} D G(x(\tau), t)$ existe e a integral $\int_{\sigma}^{\sigma+\eta_{2}} D[G(\bar{x}(\tau), t)-G(x(\tau), t)]$ também existe pelo Teorema 1.2. Portanto, dado $\varepsilon>0$, existe uma função calibre $\delta$ de $\left[\sigma, \sigma+\eta_{2}\right]$ correspondente a $\varepsilon$ na definição da última integral. Podemos supor, sem perda de generalidade, que $\eta_{2}<\delta(\sigma)$. Por (2.3), podemos tomar $0<\eta \leq \eta_{2}$ de forma que a desigualdade

$$
V(\sigma+\eta, x(\sigma+\eta))-V(\sigma, x(\sigma)) \leq \eta \Phi(x(\sigma))
$$


seja válida, e podemos supor, pelo Corolário 1.5(i), que

$$
\left\|G(\bar{x}(\sigma), \sigma+\eta)-G(\bar{x}(\sigma), \sigma)-\int_{\sigma}^{\sigma+\eta} D G(\bar{x}(\tau), t)\right\|<\frac{\eta \varepsilon}{2 K}
$$

$\mathrm{e}$

$$
\left\|G(x(\sigma), \sigma+\eta)-G(x(\sigma), \sigma)-\int_{\sigma}^{\sigma+\eta} D G(x(\tau), t)\right\|<\frac{\eta \varepsilon}{2 K}
$$

Note que

$$
\begin{gathered}
\left\|\int_{\sigma}^{\sigma+\eta} D[G(\bar{x}(\tau), t)-G(x(\tau), t)]\right\|-\|G(\bar{x}(\sigma), \sigma+\eta)-G(\bar{x}(\sigma), \sigma)-G(x(\sigma), \sigma+\eta)+G(x(\sigma), \sigma)\| \\
\leq\left\|\int_{\sigma}^{\sigma+\eta} D[G(\bar{x}(\tau), t)-G(x(\tau), t)]-(G(\bar{x}(\sigma), \sigma+\eta)-G(\bar{x}(\sigma), \sigma)-G(x(\sigma), \sigma+\eta)+G(x(\sigma), \sigma))\right\| \\
\leq\left\|G(\bar{x}(\sigma), \sigma+\eta)-G(\bar{x}(\sigma), \sigma)-\int_{\sigma}^{\sigma+\eta} D G(\bar{x}(\tau), t)\right\| \\
+\left\|G(x(\sigma), \sigma+\eta)-G(x(\sigma), \sigma)-\int_{\sigma}^{\sigma+\eta} D G(x(\tau), t)\right\| .
\end{gathered}
$$

Como

$\|G(\bar{x}(\sigma), \sigma+\eta)-G(\bar{x}(\sigma), \sigma)-G(x(\sigma), \sigma+\eta)+G(x(\sigma), \sigma)\| \leq\|\bar{x}(\sigma)-x(\sigma)\||h(\sigma+\eta)-h(\sigma)|=0$, uma vez que $\bar{x}(\sigma)=x(\sigma)$ e vale (1.9), segue por (2.6) e (2.7) que

$$
\left\|\int_{\sigma}^{\sigma+\eta} D[G(\bar{x}(\tau), t)-G(x(\tau), t)]\right\| \leq \frac{\eta \varepsilon}{K}
$$

Além disso, (2.2) implica

$$
\begin{gathered}
V(\sigma+\eta, \bar{x}(\sigma+\eta))-V(\sigma+\eta, x(\sigma+\eta)) \leq \\
\leq K\|\bar{x}(\sigma+\eta)-x(\sigma+\eta)\|=K\|\bar{x}(\sigma+\eta)-\bar{x}(\sigma)+x(\sigma)-x(\sigma+\eta)\| \\
=K\left\|\bar{x}(\sigma+\eta)-\bar{x}(\sigma)-\int_{\sigma}^{\sigma+\eta} D G(x(\tau), t)\right\| .
\end{gathered}
$$

Então (2.9), (2.5) e (2.8) implicam

$$
V(\sigma+\eta, \bar{x}(\sigma+\eta))-V(\sigma, \bar{x}(\sigma))
$$




$$
\begin{gathered}
=V(\sigma+\eta, \bar{x}(\sigma+\eta))-V(\sigma+\eta, x(\sigma+\eta))+V(\sigma+\eta, x(\sigma+\eta))-V(\sigma, x(\sigma)) \\
\leq K\left\|\bar{x}(\sigma+\eta)-\bar{x}(\sigma)-\int_{\sigma}^{\sigma+\eta} D G(x(\tau), t)\right\|+\eta \Phi(x(\sigma)) \\
\leq K\left\|\bar{x}(\sigma+\eta)-\bar{x}(\sigma)-\int_{\sigma}^{\sigma+\eta} D G(x(\tau), t)\right\|+\eta M \\
\leq K\left\|\bar{x}(\sigma+\eta)-\bar{x}(\sigma)-\int_{\sigma}^{\sigma+\eta} D G(\bar{x}(\tau), t)\right\| \\
+K\left\|\int_{\sigma}^{\sigma+\eta} D[G(\bar{x}(\tau), t)-G(x(\tau), t)]\right\|+\eta M \\
\leq K\left\|\bar{x}(\sigma+\eta)-\bar{x}(\sigma)-\int_{\sigma}^{\sigma+\eta} D G(\bar{x}(\tau), t)\right\|+\eta \varepsilon+\eta M .
\end{gathered}
$$

Dado $s \in[\gamma, v]$, defina

$$
P(s)=\bar{x}(s)-\int_{\gamma}^{s} D G(\bar{x}(\tau), t) .
$$

Como $\bar{x}$ é de variação limitada em $[\gamma, v]$, segue de (1.8) que $P$ também é de variação limitada $[\gamma, v]$. Então

$$
\begin{gathered}
\left\|\bar{x}(\sigma+\eta)-\bar{x}(\sigma)-\int_{\sigma}^{\sigma+\eta} D G(\bar{x}(\tau), t)\right\|=\|P(\sigma+\eta)-P(\sigma)\| \\
\leq \operatorname{var}_{\sigma}^{\sigma+\eta}(P)=\operatorname{var}_{\gamma}^{\sigma+\eta}(P)-\operatorname{var}_{\gamma}^{\sigma}(P) .
\end{gathered}
$$

Então, por (2.10), obtemos

$$
\begin{gathered}
V(\sigma+\eta, \bar{x}(\sigma+\eta))-V(\sigma, \bar{x}(\sigma)) \leq K\left(\operatorname{var}_{\gamma}^{\sigma+\eta}(P)-\operatorname{var}_{\gamma}^{\sigma}(P)\right)+\eta \varepsilon+\eta M \\
=f(\sigma+\eta)-f(\sigma)
\end{gathered}
$$

para $0 \leq \eta \leq \eta_{2}$, onde

$$
f(t)=K \operatorname{var}_{\gamma}^{t}(P)+\varepsilon t+M t .
$$

A função $f$ é de variação limitada em $[\gamma, v]$ e contínua à esquerda em $(\gamma, v]$. Por $(2.11)$ e pelo Lema 2.4, obtemos a desigualdade

$$
V(v, \bar{x}(v))-V(\gamma, \bar{x}(\gamma)) \leq K \operatorname{var}_{\gamma}^{v}(P)+\varepsilon(v-\gamma)+M(v-\gamma)
$$

Daí, como $\varepsilon>0$ pode ser tomado arbitrariamente pequeno, obtemos (2.4). 
O próximo resultado dará condições suficientes para que a solução nula de (2.1) seja variacionalmente estável.

Teorema 2.6 ([27], Teorema 10.13). Seja $V:\left[t_{0},+\infty\right) \times \overline{B_{\rho}} \rightarrow \mathbb{R}$ um funcional de Lyapunov, onde $\overline{B_{\rho}}=$ $\{y \in X:\|y\| \leq \rho\}, 0<\rho<c$. Suponha que $V$ satisfaça as seguintes condições adicionais:

(i) $V(t, 0)=0, t \in\left[t_{0},+\infty\right)$;

(ii) Existe uma constante $K>0$ tal que

$$
|V(t, z)-V(t, y)| \leq K\|z-y\|, \quad t \in\left[t_{0},+\infty\right), z, y \in \overline{B_{\rho}} .
$$

Então a solução trivial $x \equiv 0$ de (2.1) será variacionalmente estável.

Demonstração. Seja $\bar{x}:[\gamma, v] \rightarrow X$ uma função contínua à esquerda em $(\gamma, v]$ e de variação limitada em $[\gamma, v], \operatorname{com}[\gamma, v] \subset\left[t_{0},+\infty\right)$. Pela condição (iii) da Definição 2.3 e pelo Lema 2.5, temos

$$
V(t, \bar{x}(t)) \leq V(\gamma, \bar{x}(\gamma))+K \operatorname{var}_{\gamma}^{t}\left(\bar{x}(s)-\int_{\gamma}^{s} D G(\bar{x}(\tau), \bar{t})\right), \quad t \in[\gamma, v]
$$

Como $V$ é um funcional de Lyapunov, então existe uma função monótona crescente $b: \mathbb{R}_{+} \rightarrow \mathbb{R}_{+}$tal que $b(0)=0 \mathrm{e}$

$$
V(t, x) \geq b(\|x\|), \quad \text { para }(t, x) \in\left[t_{0},+\infty\right) \times \overline{B_{\rho}} .
$$

Dado $\varepsilon>0$, temos $b(\varepsilon)>0$. Seja $\delta(\varepsilon)>0$ tal que $2 K \delta(\varepsilon)<b(\varepsilon)$. Se $\|\bar{x}(\gamma)\|<\delta(\varepsilon)$ e

$$
\operatorname{var}_{\gamma}^{t}\left(\bar{x}(s)-\int_{\gamma}^{s} D G(\bar{x}(\tau), t)\right)<\delta(\varepsilon)
$$

então (2.12) implicará

$$
V(t, \bar{x}(t)) \leq 2 K \delta(\varepsilon)<b(\varepsilon), t \in[\gamma, v]
$$

já que $V(\gamma, \bar{x}(\gamma)) \leq|V(\gamma, \bar{x}(\gamma))| \leq K\|\bar{x}(\gamma)\|$ por $(i)$ e $(i i)$.

Por outro lado, se existir $u \in[\gamma, v]$ tal que $\|\bar{x}(u)\| \geq \varepsilon$, então (2.13) implicará

$$
V(u, \bar{x}(u)) \geq b(\|\bar{x}(u)\|) \geq b(\varepsilon)
$$

o que contradiz (2.14). Portanto $\|\bar{x}(t)\|<\varepsilon$ para todo $t \in[\gamma, v]$, donde segue o resultado. 
O próximo teorema mostra que, sob algumas condições, a solução nula da EDO generalizada (2.1) é variacionalmente assintoticamente estável.

Teorema 2.7 ([27], Teorema 10.14). Seja $V:\left[t_{0},+\infty\right) \times \overline{B_{\rho}} \rightarrow \mathbb{R}$ um funcional de Lyapunov, onde $\overline{B_{\rho}}=$ $\{y \in X:\|y\| \leq \rho\}, 0<\rho<c$. Suponha que V satisfaça a condições $(i)$ e (ii) do Teorema 2.6. Além disso, suponha que exista uma função contínua $\Phi: X \rightarrow \mathbb{R}$, satisfazendo $\Phi(0)=0$ e $\Phi(x)>0$ para $x \neq 0$, tal que para toda solução $x:[\gamma, v] \rightarrow B_{\rho}$ de (2.1), com $[\gamma, v] \subset\left[t_{0},+\infty\right)$, tenhamos

$$
\dot{V}(t, x(t)) \leq-\Phi(x(t)), \quad t \in[\gamma, v]
$$

Então a solução trivial $x \equiv 0$ de (2.1) será variacionalmente assintoticamente estável.

Demonstração. Note que a condição (2.15) implica $\dot{V}(t, x(t)) \leq 0$, para toda solução $x:[\gamma, v] \rightarrow B_{\rho}$ de (2.1). Portanto o Teorema 2.6 implica que a solução trivial $x \equiv 0$ de (2.1) é variacionalmente estável. Sendo assim, basta provar que a solução $x \equiv 0$ de (2.1) é variacionalmente atratora.

Como a solução trivial $x \equiv 0$ de (2.1) é variacionalmente estável, sabemos que:

(I) Existe $\delta_{0} \in(0, \rho)$ tal que se $\bar{x}:[\gamma, v] \rightarrow B_{\rho}$ for uma função de variação limitada em $[\gamma, v] \subset\left[t_{0},+\infty\right)$, contínua à esquerda em $(\gamma, v]$,

$$
\|\bar{x}(\gamma)\|<\delta_{0}
$$

$\mathrm{e}$

$$
\operatorname{var}_{\gamma}^{\nu}\left(\bar{x}(s)-\int_{\gamma}^{s} D G(\bar{x}(\tau), t)\right)<\delta_{0}
$$

então

$$
\|\bar{x}(t)\|<\rho, \quad t \in[\gamma, v] .
$$

(II) Para todo $\varepsilon>0$, existe $\delta=\delta(\varepsilon)>0, \delta<\varepsilon$ tal que se $\bar{x}:[\bar{\gamma}, \bar{v}] \rightarrow B_{\rho}$, [ $\left.\bar{\gamma}, \bar{v}\right]$ for uma função de variação limitada em $[\bar{\gamma}, \bar{v}] \subset\left[t_{0},+\infty\right)$, contínua à esquerda em $(\bar{\gamma}, \bar{v}]$,

$$
\|\bar{x}(\bar{\gamma})\|<\delta
$$

e

$$
\operatorname{var}_{\bar{\gamma}}^{\bar{v}}\left(\bar{x}(s)-\int_{\bar{\gamma}}^{s} D G(\bar{x}(\tau), t)\right)<\delta
$$

então

$$
\|\bar{x}(t)\|<\varepsilon, \quad t \in[\bar{\gamma}, \bar{v}] .
$$


$\operatorname{Sejam} \lambda(\varepsilon)=\min \left\{\delta_{0}, \delta\right\}<\varepsilon \mathrm{e}$

$$
N=\sup \{-\Phi(y): \lambda(\varepsilon) \leq\|y\| \leq \varepsilon\}=-\inf \{\Phi(y): \lambda(\varepsilon) \leq\|y\| \leq \varepsilon\}<0 .
$$

Definamos

$$
T(\varepsilon):=\min \left\{v-\gamma,-K \frac{\delta_{0}+\lambda(\varepsilon)}{N}\right\}>0 .
$$

Suponhamos que $\bar{x}:[\gamma, v] \rightarrow B_{\rho},[\gamma, v] \subset\left[t_{0},+\infty\right)$, seja uma função de variação limitada em $[\gamma, v]$, contínua à esquerda em $(\gamma, v]$ e tal que

$$
\|\bar{x}(\gamma)\|<\delta_{0}
$$

e

$$
\operatorname{var}_{\gamma}^{v}\left(\bar{x}(s)-\int_{\gamma}^{s} D G(\bar{x}(\tau), t)\right)<\lambda(\varepsilon)<\delta
$$

Queremos provar que

$$
\|\bar{x}(t)\|<\varepsilon, \quad t \in[\gamma, v] \cap[\gamma+T(\varepsilon),+\infty), \gamma \geq 0 .
$$

Afirmamos que existe um $t^{*} \in[\gamma, \gamma+T(\varepsilon)]$ tal que $\left\|\bar{x}\left(t^{*}\right)\right\|<\lambda(\varepsilon) \leq \delta$. Com efeito, suponhamos, por absurdo, que $\|\bar{x}(s)\| \geq \lambda(\varepsilon)$, para todo $s \in[\gamma, \gamma+T(\varepsilon)]$. Pelo Lema 2.5, pela condição (ii) do Teorema 2.6, por (2.18), (2.16) e (2.17), temos

$$
\begin{aligned}
V(\gamma+T(\varepsilon), \bar{x}(\gamma+T(\varepsilon))) & \leq V(\gamma, \bar{x}(\gamma))+K \operatorname{var}_{\gamma}^{\gamma+T(\varepsilon)}\left(\bar{x}(s)-\int_{\gamma}^{s} D G(\bar{x}(\tau), t)\right)+N T(\varepsilon) \\
& <K\|\bar{x}(\gamma)\|+K \lambda(\varepsilon)+N\left(-K \frac{\delta_{0}+\lambda(\varepsilon)}{N}\right) \\
& =K\|\bar{x}(\gamma)\|-K \delta_{0}<0 .
\end{aligned}
$$

Por outro lado, existe uma função monótona crescente $b: \mathbb{R}_{+} \rightarrow \mathbb{R}_{+}$tal que $b(0)=0$ e

$$
V(t, x) \geq b(\|x\|), \quad \text { para }(t, x) \in\left[t_{0},+\infty\right) \times \overline{B_{\rho}},
$$

uma vez que $V$ é um funcional de Lyapunov. Daí,

$$
V(\gamma+T(\varepsilon), \bar{x}(\gamma+T(\varepsilon))) \geq b(\|\bar{x}(\gamma+T(\varepsilon))\|) \geq b(\lambda(\varepsilon))>0
$$

Eis, pois, uma contradição. Por conseguinte, $\|\bar{x}(t)\| \leq \varepsilon$ para $t \in\left[t^{*}, v\right]$, uma vez que (II) vale para $\bar{\gamma}=t^{*}$ e $\bar{v}=v$. Além disso, $\|\bar{x}(t)\| \leq \varepsilon$ para $t>\gamma+T(\varepsilon)$, já que $t^{*} \in[\gamma, \gamma+T(\varepsilon)]$. Então a solução $x \equiv 0$ de (2.1) é variacionalmente atratora e a prova está finalizada. 


\subsection{Estabilidade exponencial variacional da solução trivial}

Assim como na seção anterior, vamos considerar $\Omega=B_{c} \times\left[t_{0},+\infty\right)$, onde $B_{c}=\{y \in X:\|y\|<c\}$, $\operatorname{com} c>0$. Consideraremos a EDO generalizada

$$
\frac{d x}{d \tau}=D G(x, t)
$$

onde $G \in \mathscr{F}(\Omega, h)$ e novamente $h:\left[t_{0},+\infty\right) \rightarrow \mathbb{R}$ é uma função não decrescente e contínua à esquerda. Também vamos supor que $G(0, t)-G(0, s)=0$, para $t, s \geq t_{0}$, a fim de que possamos garantir que $x \equiv 0$ seja uma solução da EDO generalizada (2.19) em $\left[t_{0},+\infty\right)$.

Em [3], introduzimos um conceito novo de estabilidade exponencial variacional para EDOs generalizadas que também foi baseado na variação das soluções de (2.19) em torno da solução nula $x \equiv 0$. Tal conceito segue abaixo.

Definição 2.8. Seja $\lambda$ uma constante positiva. A solução trivial $x \equiv 0$ de (2.19) será dita variacionalmente exponencialmente estável se, para todo $\varepsilon>0$, existir $\delta=\delta(\varepsilon)>0$ tal que se $\bar{x}:[\gamma, v] \rightarrow B_{c}$, $t_{0} \leq \gamma<v<+\infty$, for uma função de variação limitada em $[\gamma, v]$ e contínua à esquerda em $(\gamma, v]$ tal que

$$
\|\bar{x}(\gamma)\|<\delta
$$

$e$

$$
\operatorname{var}_{\gamma}^{\nu}\left(\bar{x}(s)-\int_{\gamma}^{s} D G(\bar{x}(\tau), t)\right)<\delta
$$

então

$$
\|\bar{x}(t)\|<\varepsilon \exp [-\lambda(t-\gamma)], \quad t \in[\gamma, v] .
$$

$\mathrm{Na}$ sequência, veremos sob quais condições podemos garantir que a solução trivial de (2.19) seja variacionalmente exponencialmente estável.

Teorema 2.9. Seja $V:\left[t_{0},+\infty\right) \times \overline{B_{\rho}} \rightarrow \mathbb{R}$ um funcional de Lyapunov onde $\overline{B_{\rho}}=\{y \in X:\|y\| \leq \rho\}$, $0<\rho<c$. Suponha que $V$ satisfaça as seguintes condições:

(i) $V(t, 0)=0, t \in\left[t_{0},+\infty\right)$;

(ii) Existe uma constante $K>0$ tal que

$$
|V(t, z)-V(t, y)| \leq K\|z-y\|, \quad t \in\left[t_{0},+\infty\right), z, y \in \overline{B_{\rho}}
$$


Então a solução trivial $x \equiv 0$ de (2.19) será variacionalmente exponencialmente estável.

Demonstração. Seja $\bar{x}:[\gamma, v] \rightarrow X$ uma função de variação limitada em $[\gamma, v]$ e contínua à esquerda em $(\gamma, v], \operatorname{com}[\gamma, v] \subset\left[t_{0},+\infty\right)$. Pela condição (iii) da Definição 2.3 e pelo Lema 2.5, temos

$$
V(t, \bar{x}(t)) \leq V(\gamma, \bar{x}(\gamma))+K \operatorname{var}_{\gamma}^{t}\left(\bar{x}(s)-\int_{\gamma}^{s} D G(\bar{x}(\tau), \bar{t})\right)
$$

para cada $t \in[\gamma, v]$.

Como $V$ é um funcional de Lyapunov, então existe uma função monótona crescente $b: \mathbb{R}_{+} \rightarrow \mathbb{R}_{+}$tal que $b(0)=0$ e

$$
V(t, x) \geq b(\|x\|), \quad \text { para }(t, x) \in\left[t_{0},+\infty\right) \times \overline{B_{\rho}} .
$$

Agora, fixemos $\lambda>0$ e seja $\varepsilon>0$ arbitrário. Pelas propriedades da função $b$, temos $b(\varepsilon \exp [-\lambda(v-$ $\gamma)])>0$.

Seja $\delta(\varepsilon)>0$ tal que $2 K \delta(\varepsilon)<b(\varepsilon \exp [-\lambda(v-\gamma)])$. Se $\|\bar{x}(\gamma)\|<\delta(\varepsilon)$ e

$$
\operatorname{var}_{\gamma}^{t}\left(\bar{x}(s)-\int_{\gamma}^{s} D G(\bar{x}(\tau), \bar{t})\right)<\delta(\varepsilon)
$$

então (2.20) implicará

$$
V(t, \bar{x}(t)) \leq 2 K \delta(\varepsilon)<b(\varepsilon \exp [-\lambda(v-\gamma)]), \quad t \in[\gamma, v],
$$

já que $V(\gamma, \bar{x}(\gamma)) \leq K\|\bar{x}(\gamma)\|$ por $(i)$ e $(i i)$.

Em contrapartida, se existir $u \in[\gamma, v]$ tal que $\|\bar{x}(u)\| \geq \varepsilon \exp [-\lambda(v-\gamma)]$, então (2.21) implicará

$$
V(u, \bar{x}(u)) \geq b(\|\bar{x}(u)\|) \geq b(\varepsilon \exp [-\lambda(v-\gamma)]),
$$

o que contradiz (2.22). Então $\|\bar{x}(t)\|<\varepsilon \exp [-\lambda(v-\gamma)]$ para todo $t \geq \gamma$. Mas note que

$$
\varepsilon \exp [-\lambda(v-\gamma)] \leq \varepsilon \exp [-\lambda(t-\gamma)]
$$

para todo $t \in[\gamma, v]$. Por conseguinte,

$$
\|\bar{x}(t)\|<\varepsilon \exp [-\lambda(t-\gamma)], \text { para } t \geq \gamma
$$


e o resultado segue.

\subsection{Limitação uniforme de soluções}

Esta seção será destinada a mostrar resultados sobre limitação de soluções de uma classe de EDOs generalizadas. Os resultados desta seção estão contidos no artigo [1].

No que segue, $\Omega=O \times\left[t_{0},+\infty\right)$, onde $O$ é um subconjunto aberto de $X$ e $t_{0}$ é um número real não negativo. Nós também suporemos que $G \in \mathscr{F}(\Omega, h)$, onde $h:\left[t_{0},+\infty\right) \rightarrow \mathbb{R}$ é uma função não decrescente e contínua à esquerda.

Consideremos a EDO generalizada

$$
\frac{d x}{d \tau}=D G(x, t)
$$

com condição inicial

$$
x\left(t_{0}\right)=z_{0},
$$

onde $t_{0} \geq 0$ e $z_{0} \in O$ é tal que $z_{0}+G\left(z_{0}, t_{0}+\right)-G\left(z_{0}, t_{0}\right) \in O$.

Seja $x(t)=x\left(t, t_{0}, z_{0}\right)$ a única solução de (2.23)-(2.24) definida num intervalo $J\left(t_{0}, z_{0}\right)=\left[t_{0}, \sigma\right)$.

Definição 2.10. A solução $x(t)=x\left(t, t_{0}, z_{0}\right)$ do sistema (2.23)-(2.24) será dita

i) Uniformemente limitada, se para todo $\alpha>0$, existir um $M=M(\alpha)>0$ tal que se

$$
\left\|z_{0}\right\|<\alpha
$$

então

$$
\|x(t)\|<M, \quad \text { para todo } t \geq t_{0} .
$$

ii) Quase uniformemente eventualmente limitada, se existir uma constante $B>0$ tal que para todo $\alpha>0$, existe uma constante $T=T(\alpha)>0$ tal que se

$$
\left\|z_{0}\right\|<\alpha
$$

então

$$
\|x(t)\|<B, \quad \text { para todo } t \geq t_{0}+T
$$


iii) Uniformemente eventualmente limitada, se for uniformemente limitada e quase uniformemente eventualmente limitada.

A seguir, usaremos funcionais de Lyapunov para obter resultados de limitação para a solução do problema de valor inicial (2.23)-(2.24).

O próximo teorema nos dará condições suficientes para que a única solução do sistema (2.23)-(2.24) seja uniformemente limitada.

Teorema 2.11 ([1], Teorema 4.3). Seja $V:\left[t_{0},+\infty\right) \times X \rightarrow \mathbb{R}$ um funcional de Lyapunov. Suponha que $V$ satisfaça as seguintes condições adicionais:

(i) $V(t, 0)=0$, para cada $t \in\left[t_{0},+\infty\right)$;

(ii) Para cada $a>0$, existe uma constante $K_{a}>0$ tal que

$$
|V(t, z)-V(t, y)| \leq K_{a}\|z-y\|, \quad t \in\left[t_{0},+\infty\right), \quad y, z \in B_{a},
$$

onde $B_{a}=\{x \in X:\|x\|<a\}$

(iii) A função $b: \mathbb{R}_{+} \rightarrow \mathbb{R}_{+}$da condição (ii) da Definição 2.3 é tal que $b(s) \rightarrow+\infty$ quando $s \rightarrow+\infty$.

Então a solução $x(t)=x\left(t, t_{0}, z_{0}\right)$ do problema de valor inicial (2.23)-(2.24) será uniformemente limitada.

Demonstração. Seja $x\left(t, t_{0}, z_{0}\right)$ a solução de (2.23)-(2.24) definida num intervalo $J\left(t_{0}, z_{0}\right)$. Suporemos que $J\left(t_{0}, z_{0}\right)$ seja o intervalo $\left[t_{0},+\infty\right)$, pois quando $J\left(t_{0}, z_{0}\right)=\left[t_{0}, \sigma\right)$, com $\sigma<+\infty$, a prova seguirá de forma análoga.

Afirmamos que $V(t, x(t)) \leq V\left(t_{0}, x\left(t_{0}\right)\right)$ para todo $t \geq t_{0}$. Com efeito, tomemos $t>t_{0}$ e $a=2 \sup _{s \in\left[t_{0}, t\right]}\|x(s)\|$. Sabemos que $x$ é contínua à esquerda em $\left(t_{0}, t\right]$ (veja o Lema 1.16) e de variação limitada em $\left[t_{0}, t\right]$ (veja o Corolário 1.17).

Pelo item (ii), existe $K_{a}>0$ tal que

$$
|V(\xi, z)-V(\xi, y)| \leq K_{a}\|z-y\|
$$


para quaisquer $\xi \in\left[t_{0}, t\right]$ e $z, y \in B_{a}$. Portanto, pelo Lema 2.5, temos

$$
V(t, x(t)) \leq V\left(t_{0}, x\left(t_{0}\right)\right)+K_{a} \operatorname{var}_{t_{0}}^{t}\left(x(s)-\int_{t_{0}}^{s} D G(x(\tau), t)\right) .
$$

Por outro lado, como $x$ é uma solução de (2.23), segue da Observação 2.2 que

$$
\operatorname{var}_{t_{0}}^{t}\left(x(s)-\int_{\gamma}^{s} D G(x(\tau), t)\right)=0
$$

Então $V(t, x(t)) \leq V\left(t_{0}, x\left(t_{0}\right)\right)$ e, como $t$ é arbitrário,

$$
V(t, x(t)) \leq V\left(t_{0}, x\left(t_{0}\right)\right), \quad \text { para todo } \quad t \geq t_{0} .
$$

Agora, seja $\alpha>0$ tal que

$$
\left\|x\left(t_{0}\right)\right\|<\alpha
$$

Como $z_{0}=x\left(t_{0}\right) \in B_{a}$, temos

$$
V\left(t_{0}, x\left(t_{0}\right)\right) \leq\left|V\left(t_{0}, x\left(t_{0}\right)\right)\right| \leq K_{a}\left\|x\left(t_{0}\right)\right\|,
$$

por $(i)$ e (ii). Além disso, como $b(s) \rightarrow+\infty$ quando $s \rightarrow+\infty$, podemos escolher um número positivo $M=M(\alpha)$ tal que

$$
K_{a} \alpha<b(M)
$$

Então, por (2.26), (2.28), (2.27) e (2.29), obtemos

$$
V(t, x(t))<b(M), \quad \text { para todo } t \geq t_{0}
$$

Para finalizar, precisamos provar que

$$
\|x(t)\|<M, \text { para } t \geq t_{0} .
$$

Suponhamos o contrário, ou seja, suponhamos que exista $t^{*} \geq t_{0}$ tal que $\left\|x\left(t^{*}\right)\right\| \geq M$. Então a condição (ii) da Definição 2.3 implica que

$$
V\left(t^{*}, x\left(t^{*}\right)\right) \geq b\left(\left\|x\left(t^{*}\right)\right\|\right) \geq b(M)
$$


o que contradiz (2.30). Portanto $\|x(t)\|<M$ para todo $t \geq t_{0}$ e o resultado segue.

O resultado seguinte estabelece condições para que a única solução do sistema (2.23)-(2.24) seja uniformemente eventualmente limitada.

Teorema 2.12 ([1], Teorema 4.4). Seja $V:\left[t_{0},+\infty\right) \times X \rightarrow \mathbb{R}$ um funcional de Lyapunov. Suponha que $V:\left[t_{0},+\infty\right) \times X \rightarrow \mathbb{R}$ satisfaça as condições $(i)$, (ii) e (iii) do Teorema 2.11. Suponha, ainda, que exista uma função contínua $\Phi: X \rightarrow \mathbb{R}$, com $\Phi(0)=0$ e $\Phi(x)>0$ para $x \neq 0$, tal que para toda solução $x:[\gamma, v] \rightarrow X$ de (2.23), onde $[\gamma, v] \subset\left[t_{0},+\infty\right)$, tenhamos

$$
\dot{V}(t, x(t)) \leq-\Phi(x(t)), \quad t \in[\gamma, v] .
$$

Então a solução $x(t)=x\left(t, t_{0}, z_{0}\right)$ do problema de valor inicial (2.23)-(2.24) será uniformemente eventualmente limitada.

Demonstração. Note que a condição (2.31) implica $\dot{V}(t, x(t)) \leq 0$, para toda solução $x:[\gamma, v] \rightarrow X$ de (2.1). Então, o Teorema 2.11 implica que a solução $x(t)=x\left(t, t_{0}, z_{0}\right)$ de (2.23)-(2.24) definida no intervalo $J\left(t_{0}, z_{0}\right)=\left[t_{0},+\infty\right)$ é uniformemente limitada. Portanto basta provar que $x\left(t, t_{0}, z_{0}\right)$ é quase uniformemente eventualmente limitada.

Como a solução $x(t)$ de (2.23)-(2.24) é uniformemente limitada, dado $\alpha>0$, existe um número positivo $M=M(\alpha)$ tal que se

$$
\left\|z_{0}\right\|<\alpha
$$

então

$$
\|x(t)\|<M, \text { para todo } t \geq t_{0}
$$

Seja $[\bar{t},+\infty) \subset\left[t_{0},+\infty\right)$ e definamos $y:[\bar{t},+\infty) \rightarrow X$ por $y(t)=x(t)$ para todo $t \in[\bar{t},+\infty)$. Note que se $\|y(\bar{t})\|<\rho$, onde $\rho>0$, então existirá $B>\rho$ tal que

$$
\|y(t)\|<B, \text { para todo } t \geq \bar{t},
$$

já que $x(t)$ é uniformemente limitada.

Sejam $\rho, \alpha$ e $B$ como acima e tomemos $\lambda=\min \{\alpha, \rho\}$. Seja $a=2\left\|x\left(t_{0}\right)\right\|$ e definamos

$$
N:=\sup \{-\Phi(w) ; \lambda \leq\|w\| \leq B\}<0
$$


$\mathrm{e}$

$$
T(\alpha):=\frac{-2 K_{a} \alpha}{N}>0
$$

Vamos provar que $\|x(t)\|<B$, para todo $t \geq t_{0}+T(\alpha)$. Afirmamos que existe $t^{*} \in\left[t_{0}+\frac{T(\alpha)}{2}, t_{0}+T(\alpha)\right]$ tal que $\left\|x\left(t^{*}\right)\right\|<\lambda$. De fato, suponhamos, por absurdo, que $\|x(s)\| \geq \lambda$, para todo $s \in\left[t_{0}+\frac{T(\alpha)}{2}, t_{0}+T(\alpha)\right]$. Tomando $\bar{a}=2 \sup \left\{\|x(s)\|: s \in\left[t_{0}+\frac{T(\alpha)}{2}, t_{0}+T(\alpha)\right]\right\}$, pela condição (ii) do Teorema 2.11, existe $K_{\bar{a}}>0$ tal que

$$
|V(t, y)-V(t, z)| \leq K_{\bar{a}}\|z-y\|
$$

para quaisquer $t \in\left[t_{0}+\frac{T(\alpha)}{2}, t_{0}+T(\alpha)\right]$ e $z, y \in B_{\bar{a}}$. Então, pelo Lema 2.5, por (2.25), pelas condições (i) e (ii) do Teorema 2.11 e também por (2.32) e (2.35), temos

$$
\begin{aligned}
& \qquad V\left(t_{0}+T(\alpha), x\left(t_{0}+T(\alpha)\right)\right) \leq \\
& \leq V\left(t_{0}+\frac{T(\alpha)}{2}, x\left(t_{0}+\frac{T(\alpha)}{2}\right)\right)+K_{\bar{a}} \operatorname{var}_{t_{0}+\frac{T(\alpha)}{2}}^{t_{0}+T(\alpha)}\left(x(s)-\int_{t_{0}+\frac{T(\alpha)}{2}}^{s} D G(x(\tau), t)\right)+ \\
& +\frac{T(\alpha)}{2} \sup \left\{-\Phi(x(t)): t_{0}+\frac{T(\alpha)}{2} \leq t \leq t_{0}+T(\alpha)\right\} \\
& \leq V\left(t_{0}, x\left(t_{0}\right)\right)+\frac{T(\alpha)}{2} \sup \{-\Phi(w): \lambda \leq\|w\| \leq B\} \\
& \leq K_{a}\left\|x\left(t_{0}\right)\right\|-K_{a} \alpha<K_{a} \alpha-K_{a} \alpha=0 .
\end{aligned}
$$

É importante mencionar que, a prova de que a desigualdade $V\left(t_{0}+\frac{T(\alpha)}{2}, x\left(t_{0}+\frac{T(\alpha)}{2}\right)\right) \leq V\left(t_{0}, x\left(t_{0}\right)\right)$ vale, segue como em (2.26) na prova do teorema anterior.

Por outro lado, pela condição (ii) da Definição 2.3 , temos

$$
V\left(t_{0}+T(\alpha), x\left(t_{0}+T(\alpha)\right)\right) \geq b\left(\left\|x\left(t_{0}+T(\alpha)\right)\right\|\right) \geq b(\lambda)>0
$$

o que contradiz (2.36). Por conseguinte, $\|x(t)\|<B$ para $t \geq t^{*}$, visto que (2.34) vale para $\bar{t}=t^{*}$. Além disso, $\|x(t)\|<B$ para $t>t_{0}+T(\alpha)$, uma vez que $t^{*} \in\left[t_{0}+\frac{T(\alpha)}{2}, t_{0}+T(\alpha)\right]$. Portanto a solução $x(t)=$ $x\left(t, t_{0}, z_{0}\right)$ de (2.23)-(2.24) é quase uniformemente eventualmente limitada e a prova está completa. 


\section{EDFRs com impulsos em tempo variável e EDOs generalizadas}

Neste capítulo, descreveremos uma determinada classe de EDFRs com impulsos em tempo variável. Posteriormente, mostraremos que esta classe de EDFRs com impulsos em tempo variável poderá ser identificada, de maneira biunívoca, com uma certa classe de EDOs generalizadas. Obteremos, então, uma correspondência entre as soluções de tais classes de equações diferenciais. Observamos que essa correspondência será fundamental para a construção dos nossos resultados.

As principais referências para este capítulo são [8] e [10].

\subsection{Descrição da classe de EDFRs impulsivas}

Seja $\mathbb{R}^{n}$ o espaço euclideano $\mathrm{n}$-dimensional com norma $|\cdot|$.

Denotaremos por $G^{-}\left([a, b], \mathbb{R}^{n}\right)$ o espaço de Banach das funções de $[a, b] \subset \mathbb{R}$ em $\mathbb{R}^{n}$ que são regradas e contínuas à esquerda, munido da norma usual do supremo, dada por $\|f\|=\sup _{s \in[a, b]}|f(s)|$, para $f \in$ $G^{-}\left([a, b], \mathbb{R}^{n}\right)$.

Denotaremos por $G^{-}\left([a,+\infty), \mathbb{R}^{n}\right)$ o espaço das funções $\psi:[a,+\infty) \rightarrow \mathbb{R}^{n}$ tais que para todo $b>a$, a restrição $\left.\psi\right|_{[a, b]} \in G^{-}\left([a, b], \mathbb{R}^{n}\right)$. Em $G^{-}\left([a,+\infty), \mathbb{R}^{n}\right)$, consideraremos a topologia da convergência localmente uniforme.

Sejam $r>0$ e $t_{0} \geq 0$. Dada uma função $y \in G^{-}\left(\left[t_{0}-r,+\infty\right), \mathbb{R}^{n}\right)$, e $t \in\left[t_{0},+\infty\right)$, definiremos $y_{t} \in$ $G^{-}\left([-r, 0], \mathbb{R}^{n}\right)$ por

$$
y_{t}(\theta)=y(t+\theta), \quad \theta \in[-r, 0] .
$$


Consideraremos a EDFR com ação impulsiva

$$
\begin{cases}\dot{y}(t)=f\left(y_{t}, t\right), & t \neq \tau_{k}(y(t)), \quad t \geq t_{0} \\ \Delta y(t)=I_{k}(y(t)), & t=\tau_{k}(y(t)), \quad k=0,1,2, \ldots\end{cases}
$$

com condição inicial

$$
y_{t_{0}}=\phi
$$

onde $\phi \in G^{-}\left([-r, 0], \mathbb{R}^{n}\right)$ e $f: G^{-}\left([-r, 0], \mathbb{R}^{n}\right) \times\left[t_{0},+\infty\right) \rightarrow \mathbb{R}^{n}$. Vamos considerar, também, que os operadores de impulso $I_{k}(x), k=0,1,2, \ldots$, sejam funções contínuas de $\mathbb{R}^{n}$ em $\mathbb{R}^{n}$ e que

$$
\Delta y(t)=y(t+)-y(t-)=y(t+)-y(t)
$$

para quaisquer $y \in G^{-}\left(\left[t_{0}-r,+\infty\right), \mathbb{R}^{n}\right)$ e $t \geq t_{0}$.

Suporemos que $\tau_{0}(x) \equiv t_{0}$, para todo $x \in \mathbb{R}^{n} \mathrm{e}$, para cada $k=1,2, \ldots$, definiremos o conjunto

$$
S_{k}=\left\{(t, x) \in\left[t_{0},+\infty\right) \times \mathbb{R}^{n}: t=\tau_{k}(x)\right\}
$$

Denotaremos por $m\left(\tau_{k}\right)$ o número de vezes que as curvas integrais do sistema (3.1)-(3.2) encontram as hipersuperfícies $S_{k}, k=0,1,2, \ldots$ Por $t_{k}^{i}$ denotaremos o i-ésimo momento em que as curvas integrais do sistema (3.1)-(3.2) encontram as hipersuperfícies $S_{k}$, com $i=1, \ldots, m\left(\tau_{k}\right)$ e $k=0,1,2, \ldots$.

Note que, se $t_{0}$ for um momento de impulso do sistema (3.1)-(3.2), então $m\left(\tau_{0}\right)=1$, já que $\tau_{0} \equiv t_{0}$. Neste caso, vamos considerar $t_{0}=t_{0}^{1}$.

Em relação às superfícies $\tau_{k}, k=1,2, \ldots$, vamos considerar as seguintes condições:

(C1) $\tau_{k} \in C\left(\mathbb{R}^{n},\left(t_{0},+\infty\right)\right), k=1,2, \ldots$, onde $C\left(\mathbb{R}^{n},\left(t_{0},+\infty\right)\right)$ é o espaço de Banach das funções contínuas de $\mathbb{R}^{n}$ em $\left(t_{0},+\infty\right)$, com a norma usual do supremo;

(C2) $t_{0}<\tau_{1}(x)<\tau_{2}(x)<\ldots$, para cada $x \in \mathbb{R}^{n}$;

(C3) $\tau_{k}(x) \rightarrow+\infty$ quando $k \rightarrow+\infty$ uniformemente em $x \in \mathbb{R}^{n}$;

(C4) As curvas integrais do sistema (3.1)-(3.2) encontram sucessivamente as hipersuperfícies $S_{1}, S_{2}, \ldots$ um número finito de vezes;

(C5) $t_{k}^{i}<t_{k}^{i+1}, i=1, \ldots, m\left(\tau_{k}\right)-1$, para todo $k=1,2, \ldots$ 
Observação 3.1. Neste trabalho, o momento inicial t $t_{0}$ pode ser considerado como um momento em que o sistema (3.1)-(3.2) sofre impulso ou não. Consideraremos $I_{0} \equiv 0$, se $t_{0}$ não for um momento de impulso.

Seja $P C_{1} \subset G^{-}\left(\left[t_{0}-r,+\infty\right), \mathbb{R}^{n}\right)$ um conjunto aberto (na topologia da convergência localmente uniforme em $\left.G^{-}\left(\left[t_{0}-r,+\infty\right), \mathbb{R}^{n}\right)\right)$ com a seguinte propriedade: se $y$ é um elemento de $P C_{1}$ e $\bar{t} \in\left[t_{0},+\infty\right)$, então $\bar{y}$ dada por

$$
\bar{y}(t)=\left\{\begin{array}{l}
y(t), t_{0}-r \leq t \leq \bar{t}, \\
y(\bar{t}), \bar{t}<t<+\infty,
\end{array}\right.
$$

também é um elemento de $P C_{1}$. Em particular, qualquer bola aberta em $G^{-}\left(\left[t_{0}-r,+\infty\right), \mathbb{R}^{n}\right)$ tem essa propriedade.

Vamos supor, também, que $f: G^{-}\left([-r, 0], \mathbb{R}^{n}\right) \times\left[t_{0},+\infty\right) \rightarrow \mathbb{R}^{n}$ seja tal que para cada $y \in G^{-}\left(\left[t_{0}-\right.\right.$ $\left.r,+\infty), \mathbb{R}^{n}\right), t \mapsto f\left(y_{t}, t\right)$ é localmente Lebesgue integrável em $t \in\left[t_{0},+\infty\right)$ e, além disso:

(A) Existe uma função localmente Lebesgue integrável $M:\left[t_{0},+\infty\right) \rightarrow \mathbb{R}$ tal que para todo $x \in P C_{1}$ e para quaisquer $u_{1}, u_{2} \in\left[t_{0},+\infty\right)$,

$$
\left|\int_{u_{1}}^{u_{2}} f\left(x_{s}, s\right) d s\right| \leq \int_{u_{1}}^{u_{2}} M(s) d s
$$

(B) Existe uma função localmente Lebesgue integrável $L:\left[t_{0},+\infty\right) \rightarrow \mathbb{R}$ tal que para quaisquer $x, y \in$ $P C_{1}$ e $u_{1}, u_{2} \in\left[t_{0},+\infty\right)$,

$$
\left|\int_{u_{1}}^{u_{2}}\left[f\left(x_{s}, s\right)-f\left(y_{s}, s\right)\right] d s\right| \leq \int_{u_{1}}^{u_{2}} L(s)\left\|x_{s}-y_{s}\right\| d s .
$$

Para os operadores de impulso $I_{k}: \mathbb{R}^{n} \rightarrow \mathbb{R}^{n}, k=0,1,2, \ldots$, vamos supor as seguintes condições:

(A') Existe uma constante $K_{1}>0$ tal que para todo $k=0,1,2, \ldots$ e para todo $x \in \mathbb{R}^{n}$,

$$
\left|I_{k}(x)\right| \leq K_{1}
$$

(B') Existe uma constante $K_{2}>0$ tal que para todo $k=0,1,2, \ldots$ e para quaisquer $x, y \in \mathbb{R}^{n}$,

$$
\left|I_{k}(x)-I_{k}(y)\right| \leq K_{2}|x-y| .
$$

Vejamos a definição de uma solução local do problema de valor inicial (3.1)-(3.2). 
Definição 3.2. Seja $\sigma>0$. Considere o sistema (3.1)-(3.2), onde $f: G^{-}\left([-r, 0], \mathbb{R}^{n}\right) \times\left[t_{0}, t_{0}+\sigma\right] \rightarrow \mathbb{R}^{n}$ é tal que, para todo $y \in G^{-}\left(\left[t_{0}-r, t_{0}+\sigma\right], \mathbb{R}^{n}\right), t \mapsto f\left(y_{t}, t\right)$ é Lebesgue integrável em $t \in\left[t_{0}, t_{0}+\sigma\right]$. Se existir uma função $y \in G^{-}\left(\left[t_{0}-r, t_{0}+\sigma\right], \mathbb{R}^{n}\right)$ tal que

(i) $\dot{y}(t)=f\left(y_{t}, t\right)$, para quase todo $t \in\left[t_{0}, t_{0}+\sigma\right] \backslash\left\{s \in\left[t_{0}, t_{0}+\sigma\right]: s=\tau_{k}(y(s)), k=0,1,2, \ldots\right\}$;

(ii) $y(t+)=y(t)+I_{k}(y(t)), t=\tau_{k}(y(t)) \in\left[t_{0}, t_{0}+\sigma\right], \quad k=0,1,2, \ldots ;$

(iii) $y_{t_{0}}=\phi$,

então y será dita uma solução de (3.1) em $\left[t_{0}-r, t_{0}+\sigma\right]$ com condição inicial $\left(\phi, t_{0}\right)$.

Denotamos por $y(t)=y\left(t, t_{0}, \phi\right)$ uma solução local do problema de valor inicial (3.1)-(3.2). O próximo resultado nos fornece uma formulação integral da solução $y(t)=y\left(t, t_{0}, \phi\right)$ de (3.1)-(3.2).

Lema 3.3. Considere o problema de valor inicial (3.1)-(3.2) e suponha que a função $f: G^{-}\left([-r, 0], \mathbb{R}^{n}\right) \times$ $\left[t_{0}, t_{0}+\sigma\right] \rightarrow \mathbb{R}^{n}$ seja tal que, para todo $y \in G^{-}\left(\left[t_{0}-r, t_{0}+\sigma\right], \mathbb{R}^{n}\right), t \mapsto f\left(y_{t}, t\right)$ é Lebesgue integrável em $t \in\left[t_{0}, t_{0}+\sigma\right]$. Então $y \in G^{-}\left(\left[t_{0}-r, t_{0}+\sigma\right], \mathbb{R}^{n}\right)$ é uma solução de (3.1)-(3.2) em $\left[t_{0}-r, t_{0}+\sigma\right] \mathrm{se}, e$ somente se,

$$
y(t)=\left\{\begin{array}{l}
\phi\left(t-t_{0}\right), \quad t \in\left[t_{0}-r, t_{0}\right], \\
\phi(0)+\int_{t_{0}}^{t} f\left(y_{s}, s\right) d s+\sum_{\substack{t_{0}<t_{k}^{i}<t, i=1, \ldots, m\left(\tau_{k}\right)}} I_{k}\left(y\left(t_{k}^{i}\right)\right), \quad t \in\left[t_{0}, t_{0}+\sigma\right], k=1, \ldots, j,
\end{array}\right.
$$

se to não for um momento de impulso do sitema (3.1)-(3.2) e

$$
y(t)=\left\{\begin{array}{l}
\phi\left(t-t_{0}\right), \quad t \in\left[t_{0}-r, t_{0}\right], \\
\phi(0)+I_{0}(\phi(0))+\int_{t_{0}}^{t} f\left(y_{s}, s\right) d s+\sum_{\substack{t_{0}<t_{k}^{i}<t, i=1, \ldots, m\left(\tau_{k}\right)}} I_{k}\left(y\left(t_{k}^{i}\right)\right), \quad t \in\left(t_{0}, t_{0}+\sigma\right], k=1, \ldots, j,
\end{array}\right.
$$

caso to seja um momento de impulso do sistema (3.1)-(3.2), onde jé tal que $t_{0}<\tau_{1}(x)<\tau_{2}(x)<\ldots<$ $\tau_{j}(x) \leq t_{0}+\sigma$ 


\subsection{Correspondência entre EDFRs impulsivas e EDOs generalizadas}

Sejam $t_{0} \geq 0, \sigma>0$ e $r>0$. Para $y \in P C_{1}$ e $t \in\left[t_{0}, t_{0}+\sigma\right]$, definamos

$$
F(y, t)(\vartheta)=\left\{\begin{array}{l}
0, t_{0}-r \leq \vartheta \leq t_{0} \text { ou } t_{0}-r \leq t \leq t_{0} \\
\int_{t_{0}}^{\vartheta} f\left(y_{s}, s\right) d s, t_{0} \leq \vartheta \leq t \leq t_{0}+\sigma \\
\int_{t_{0}}^{t} f\left(y_{s}, s\right) d s, t_{0} \leq t \leq \vartheta \leq t_{0}+\sigma
\end{array}\right.
$$

Note que, para cada par $(y, t) \in P C_{1} \times\left[t_{0}, t_{0}+\sigma\right]$, a equação (3.3) define um elemento $F(y, t)$ de $C\left(\left[t_{0}-r, t_{0}+\sigma\right], \mathbb{R}^{n}\right)$, ou seja,

$$
F: P C_{1} \times\left[t_{0}, t_{0}+\sigma\right] \rightarrow C\left(\left[t_{0}-r, t_{0}+\sigma\right], \mathbb{R}^{n}\right)
$$

e $F(y, t)(\tau) \in \mathbb{R}^{n}$ é o valor de $F(y, t)$ em um ponto $\tau \in\left[t_{0}-r, t_{0}+\sigma\right]$, onde $C\left([a, b], \mathbb{R}^{n}\right)$ denota o espaço de Banach das funções contínuas que aplicam $[a, b]$ em $\mathbb{R}^{n}$, munido da norma usual do supremo.

Suponhamos que as condições $(A)$ e $(B)$ da seção anterior estejam satisfeitas para a aplicação $f(\phi, t)$ : $G^{-}\left([-r, 0], \mathbb{R}^{n}\right) \times\left[t_{0}, t_{0}+\sigma\right] \rightarrow \mathbb{R}^{n}$

Dados $x \in P C_{1}$ e $t_{0} \leq s_{1}<s_{2}<t_{0}+\sigma$, para $F: P C_{1} \times\left[t_{0}, t_{0}+\sigma\right] \rightarrow C\left(\left[t_{0}-r, t_{0}+\sigma\right], \mathbb{R}^{n}\right)$ dada por (3.3), temos

$$
F\left(x, s_{2}\right)(\vartheta)-F\left(x, s_{1}\right)(\vartheta)=\left\{\begin{array}{l}
0, \vartheta \in\left[t_{0}-r, s_{1}\right], \\
\int_{s_{1}}^{\vartheta} f\left(x_{s}, s\right) d s, \vartheta \in\left[s_{1}, s_{2}\right], \\
\int_{s_{1}}^{s_{2}} f\left(x_{s}, s\right) d s, \vartheta \in\left[s_{2}, t_{0}+\sigma\right] .
\end{array}\right.
$$

Portanto, para $x \in P C_{1}$ e para $t_{0} \leq s_{1}<s_{2}<t_{0}+\sigma$, a condição $(A)$ implica que

$$
\begin{gathered}
\left\|F\left(x, s_{2}\right)-F\left(x, s_{1}\right)\right\|=\sup _{\vartheta \in\left[t_{0}-r, t_{0}+\sigma\right]}\left|F\left(x, s_{2}\right)(\vartheta)-F\left(x, s_{1}\right)(\vartheta)\right|= \\
=\sup _{\vartheta \in\left[s_{1}, s_{2}\right]}\left|F\left(x, s_{2}\right)(\vartheta)-F\left(x, s_{1}\right)(\vartheta)\right|=\sup _{\vartheta \in\left[s_{1}, s_{2}\right]}\left|\int_{s_{1}}^{\vartheta} f\left(x_{s}, s\right) d s\right| \leq \\
\leq \sup _{\vartheta \in\left[s_{1}, s_{2}\right]} \int_{s_{1}}^{\vartheta} M(s) d s=\int_{s_{1}}^{s_{2}} M(s) d s .
\end{gathered}
$$


Analogamente, se $x, y \in P C_{1}$ e $t_{0} \leq s_{1}<s_{2}<t_{0}+\sigma$, por (3.4) e pela condição $(B)$, obtemos

$$
\begin{gathered}
\left\|F\left(x, s_{2}\right)-F\left(x, s_{1}\right)-F\left(y, s_{2}\right)+F\left(y, s_{1}\right)\right\|= \\
=\sup _{\vartheta \in\left[s_{1}, s_{2}\right]}\left|\int_{s_{1}}^{\vartheta}\left[f\left(x_{s}, s\right)-f\left(y_{s}, s\right) d s\right]\right| \leq \int_{s_{1}}^{s_{2}} L(s)\left\|x_{s}-y_{s}\right\| d s \leq \\
\leq \sup _{\vartheta \in\left[s_{1}-r, s_{2}\right]}|x(\vartheta)-y(\vartheta)| \int_{s_{1}}^{s_{2}} L(s) d s \leq\|x-y\| \int_{s_{1}}^{s_{2}} L(s) d s .
\end{gathered}
$$

Definamos $h_{1}:\left[t_{0}, t_{0}+\sigma\right] \rightarrow \mathbb{R}$ pondo

$$
h_{1}(t)=\int_{t_{0}}^{t}[M(s)+L(s)] d s, t \in\left[t_{0}, t_{0}+\sigma\right] .
$$

Então a função $h_{1}$ é (absolutamente) contínua e não decrescente, pois $M, L:\left[t_{0}, t_{0}+\sigma\right] \rightarrow \mathbb{R}$ são funções não negativas e Lebesgue integráveis.

Portanto, pelas relações (3.5) e (3.6), temos

$$
\left\|F\left(x, s_{2}\right)-F\left(x, s_{1}\right)\right\| \leq\left|h_{1}\left(s_{2}\right)-h_{1}\left(s_{1}\right)\right|
$$

para quaisquer $\left(x, s_{2}\right),\left(x, s_{1}\right) \in P C_{1} \times\left[t_{0}, t_{0}+\sigma\right] \mathrm{e}$

$$
\left\|F\left(x, s_{2}\right)-F\left(x, s_{1}\right)-F\left(y, s_{2}\right)+F\left(y, s_{1}\right)\right\| \leq\|x-y\|\left|h_{1}\left(s_{2}\right)-h_{1}\left(s_{1}\right)\right|
$$

para quaisquer $\left(x, s_{2}\right),\left(x, s_{1}\right),\left(y, s_{2}\right),\left(y, s_{1}\right) \in P C_{1} \times\left[t_{0}, t_{0}+\sigma\right]$. Por conseguinte, $F \in \mathscr{F}\left(\Omega, h_{1}\right)$, onde $\Omega=P C_{1} \times\left[t_{0}, t_{0}+\sigma\right]$.

Agora, vamos supor que as condições $(C 1)$ até $(C 5)$ estejam satisfeitas. Para $y \in P C_{1}$ e $t \in\left[t_{0}, t_{0}+\sigma\right]$, definamos

$$
J(y, t)(\vartheta)=\sum_{k=0}^{j} \sum_{i=1}^{m\left(\tau_{k}\right)} H_{k}^{i}(t) H_{k}^{i}(\vartheta) I_{k}\left(y\left(t_{k}^{i}\right)\right)
$$

onde $\vartheta \in\left[t_{0}-r,+\infty\right), j$ é tal que $t_{0} \equiv \tau_{0}(x)<\tau_{1}(x)<\tau_{2}(x)<\ldots<\tau_{j}(x) \leq t_{0}+\sigma$ e $H_{k}^{i}$ denota a função de Heaviside contínua à esquerda concentrada em $t_{k}^{i}$, isto é ,

$$
H_{k}^{i}(t)=\left\{\begin{array}{l}
0, \text { para } t_{0} \leq t \leq t_{k}^{i}, \\
1, \text { para } t>t_{k}^{i} .
\end{array}\right.
$$


Vamos considerar que as condições $\left(A^{\prime}\right)$ e $\left(B^{\prime}\right)$ do capítulo anterior estejam satisfeitas. Então, para $y \in P C_{1}$ e $t_{0} \leq s_{1} \leq s_{2} \leq t_{0}+\sigma$, temos

$$
J\left(y, s_{2}\right)(\vartheta)-J\left(y, s_{1}\right)(\vartheta)=\sum_{k=0}^{j} \sum_{i=1}^{m\left(\tau_{k}\right)}\left[H_{k}^{i}\left(s_{2}\right)-H_{k}^{i}\left(s_{1}\right)\right] H_{k}^{i}(\vartheta) I_{k}\left(y\left(t_{k}^{i}\right)\right)
$$

onde $\vartheta \in\left[t_{0}-r, t_{0}+\sigma\right]$. Logo, para $t_{0} \leq s_{1}<s_{2} \leq t_{0}+\sigma$ e $y \in P C_{1}$, temos

$$
\begin{aligned}
\left\|J\left(x, s_{2}\right)-J\left(x, s_{1}\right)\right\| \leq & \sup _{\vartheta \in\left[t_{0}-r, t_{0}+\sigma\right]} \sum_{k=0}^{j} \sum_{i=1}^{m\left(\tau_{k}\right)}\left[H_{k}^{i}\left(s_{2}\right)-H_{k}^{i}\left(s_{1}\right)\right] H_{k}^{i}(\vartheta)\left|I_{k}\left(y\left(t_{k}^{i}\right)\right)\right| \\
& \leq K_{1} \sum_{k=0}^{j} \sum_{i=1}^{m\left(\tau_{k}\right)}\left[H_{k}^{i}\left(s_{2}\right)-H_{k}^{i}\left(s_{1}\right)\right]
\end{aligned}
$$

e, analogamente, se $x, y \in P C_{1}$ e $t_{0} \leq s_{1} \leq s_{2} \leq t_{0}+\sigma$, concluímos que

$$
\left\|J\left(x, s_{2}\right)-J\left(x, s_{1}\right)-J\left(y, s_{2}\right)+J\left(x, s_{1}\right)\right\| \leq \sum_{k=0}^{j} \sum_{i=1}^{m\left(\tau_{k}\right)} K_{2}\left[H_{k}^{i}\left(s_{2}\right)-H_{k}^{i}\left(s_{1}\right)\right]\|x-y\|
$$

Definamos $h_{2}:\left[t_{0}, t_{0}+\sigma\right] \rightarrow \mathbb{R}$ pondo

$$
h_{2}(t)=\max \left(K_{1}, K_{2}\right) \sum_{k=0}^{j} \sum_{i=1}^{m\left(\tau_{k}\right)} H_{k}^{i}(t)
$$

Então $h_{2}$ é contínua à esquerda e não decrescente. Além disso, por (3.12) e (3.13), temos

$$
\left\|J\left(x, s_{2}\right)-J\left(x, s_{1}\right)\right\| \leq h_{2}\left(s_{2}\right)-h_{2}\left(s_{1}\right)
$$

$\mathrm{e}$

$$
\left\|J\left(x, s_{2}\right)-J\left(x, s_{1}\right)-J\left(y, s_{2}\right)+J\left(x, s_{1}\right)\right\| \leq\|x-y\|\left[h_{2}\left(s_{2}\right)-h_{2}\left(s_{1}\right)\right]
$$

sempre que $x, y \in P C_{1}$ e $t_{0} \leq s_{1}<s_{2} \leq t_{0}+\sigma$. Logo $J \in \mathscr{F}\left(\Omega, h_{2}\right)$, onde $\Omega=P C_{1} \times\left[t_{0}, t_{0}+\sigma\right]$.

Tomando $F(y, t)$ dada por (3.3) e $J(y, t)$ dada por (3.10), definamos

$$
G(y, t)(\vartheta)=F(y, t)(\vartheta)+J(y, t)(\vartheta)
$$

para $y \in P C_{1}, t \in\left[t_{0}, t_{0}+\sigma\right]$ e $\vartheta \in\left[t_{0}-r, t_{0}+\sigma\right]$. Claramente o valor de $G(y, t)$ pertence a $G^{-}\left(\left[t_{0}-r, t_{0}+\right.\right.$ 
$\left.\sigma], \mathbb{R}^{n}\right)$, ou seja, temos uma função

$$
G: P C_{1} \times\left[t_{0}, t_{0}+\sigma\right] \rightarrow G^{-}\left(\left[t_{0}-r, t_{0}+\sigma\right], \mathbb{R}^{n}\right)
$$

Além disso, pelas desigualdades (3.8) e (3.15), temos

$$
\begin{aligned}
\left\|G\left(x, s_{2}\right)-G\left(x, s_{1}\right)\right\| & \leq\left\|F\left(x, s_{2}\right)-F\left(x, s_{1}\right)\right\|+\left\|J\left(x, s_{2}\right)-J\left(x, s_{1}\right)\right\| \leq \\
& \leq h_{1}\left(s_{2}\right)-h_{1}\left(s_{1}\right)+h_{2}\left(s_{2}\right)-h_{2}\left(s_{1}\right)=h\left(s_{2}\right)-h\left(s_{1}\right),
\end{aligned}
$$

onde $h(t)=h_{1}(t)+h_{2}(t)$ é não decrescente e contínua à esquerda, $x \in P C_{1}$ e $t_{0} \leq s_{1}<s_{2} \leq t_{0}+\sigma$.

Analogamente, as desigualdades (3.9) e (3.16) implicam

$$
\left\|G\left(x, s_{2}\right)-G\left(x, s_{1}\right)-G\left(y, s_{2}\right)+G\left(y, s_{1}\right)\right\| \leq\|x-y\|\left(h\left(s_{2}\right)-h\left(s_{1}\right)\right)
$$

para $x, y \in P C_{1}$ e $t_{0} \leq s_{1}<s_{2} \leq t_{0}+\sigma$. Logo, as desigualdades (3.18) e (3.19) mostram que a função $G$ dada por (3.17) pertence à classe $\mathscr{F}(\Omega, h)$, onde $\Omega=P C_{1} \times\left[t_{0}, t_{0}+\sigma\right]$.

Suponhamos que as funções $f$ e $I_{k}$ da equação (3.1) satisfaçam as condições $(A),(B),\left(A^{\prime}\right)$ e $\left(B^{\prime}\right)$ da seção anterior e que as superfícies $\tau_{k}$ satisfaçam as condições $(C 1)$ até $(C 5)$. Consideraremos, então, a EDO generalizada

$$
\frac{d x}{d \tau}=D G(x, t),
$$

onde a função $G$ é dada por (3.17). Vamos estudar as relações entre a EDFR com impulsos em tempo variável (3.1) e a EDO generalizada (3.20).

Na sequência, adaptaremos para EDFRs com impulsos em tempo variável, resultados extraídos de [8] para EDFRs com impulsos em tempo pré-fixado.

Lema 3.4. Seja x uma solução de (3.20) no intervalo $\left[t_{0}, t_{0}+\sigma\right]$, com $G$ dada por (3.17) e com condição inicial $x\left(t_{0}\right) \in P C_{1}$ dada por $x\left(t_{0}\right)(\vartheta)=\phi(\vartheta)$ para $\vartheta \in\left[t_{0}-r, t_{0}\right]$ e $x\left(t_{0}\right)(\vartheta)=x\left(t_{0}\right)\left(t_{0}\right)$ para $\vartheta \in$ $\left[t_{0}, t_{0}+\sigma\right]$. Então se $v \in\left[t_{0}, t_{0}+\sigma\right]$, teremos

$$
x(v)(\vartheta)=x(v)(v), \quad \vartheta \geq v, \vartheta \in\left[t_{0}, t_{0}+\sigma\right]
$$

$e$

$$
x(v)(\vartheta)=x(\vartheta)(\vartheta), \quad v \geq \vartheta, \vartheta \in\left[t_{0}, t_{0}+\sigma\right]
$$


Demonstração. Suponhamos, inicialmente, que $\vartheta \geq v$. Como $x$ é solução de (3.20), temos

$$
x(v)(v)=x\left(t_{0}\right)(v)+\int_{t_{0}}^{v} D G(x(\tau), t)(v)
$$

e analogamente,

$$
x(v)(\vartheta)=x\left(t_{0}\right)(\vartheta)+\int_{t_{0}}^{v} D G(x(\tau), t)(\vartheta) .
$$

Mas, $x\left(t_{0}\right)(\vartheta)=x\left(t_{0}\right)(v)$, pelas propriedades da condição inicial, logo

$$
x(v)(\vartheta)-x(v)(v)=\int_{t_{0}}^{v} D G(x(\tau), t)(\vartheta)-\int_{t_{0}}^{v} D G(x(\tau), t)(v) .
$$

Como a integral $\int_{t_{0}}^{v} D G(x(\tau), t)$ existe, para todo $\varepsilon>0$ existe um calibre $\delta$ em $\left[t_{0}, t_{0}+\sigma\right]$ tal que se $\left(\xi_{n},\left[s_{n-1}, s_{n}\right]\right)$ for uma divisão $\delta$-fina de $\left[t_{0}, v\right]$, então

$$
\left\|\sum_{n}\left[G\left(x\left(\xi_{n}\right), s_{n}\right)-G\left(x\left(\xi_{n}\right), s_{n-1}\right)\right]-\int_{t_{0}}^{v} D G(x(\xi), t)\right\|<\varepsilon .
$$

Portanto, temos

$$
\begin{gathered}
|x(v)(\vartheta)-x(v)(v)|<2 \varepsilon+ \\
+\left|\sum_{n}\left[G\left(x\left(\xi_{n}\right), s_{n}\right)-G\left(x\left(\xi_{n}\right), s_{n-1}\right)\right](\vartheta)-\sum_{n}\left[G\left(x\left(\xi_{n}\right), s_{n}\right)-G\left(x\left(\xi_{n}\right), s_{n-1}\right)\right](v)\right| .
\end{gathered}
$$

Pela definição da função $G$ dada em (3.17), pela forma de $F$ dada em (3.3) e de $J$ dada em (3.10), podemos constatar que, para todo $n$,

$$
G\left(x\left(\xi_{n}\right), s_{n}\right)(\vartheta)-G\left(x\left(\xi_{n}\right), s_{n-1}\right)(\vartheta)=G\left(x\left(\xi_{n}\right), s_{n}\right)(v)-G\left(x\left(\xi_{n}\right), s_{n-1}\right)(v) .
$$

De fato, como $t_{0} \leq s_{n-1} \leq s_{n} \leq v \leq \vartheta$ para todo $n$, temos

$$
F\left(x\left(\xi_{n}\right), s_{n}\right)(\vartheta)-F\left(x\left(\xi_{n}\right), s_{n-1}\right)(\vartheta)=\int_{s_{n-1}}^{s_{n}} f\left(x\left(\xi_{n}\right)_{s}, s\right) d s
$$

$\mathrm{e}$

$$
F\left(x\left(\xi_{n}\right), s_{n}\right)(v)-F\left(x\left(\xi_{n}\right), s_{n-1}\right)(v)=\int_{s_{n-1}}^{s_{n}} f\left(x\left(\xi_{n}\right)_{s}, s\right) d s
$$

Logo,

$$
F\left(x\left(\xi_{n}\right), s_{n}\right)(\vartheta)-F\left(x\left(\xi_{n}\right), s_{n-1}\right)(\vartheta)=F\left(x\left(\xi_{n}\right), s_{n}\right)(v)-F\left(x\left(\xi_{n}\right), s_{n-1}\right)(v) .
$$

Agora, fixe $i \in\left\{1, \ldots, m\left(\tau_{k}\right)\right\}$ e $k \in\{1,2, \ldots\}$. Note que se $t_{0} \leq \vartheta \leq t_{k}^{i}$, então $t_{0} \leq v \leq t_{k}^{i}$, o que implica 
$H_{k}^{i}(\vartheta)=H_{k}^{i}(v)=0 \mathrm{e}$, portanto

$$
\left[H_{k}^{i}\left(s_{n}\right)-H_{k}^{i}\left(s_{n-1}\right)\right] H_{k}^{i}(\vartheta) I_{k}\left(y\left(t_{k}^{i}\right)\right)=0=\left[H_{k}^{i}\left(s_{n}\right)-H_{k}^{i}\left(s_{n-1}\right)\right] H_{k}^{i}(v) I_{k}\left(y\left(t_{k}^{i}\right)\right)
$$

Por outro lado, se $\vartheta>t_{k}^{i}$ e $t_{0} \leq v \leq t_{k}^{i}$, temos $H_{k}^{i}(\vartheta)=1$ e $H_{k}^{i}(v)=H_{k}^{i}\left(s_{n}\right)=H_{k}^{i}\left(s_{n-1}\right)=0$ e, consequentemente,

$$
\left[H_{k}^{i}\left(s_{n}\right)-H_{k}^{i}\left(s_{n-1}\right)\right] H_{k}^{i}(\vartheta) I_{k}\left(y\left(t_{k}^{i}\right)\right)=0=\left[H_{k}^{i}\left(s_{n}\right)-H_{k}^{i}\left(s_{n-1}\right)\right] H_{k}^{i}(v) I_{k}\left(y\left(t_{k}^{i}\right)\right),
$$

enquanto que, se $\vartheta>t_{k}^{i}$ e $v>t_{k}^{i}$ então $H_{k}^{i}(\vartheta)=H_{k}^{i}(v)=1$, daí

$$
\left[H_{k}^{i}\left(s_{n}\right)-H_{k}^{i}\left(s_{n-1}\right)\right] H_{k}^{i}(\vartheta) I_{k}\left(y\left(t_{k}^{i}\right)\right)=\left[H_{k}^{i}\left(s_{n}\right)-H_{k}^{i}\left(s_{n-1}\right)\right] H_{k}^{i}(v) I_{k}\left(y\left(t_{k}^{i}\right)\right)
$$

donde concluímos que

$$
J\left(x\left(\xi_{n}\right), s_{n}\right)(\vartheta)-J\left(x\left(\xi_{n}\right), s_{n-1}\right)(\vartheta)=J\left(x\left(\xi_{n}\right), s_{n}\right)(v)-J\left(x\left(\xi_{n}\right), s_{n-1}\right)(v) .
$$

Portanto, por (3.25) e (3.26), a igualdade (3.24) se verifica.

Logo, pela desigualdade (3.23), temos

$$
|x(v)(\vartheta)-x(v)(v)|<2 \varepsilon .
$$

Como $\varepsilon>0$ pode ser tomado arbitrariamente pequeno, obtemos o resultado desejado.

Agora, suponhamos que $\vartheta \leq v$. Pela definição de uma solução de (3.20), como na primeira parte da demonstração, temos

$$
x(v)(\vartheta)=x\left(t_{0}\right)(\vartheta)+\int_{t_{0}}^{v} D G(x(\tau), t)(\vartheta)
$$

$\mathrm{e}$

$$
x(\vartheta)(\vartheta)=x\left(t_{0}\right)(\vartheta)+\int_{t_{0}}^{\vartheta} D G(x(\tau), t)(\vartheta)
$$

Portanto,

$$
x(v)(\vartheta)-x(\vartheta)(\vartheta)=\int_{\vartheta}^{v} D G(x(\tau), t)(\vartheta)
$$

Agora, se $\left(\xi_{n},\left[s_{n-1}, s_{n}\right]\right)$ for uma divisão marcada de $[\vartheta, v]$, novamente, podemos verificar por (3.3) 
e (3.10) que, para todo $n$,

$$
G\left(x\left(\xi_{n}\right), s_{n}\right)(\vartheta)-G\left(x\left(\xi_{n}\right), s_{n-1}\right)(\vartheta)=0 .
$$

Logo $\int_{\vartheta}^{v} D G(x(\tau), t)(\vartheta)=0$ e, portanto, $x(v)(\vartheta)=x(\vartheta)(\vartheta)$, donde segue o resultado.

Teorema 3.5. Considere o sistema (3.1)-(3.2), onde para todo $y \in P C_{1}$, a função $f: G^{-}\left([-r, 0], \mathbb{R}^{n}\right) \times$ $\left[t_{0}, t_{0}+\sigma\right] \rightarrow \mathbb{R}^{n}$ é tal que $t \mapsto f\left(y_{t}, t\right)$ é Lebesgue integrável em $\left[t_{0}, t_{0}+\sigma\right]$ e as condições $(C 1)$ até $(C 5)$ e (A), (B), ( $\left.A^{\prime}\right),\left(B^{\prime}\right)$ estão satisfeitas. Seja y uma solução do problema (3.1)-(3.2) no intervalo $\left[t_{0}-r, t_{0}+\sigma\right]$. Dado $t \in\left[t_{0}, t_{0}+\sigma\right]$, seja

$$
x(t)(\vartheta)=\left\{\begin{array}{l}
y(\vartheta), \vartheta \in\left[t_{0}-r, t\right] \\
y(t), \vartheta \in\left[t, t_{0}+\sigma\right]
\end{array}\right.
$$

Então $x(t) \in G^{-}\left(\left[t_{0}-r, t_{0}+\sigma\right], \mathbb{R}^{n}\right)$ e x será uma solução de (3.20) em $\left[t_{0}, t_{0}+\sigma\right]$ com condição inicial

$$
x\left(t_{0}\right)(\vartheta)=\left\{\begin{array}{l}
\phi(\vartheta), \vartheta \in\left[t_{0}-r, t_{0}\right] \\
y(t), \vartheta \in\left[t_{0}, t_{0}+\sigma\right]
\end{array}\right.
$$

Demonstração. Vamos mostrar que, para todo $v \in\left[t_{0}, t_{0}+\sigma\right]$, a integral $\int_{t_{0}}^{v} D G(x(\tau), t)$ existe e vale

$$
x(v)-x\left(t_{0}\right)=\int_{t_{0}}^{v} D G(x(\tau), t)
$$

Seja $\varepsilon>0$. Como y é uma solução de (3.1), a relação

$$
y(t)=\phi(0)+\int_{t_{0}}^{t} f\left(y_{s}, s\right) d s+\sum_{\substack{t_{0} \leq t_{k}^{i}<t, i=1, \ldots, m\left(\tau_{k}\right)}} I_{k}\left(y\left(t_{k}^{i}\right)\right), \quad t \in\left[t_{0}, t_{0}+\sigma\right], k=0, \ldots, j
$$

onde $j$ é tal que $t_{0} \equiv \tau_{0}(x)<\tau_{1}(x)<\tau_{2}(x)<\ldots<\tau_{j}(x) \leq t_{0}+\sigma$, referente à forma integral correspondente está satisfeita e a função $y:\left[t_{0}, t_{0}+\sigma\right] \rightarrow \mathbb{R}^{n}$ pode ser escrita como a soma de uma função absolutamente contínua e uma função escada finita contínua à esquerda.

Desta forma, para todo $\tau \in\left[t_{0}, t_{0}+\sigma\right]$, existe um $\delta(\tau)>0$ tal que

$$
|y(\rho)-y(\tau)|<\varepsilon \text { para todo } \rho \in[\tau-\delta(\tau), \tau]
$$

e

$$
|y(\rho)-y(\tau+)|<\varepsilon \text { para todo } \rho \in(\tau, \tau+\delta(\tau)] .
$$


Portanto, temos naturalmente um calibre $\delta$ de $\left[t_{0}, t_{0}+\sigma\right]$. Mas, sem perda de generalidade, podemos considerar o calibre $\delta$ de modo que, se $\tau \in\left[t_{0}, t_{0}+\sigma\right]$, então

$$
\left|\int_{u}^{v} L(s) d s\right|<\frac{\varepsilon}{\left(\left(1+m\left(\tau_{1}\right)+\ldots+m\left(\tau_{j}\right)\right)+1\right)\left(K_{1}+1\right)} \text {, para todo }[u, v] \subset(\tau-\delta(\tau), \tau+\delta(\tau)),
$$

onde $1+m\left(\tau_{1}\right)+\ldots+m\left(\tau_{j}\right)$ é o número de impulsos no intervalo $\left[t_{0}, t_{0}+\sigma\right]$, se $t_{0}$ for um momento de impulso (se $t_{0}$ não for um momento de impulso do sistema (3.1)-(3.2), procedemos de forma análoga para provar o presente resultado, considerando $m\left(\tau_{1}\right)+\ldots+m\left(\tau_{j}\right)$ o número de impulsos no intervalo $\left.\left[t_{0}, t_{0}+\sigma\right]\right)$ e $K_{1}$ é a constante da condição $\left(A^{\prime}\right)$. Esta escolha é possível porque a função $L:\left[t_{0}, t_{0}+\sigma\right] \rightarrow$ $\mathbb{R}$ da condição $(B)$ é Lebesgue integrável. Suponhamos, ainda, que o calibre $\delta$ satisfaça as seguintes condições

$$
0<\delta(\tau)<\min \left\{\frac{t_{k}^{i+1}-t_{k}^{i}}{2}, \frac{t_{k}^{1}-t_{k-1}^{m\left(\tau_{k-1}\right)}}{2} ; k=1, \ldots, j, i=1, \ldots, m\left(\tau_{k}\right)-1\right\}
$$

$\mathrm{e}$

$0<\delta(\tau)<\min \left\{\left|\tau-t_{k}^{i+1}\right|,\left|\tau-t_{k}^{i}\right| ; \tau \in\left(t_{k}^{i}, t_{k}^{i+1}\right)\right.$ ou $\left.\tau \in\left(t_{k-1}^{m\left(\tau_{k-1}\right)}, t_{k}^{1}\right), k=1, \ldots, j, i=1, \ldots, m\left(\tau_{k}\right)-1\right\}$

A condição (3.32) assegura que se um intervalo marcado $\left(T,\left[s_{1}, s_{2}\right]\right)$ for $\delta$-fino, então o intervalo $\left[s_{1}, s_{2}\right]$ conterá no máximo um ponto $t_{k}^{i}$ para $k=0, \ldots, j$ e $i=1, \ldots, m\left(\tau_{k}\right)$, enquanto (3.33) implica que $T=t_{k}^{i}$ sempre que $t_{k}^{i} \in\left[s_{1}, s_{2}\right]$.

Suponhamos que $\left(\xi_{n},\left[s_{n-1}, s_{n}\right]\right)$ seja uma partição $\delta$-fina do intervalo $\left[t_{0}, v\right]$. Usando a definição (3.27) de $x$ e (3.28), podemos mostrar facilmente que

$$
\begin{aligned}
& {\left[x\left(s_{n}\right)-x\left(s_{n-1}\right)\right](\vartheta)=} \\
& =\left\{\begin{array}{l}
0, \vartheta \in\left[t_{0}-r, s_{n-1}\right] \\
\int_{s_{n-1}}^{\vartheta} f\left(y_{s}, s\right) d s+\sum_{k=0}^{j} \sum_{i=1}^{m\left(\tau_{k}\right)} I_{k}\left(y\left(t_{k}^{i}\right)\right)\left[H_{k}^{i}(\vartheta)-H_{k}^{i}\left(s_{n-1}\right)\right], \vartheta \in\left[s_{n-1}, s_{n}\right], \\
\int_{s_{n-1}}^{s_{n}} f\left(y_{s}, s\right) d s+\sum_{k=0}^{j} \sum_{i=1}^{m\left(\tau_{k}\right)} I_{k}\left(y\left(t_{k}^{i}\right)\right)\left[H_{k}^{i}\left(s_{n}\right)-H_{k}^{i}\left(s_{n-1}\right)\right], \vartheta \in\left[s_{n}, t_{0}+\sigma\right] .
\end{array}\right.
\end{aligned}
$$

Usando a definição de $G$ dada em (3.17) e usando (3.3) e (3.10), obtemos

$$
\left[G\left(x\left(\xi_{n}\right), s_{n}\right)-G\left(x\left(\xi_{n}\right), s_{n-1}\right)\right](\vartheta)=
$$




$$
\begin{aligned}
=\left[F\left(x\left(\xi_{n}\right), s_{n}\right)-F\right. & \left.\left.x\left(\xi_{n}\right), s_{n-1}\right)\right](\vartheta)+\sum_{k=0}^{j} \sum_{i=1}^{m\left(\tau_{k}\right)}\left[H_{k}^{i}\left(s_{n}\right)-H_{k}^{i}\left(s_{n-1}\right)\right] H_{k}^{i}(\vartheta) I_{k}\left(x\left(\tau_{n}\right)\left(t_{k}^{i}\right)\right)= \\
= & \left\{\begin{array}{l}
0, \vartheta \in\left[t_{0}-r, s_{n-1}\right] \\
\int_{s_{n-1}}^{\vartheta} f\left(x\left(\xi_{n}\right)_{s}, s\right) d s, \vartheta \in\left[s_{n-1}, s_{n}\right] \\
\int_{s_{n-1}}^{s_{n}} f\left(x\left(\xi_{n}\right)_{s}, s\right) d s, \vartheta \in\left[s_{n}, t_{0}+\sigma\right]
\end{array}\right\}+ \\
+ & \sum_{k=0}^{j} \sum_{i=1}^{m\left(\tau_{k}\right)}\left[H_{k}^{i}\left(s_{n}\right)-H_{k}^{i}\left(s_{n-1}\right)\right] H_{k}^{i}(\vartheta) I_{k}\left(x\left(\xi_{n}\right)\left(t_{k}^{i}\right)\right) .
\end{aligned}
$$

Usando as propriedades (3.32) e (3.33) do calibre $\delta$ e as propriedades correspondentes da partição $\left(\xi_{n},\left[s_{n-1}, s_{n}\right]\right)$, existem duas possibilidades para um intervalo marcado $\left(\xi_{n},\left[s_{n-1}, s_{n}\right]\right)$ :

(i) existe exatamente um $t_{l}^{i} \in\left[s_{n-1}, s_{n}\right)$,

(ii) $\left[s_{n-1}, s_{n}\right)$ não contém ponto de impulso, isto é, $\left[s_{n-1}, s_{n}\right) \cap\left\{t_{0}, t_{1}^{1}, \ldots, t_{1}^{m\left(\tau_{1}\right)}, \ldots, t_{j}^{1}, \ldots, t_{j}^{m\left(\tau_{j}\right)}\right\}=\emptyset$.

No caso $(i)$, temos

$$
\sum_{k=0}^{j} \sum_{i=1}^{m\left(\tau_{k}\right)} I_{k}\left(y\left(t_{k}^{i}\right)\right)\left[H_{k}^{i}(\vartheta)-H_{k}^{i}\left(s_{n-1}\right)\right]=I_{l}\left(y\left(t_{l}^{i}\right)\right) H_{l}^{i}(\vartheta)
$$

e, como $\xi_{n}=t_{l}^{i}$, obtemos, pela definição de $x$,

$$
\sum_{k=0}^{j} \sum_{i=1}^{m\left(\tau_{k}\right)}\left[H_{k}^{i}\left(s_{n}\right)-H_{k}^{i}\left(s_{n-1}\right)\right] H_{k}^{i}(\vartheta) I_{k}\left(x\left(\xi_{n}\right)\left(t_{k}^{i}\right)\right)=I_{l}\left(x\left(\xi_{n}\right)\left(t_{l}^{i}\right)\right) H_{l}^{i}(\vartheta)=I_{l}\left(y\left(t_{l}^{i}\right)\right) H_{l}^{i}(\vartheta)
$$

Daí, por (3.34) e (3.35), temos

$$
\begin{aligned}
& {\left[x\left(s_{n}\right)-x\left(s_{n-1}\right)\right](\vartheta)-\left[G\left(x\left(\xi_{n}\right), s_{n}\right)-G\left(x\left(\xi_{n}\right), s_{n-1}\right)\right](\vartheta)=} \\
& =\left\{\begin{array}{l}
0, \vartheta \in\left[t_{0}-r, s_{n-1}\right] \\
\int_{s_{n-1}}^{\vartheta} f\left(y_{s}, s\right) d s-\int_{s_{n-1}}^{\vartheta} f\left(x\left(\xi_{n}\right)_{s}, s\right) d s, \vartheta \in\left[s_{n-1}, s_{n}\right],= \\
\int_{s_{n-1}}^{s_{n}} f\left(y_{s}, s\right) d s-\int_{s_{n-1}}^{s_{n}} f\left(x\left(\xi_{n}\right)_{s}, s\right) d s, \vartheta \in\left[s_{n}, t_{0}+\sigma\right]
\end{array}\right.
\end{aligned}
$$




$$
=\left\{\begin{array}{l}
0, \vartheta \in\left[t_{0}-r, s_{n-1}\right] \\
\int_{s_{n-1}}^{\vartheta}\left[f\left(y_{s}, s\right)-f\left(x\left(\xi_{n}\right)_{s}, s\right)\right] d s, \vartheta \in\left[s_{n-1}, s_{n}\right], \\
\int_{s_{n-1}}^{s_{n}}\left[f\left(y_{s}, s\right)-f\left(x\left(\xi_{n}\right)_{s}, s\right)\right] d s, \vartheta \in\left[s_{n}, t_{0}+\sigma\right] .
\end{array}\right.
$$

No caso (ii), temos

$$
\sum_{k=0}^{j} \sum_{i=1}^{m\left(\tau_{k}\right)} I_{k}\left(y\left(t_{k}^{i}\right)\right)\left[H_{k}^{i}(\vartheta)-H_{k}^{i}\left(s_{n-1}\right)\right]=0
$$

e também

$$
\sum_{k=0}^{j} \sum_{i=1}^{m\left(\tau_{k}\right)}\left[H_{k}^{i}\left(s_{n}\right)-H_{k}^{i}\left(s_{n-1}\right)\right] H_{k}^{i}(\vartheta) I_{k}\left(x\left(\xi_{n}\right)\left(t_{k}^{i}\right)\right)=0 .
$$

Então, por (3.34) e (3.35), obtemos a relação (3.36).

Usando (3.36), também temos

$$
\begin{gathered}
\left\|x\left(s_{n}\right)-x\left(s_{n-1}\right)-\left[G\left(x\left(\xi_{n}\right), s_{n}\right)-G\left(x\left(\xi_{n}\right), s_{n-1}\right)\right]\right\|= \\
=\sup _{\vartheta \in\left[t_{0}-r, t_{0}+\sigma\right]}\left|\left[x\left(s_{n}\right)-x\left(s_{n-1}\right)\right](\vartheta)-\left[G\left(x\left(\xi_{n}\right), s_{n}\right)-G\left(x\left(\xi_{n}\right), s_{n-1}\right)\right](\vartheta)\right|= \\
=\sup _{\vartheta \in\left[t_{0}-r, t_{0}+\sigma\right]}\left\{\begin{array}{l}
\left|\int_{s_{n-1}}^{\vartheta}\left[f\left(y_{s}, s\right)-f\left(x\left(\xi_{n}\right)_{s}, s\right)\right] d s\right|, \vartheta \in\left[s_{n-1}, s_{n}\right] \\
\left|\int_{s_{n-1}}^{s_{n}}\left[f\left(y_{s}, s\right)-f\left(x\left(\xi_{n}\right)_{s}, s\right)\right] d s\right|, \vartheta \in\left[s_{n}, t_{0}+\sigma\right]
\end{array}\right. \\
=\sup _{\vartheta \in\left[s_{n-1}, s_{n}\right]}\left|\int_{s_{n-1}}^{\vartheta}\left[f\left(y_{s}, s\right)-f\left(x\left(\xi_{n}\right)_{s}, s\right)\right] d s\right| .
\end{gathered}
$$

Pela definição de $x$ dada por (3.27), temos para o caso $(i)$,

$$
\int_{s_{n-1}}^{\vartheta}\left[f\left(y_{s}, s\right)-f\left(x\left(\xi_{n}\right)_{s}, s\right)\right] d s=\int_{s_{n-1}}^{\vartheta}\left[f\left(y_{s}, s\right)-f\left(x\left(t_{l}^{i}\right)_{s}, s\right)\right] d s=\int_{t_{l}^{i}}^{\vartheta}\left[f\left(y_{s}, s\right)-f\left(x\left(t_{l}^{i}\right)_{s}, s\right)\right] d s,
$$

para $\vartheta \in\left[t_{l}^{i}, s_{n}\right]$, uma vez que $\int_{s_{n-1}}^{t_{l}^{i}}\left[f\left(y_{s}, s\right)-f\left(x\left(t_{l}^{i}\right)_{s}, s\right)\right] d s=0$. E também vale

$$
\int_{s_{n-1}}^{\vartheta}\left[f\left(y_{s}, s\right)-f\left(x\left(\xi_{n}\right)_{s}, s\right)\right] d s=0
$$

para $\vartheta \in\left[s_{n-1}, t_{l}^{i}\right]$. Pela condição $(B)$, temos

$$
\left|\int_{t_{l}^{i}}^{\vartheta}\left[f\left(y_{s}, s\right)-f\left(x\left(t_{l}^{i}\right)_{s}, s\right)\right] d s\right| \leq \int_{t_{l}^{i}}^{\vartheta} L(s)\left\|y_{s}-x\left(t_{l}^{i}\right)_{s}\right\| d s, \vartheta \in\left[t_{l}^{i}, s_{n}\right] .
$$


Usando (3.30) e $\left(B^{\prime}\right)$, então para $s \in\left[t_{l}^{i}, s_{n}\right]$, temos

$$
\begin{gathered}
\left\|y_{s}-x\left(t_{l}^{i}\right)_{s}\right\|=\sup _{\rho \in[-r, 0]}\left|y(s+\rho)-x\left(t_{l}^{i}\right)(s+\rho)\right|= \\
=\sup _{\rho \in\left[t_{l}^{i}, s\right]}\left|y(\rho)-y\left(t_{l}^{i}\right)\right|=\sup _{\rho \in\left[t_{l}^{i}, s\right]}\left|y(\rho)-y\left(t_{l}^{i}+\right)+y\left(t_{l}^{i}+\right)-y\left(t_{l}^{i}\right)\right|= \\
=\sup _{\rho \in\left[t_{l}^{i}, s\right]}\left|y(\rho)-y\left(t_{l}^{i}+\right)+I_{l} y\left(t_{l}^{i}\right)\right| \leq \varepsilon+K_{1} .
\end{gathered}
$$

Portanto, pela propriedade (3.31) do calibre $\delta$, temos

$$
\begin{gathered}
\left\|x\left(s_{n}\right)-x\left(s_{n-1}\right)-\left[G\left(x\left(\xi_{n}\right), s_{n}\right)-G\left(x\left(\xi_{n}\right), s_{n-1}\right)\right]\right\| \leq\left(\varepsilon+K_{1}\right) \int_{t_{l}^{i}}^{s_{n}} L(s) d s \leq \\
\leq \varepsilon \int_{t_{l}^{i}}^{s_{n}} L(s) d s+K_{1} \frac{\varepsilon}{\left(\left(1+m\left(\tau_{1}\right)+\ldots+m\left(\tau_{j}\right)\right)+1\right)\left(K_{1}+1\right)} \\
<\varepsilon \int_{t_{l}^{i}}^{s_{n}} L(s) d s+\frac{\varepsilon}{\left(\left(1+m\left(\tau_{1}\right)+\ldots+m\left(\tau_{j}\right)\right)+1\right)} .
\end{gathered}
$$

Analogamente, para o caso (ii), temos

$$
\int_{s_{n-1}}^{\vartheta}\left[f\left(y_{s}, s\right)-f\left(x\left(\xi_{n}\right)_{s}, s\right)\right] d s=\int_{\xi_{n}}^{\vartheta}\left[f\left(y_{s}, s\right)-f\left(x\left(\xi_{n}\right)_{s}, s\right)\right] d s
$$

para $\vartheta \in\left[\xi_{n}, s_{n}\right] \mathrm{e}$

$$
\int_{s_{n-1}}^{\vartheta}\left[f\left(y_{s}, s\right)-f\left(x\left(\xi_{n}\right)_{s}, s\right)\right] d s=0
$$

para $\vartheta \in\left[s_{n-1}, \xi_{n}\right]$.

Pela condição $(B)$, temos

$$
\left|\int_{\xi_{n}}^{\vartheta}\left[f\left(y_{s}, s\right)-f\left(x\left(\xi_{n}\right)_{s}, s\right)\right] d s\right| \leq \int_{\xi_{n}}^{\vartheta} L(s)\left\|y_{s}-x\left(\xi_{n}\right)_{s}\right\| d s, \vartheta \in\left[\xi_{n}, s_{n}\right] .
$$

onde

$$
\left\|y_{s}-x\left(\xi_{n}\right)_{s}\right\|=\sup _{\rho \in\left[\xi_{n}, s\right]}\left|y(\rho)-y\left(\xi_{n}\right)\right| \leq \varepsilon, s \in\left[\xi_{n}, s_{n}\right],
$$

pela propriedade do calibre $\delta$ dada em (3.29) .

Logo,

$$
\left\|x\left(s_{n}\right)-x\left(s_{n-1}\right)-\left[G\left(x\left(\xi_{n}\right), s_{n}\right)-G\left(x\left(\xi_{n}\right), s_{n-1}\right)\right]\right\| \leq \varepsilon \int_{\xi_{n}}^{s_{n}} L(s) d s .
$$


Usando os resultados obtidos acima e o fato de que o caso $(i)$ ocorre em no máximo $j+1$ intervalos, temos

$$
\begin{gathered}
\left\|x(v)-x\left(t_{0}\right)-\sum_{n}\left[G\left(x\left(\xi_{n}\right), s_{n}\right)-G\left(x\left(\xi_{n}\right), s_{n-1}\right)\right]\right\|= \\
=\left\|\sum_{n}\left\{x\left(s_{n}\right)-x\left(s_{n-1}\right)-\left[G\left(x\left(\xi_{n}\right), s_{n}\right)-G\left(x\left(\xi_{n}\right), s_{n-1}\right)\right]\right\}\right\| \leq \\
\leq \sum_{n}\left\|x\left(s_{n}\right)-x\left(s_{n-1}\right)-\left[G\left(x\left(\xi_{n}\right), s_{n}\right)-G\left(x\left(\xi_{n}\right), s_{n-1}\right)\right]\right\| \leq \\
\leq \sum_{n ; t_{l}^{i} \in\left[s_{n-1}, s_{n}\right)}\left[\varepsilon \int_{t_{l}^{i}}^{s_{n}} L(s) d s+\frac{\varepsilon}{\left(\left(1+m\left(\tau_{1}\right)+\ldots+m\left(\tau_{j}\right)\right)+1\right)}\right]+\sum_{j} \varepsilon \int_{\xi_{n}}^{s_{n}} L(s) d s< \\
<2 \varepsilon \int_{t_{0}}^{t_{0}+\sigma} L(s) d s+(j+1) \frac{\varepsilon}{\left(\left(1+m\left(\tau_{1}\right)+\ldots+m\left(\tau_{j}\right)\right)+1\right)}<2 \varepsilon \int_{t_{0}}^{t_{0}+\sigma} L(s) d s+\varepsilon
\end{gathered}
$$

Portanto, para todo $v \in\left[t_{0}, t_{0}+\sigma\right]$ a integral $\int_{t_{0}}^{v} D G(x(\tau), t)$ existe $\mathrm{e}$

$$
x(v)-x\left(t_{0}\right)=\int_{t_{0}}^{v} D G(x(\tau), t)
$$

donde segue o resultado.

Teorema 3.6. Seja G dada por (3.17) e seja x uma solução de (3.20) no intervalo $\left[t_{0}, t_{0}+\sigma\right]$ satisfazendo a condição inicial

$$
x\left(t_{0}\right)(\vartheta)=\left\{\begin{array}{l}
\phi\left(\vartheta-t_{0}\right), t_{0}-r \leq \vartheta \leq t_{0} \\
x\left(t_{0}\right)\left(t_{0}\right), t_{0} \leq \vartheta \leq t_{0}+\sigma
\end{array}\right.
$$

onde $\phi \in G^{-}\left([-r, 0], \mathbb{R}^{n}\right)$. Para todo $\vartheta \in\left[t_{0}-r, t_{0}+\sigma\right]$, consideremos

$$
y(\vartheta)=\left\{\begin{array}{l}
x\left(t_{0}\right)(\vartheta), t_{0}-r \leq \vartheta \leq t_{0} \\
x(\vartheta)(\vartheta), t_{0} \leq \vartheta \leq t_{0}+\sigma .
\end{array}\right.
$$

Então y é uma solução do sistema (3.1)-(3.2) em $\left[t_{0}-r, t_{0}+\sigma\right]$.

Demonstração. De acordo com (3.28), precisamos provar que, para todo $\eta>0$ e para todo $v \in\left[t_{0}, t_{0}+\sigma\right]$, temos

$$
\left|y(v)-y\left(t_{0}\right)-\int_{t_{0}}^{v} f\left(y_{s}, s\right) d s-\sum_{k=0}^{j} \sum_{i=1}^{m\left(\tau_{k}\right)} I_{k}\left(y\left(t_{k}^{i}\right)\right) H_{k}^{i}(v)\right|<\eta
$$

$\mathrm{e}$

$$
y_{t_{0}}=\phi
$$


Lembramos ao leitor que estamos considerando que $t_{0}$ pode ser um momento de impulso do sistema (3.1)-(3.2). Caso $t_{0}$ não seja um momento de impulso, consideramos $I_{0} \equiv 0$, como foi mencionado na seção anterior.

Suponhamos que um calibre $\delta:\left[t_{0}, t_{0}+\sigma\right] \rightarrow(0,+\infty)$ satisfaça, para $\tau \in\left[t_{0}, t_{0}+\sigma\right]$, as seguintes desigualdades:

$$
0<\delta(\tau)<\min \left\{\frac{t_{k}^{i+1}-t_{k}^{i}}{2}, \frac{t_{k}^{1}-t_{k-1}^{m\left(\tau_{k-1}\right)}}{2} ; k=1, \ldots, j, i=1, \ldots, m\left(\tau_{k}\right)-1\right\}
$$

$\mathrm{e}$

$0<\delta(\tau)<\min \left\{\left|\tau-t_{k}^{i+1}\right|,\left|\tau-t_{k}^{i}\right| ; \tau \in\left(t_{k}^{i}, t_{k}^{i+1}\right)\right.$ ou $\left.\tau \in\left(t_{k-1}^{m\left(\tau_{k-1}\right)}, t_{k}^{1}\right), k=1, \ldots, j, i=1, \ldots, m\left(\tau_{k}\right)-1\right\}$

Como na prova do Teorema 3.5, a condição (3.39) assegura que se um intervalo marcado $\left(T,\left[s_{1}, s_{2}\right]\right)$ for $\delta$-fino, então o intervalo $\left[s_{1}, s_{2}\right]$ conterá no máximo um dos pontos $t_{k}^{i}, k=0, \ldots, j, i=1, \ldots, m\left(\tau_{k}\right)$, enquanto (3.40) implica que $T=t_{k}^{i}$ para $t_{k}^{i} \in\left[s_{1}, s_{2}\right]$.

$\operatorname{Se}\left(\xi_{n},\left[s_{n-1}, s_{n}\right]\right)$ for uma partição $\delta$-fina de $\left[t_{0}, v\right]$, então por (3.11), quando $t_{l}^{i} \in\left[s_{n-1}, s_{n}\right)$, teremos

$$
\begin{gathered}
J\left(x\left(\xi_{n}\right), s_{n}\right)(\vartheta)-J\left(x\left(\xi_{n}\right), s_{n-1}\right)(\vartheta)=\sum_{k=0}^{j} \sum_{i=1}^{m\left(\tau_{k}\right)}\left[H_{k}^{i}\left(s_{n}\right)-H_{k}^{i}\left(s_{n-1}\right)\right] H_{k}^{i}(\vartheta) I_{k}\left(x\left(\xi_{n}\right)\left(t_{k}^{i}\right)\right)= \\
=H_{l}^{i}(\vartheta) I_{k}\left(x\left(t_{l}^{i}\right)\left(t_{l}^{i}\right)\right)=H_{l}^{i}(\vartheta) I_{k}\left(y\left(t_{l}^{i}\right)\right)
\end{gathered}
$$

para $\vartheta \in\left[t_{0}-r, t_{0}+\sigma\right]$, e se $\left[s_{n-1}, s_{n}\right)$ não contiver algum dos pontos $t_{0}, t_{1}^{1}, \ldots, t_{1}^{m\left(\tau_{1}\right)}, \ldots, t_{j}^{1}, \ldots, t_{j}^{m\left(\tau_{j}\right)}$, então

$$
J\left(x\left(\xi_{n}\right), s_{n}\right)(\vartheta)-J\left(x\left(\xi_{n}\right), s_{n-1}\right)(\vartheta)=\sum_{k=0}^{j} \sum_{i=1}^{m\left(\tau_{k}\right)}\left[H_{k}^{i}\left(s_{n}\right)-H_{k}^{i}\left(s_{n-1}\right)\right] H_{k}^{i}(\vartheta) I_{k}\left(x\left(\xi_{n}\right)\left(t_{k}^{i}\right)\right)=0 .
$$

Isto implica que a integral $\int_{t_{0}}^{v} D J(x(\tau), t)$ existe $\mathrm{e}$

$$
\left[\int_{t_{0}}^{v} D J(x(\tau), t)\right](v)=\sum_{k=0}^{j} \sum_{i=1}^{m\left(\tau_{k}\right)} H_{k}^{i}(v) I_{k}\left(y\left(t_{k}^{i}\right)\right)
$$

Por (3.37), (3.21) e pelo fato de que $x$ é uma solução de (3.20), temos

$$
y(v)-y\left(t_{0}\right)=x(v)(v)-x\left(t_{0}\right)\left(t_{0}\right)=x(v)(v)-x\left(t_{0}\right)(v)=
$$




$$
=\left[\int_{t_{0}}^{v} D G(x(\tau), t)\right](v)=\left[\int_{t_{0}}^{v} D F(x(\tau), t)\right](v)+\left[\int_{t_{0}}^{v} D J(x(\tau), t)\right](v) .
$$

para $v \in\left[t_{0}, t_{0}+\sigma\right]$.

Usando (3.42) e (3.41), obtemos

$$
\begin{gathered}
y(v)-y\left(t_{0}\right)-\int_{t_{0}}^{v} f\left(y_{s}, s\right) d s-\sum_{k=0}^{j} \sum_{i=1}^{m\left(\tau_{k}\right)} I_{k}\left(y\left(t_{k}^{i}\right)\right) H_{k}^{i}(v)= \\
=\left[\int_{t_{0}}^{v} D F(x(\tau), t)\right](v)+\left[\int_{t_{0}}^{v} D J(x(\tau), t)\right](v)-\int_{t_{0}}^{v} f\left(y_{s}, s\right) d s-\sum_{k=1}^{j} \sum_{i=1}^{m\left(\tau_{k}\right)} I_{k}\left(y\left(t_{k}^{i}\right)\right) H_{k}^{i}(v)= \\
=\left[\int_{t_{0}}^{v} D F(x(\tau), t)\right](v)-\int_{t_{0}}^{v} f\left(y_{s}, s\right) d s .
\end{gathered}
$$

A existência das integrais $\int_{t_{0}}^{v} D G(x(\tau), t)$ e $\int_{t_{0}}^{v} D J(x(\tau), t)$ implica a existência da integral $\int_{t_{0}}^{v} D F(x(\tau), t)$.

Seja $\varepsilon>0$. Suponhamos que um calibre $\delta(\tau)>0$ de $\left[t_{0}, t_{0}+\sigma\right]$ satisfaça (3.39), (3.40) e as desigualdades

$$
|h(\rho)-h(\tau)|<\varepsilon, \text { para todo } \rho \in[\tau-\delta(\tau), \tau]
$$

$\mathrm{e}$

$$
|h(\rho)-h(\tau+)|<\varepsilon, \text { para todo } \rho \in(\tau, \tau+\delta(\tau)],
$$

onde $h(t)=h_{1}(t)+h_{2}(t)$ é a função não decrescente e contínua à esquerda descrita por (3.18) e (3.19).

Consideremos, também, o calibre $\delta$ de modo que se $\tau \in\left[t_{0}, t_{0}+\sigma\right]$, então

$$
\left|\int_{u}^{v} L(s) d s\right|<\frac{\varepsilon}{\left(\left(1+m\left(\tau_{1}\right)+\ldots+m\left(\tau_{j}\right)\right)+1\right)\left(K_{1}+1\right)}, \text { para todo }[u, v] \subset[\tau-\delta(\tau), \tau+\delta(\tau)]
$$

onde $\left(1+m\left(\tau_{1}\right)+\ldots+m\left(\tau_{j}\right)\right)$ é o número de momentos de impulso no intervalo $\left[t_{0}, t_{0}+\sigma\right]$ (se $t_{0}$ for um momento de impulso do sistema (3.1)-(3.2), como na demonstração do teorema anterior) e $K_{1}$ é a constante da condição $\left(A^{\prime}\right)$. Tal escolha é possível, pois a função $L:\left[t_{0}, t_{0}+\sigma\right] \rightarrow \mathbb{R}$ da condição $(B)$ é Lebesgue integrável.

Para o calibre $\delta$ que acabamos de definir e pela existência da integral $\int_{t_{0}}^{v} D F(x(\tau), t)$, temos a desigualdade

$$
\left\|\int_{t_{0}}^{v} D F(x(\tau), t)-\sum_{n}\left[F\left(x\left(\xi_{n}\right), s_{n}\right)-F\left(x\left(\xi_{n}\right), s_{n-1}\right)\right]\right\|<\varepsilon
$$


para toda partição $\delta$-fina $\left(\xi_{n},\left[s_{n-1}, s_{n}\right]\right)$ do intervalo $\left[t_{0}, v\right]$. Portanto

$$
\left|\int_{t_{0}}^{v} D F(x(\tau), t)(v)-\sum_{n}\left[F\left(x\left(\xi_{n}\right), s_{n}\right)-F\left(x\left(\xi_{n}\right), s_{n-1}\right)\right](v)\right|<\varepsilon
$$

para toda partição $\delta$-fina $\left(\xi_{n},\left[s_{n-1}, s_{n}\right]\right)$ do intervalo $\left[t_{0}, v\right]$. Por (3.43) e (3.48), temos

$$
\begin{gathered}
\left|y(v)-y\left(t_{0}\right)-\int_{t_{0}}^{v} f\left(y_{s}, s\right) d s-\sum_{k=0}^{j} \sum_{i=1}^{m\left(\tau_{k}\right)} I_{k}\left(y\left(t_{k}^{i}\right)\right) H_{k}^{i}(v)\right|= \\
=\left|\left(\int_{t_{0}}^{v} D F(x(\tau), t)\right)(v)-\int_{t_{0}}^{v} f\left(y_{s}, s\right) d s\right|< \\
\left.<\varepsilon+\mid \sum_{n}\left[F\left(x\left(\xi_{n}\right), s_{n}\right)-F\left(x\left(\xi_{n}\right), s_{n-1}\right)\right]\right](v)-\int_{t_{0}}^{v} f\left(y_{s}, s\right) d s \mid= \\
=\varepsilon+\left|\sum_{n}\left\{\left[F\left(x\left(\xi_{n}\right), s_{n}\right)-F\left(x\left(\xi_{n}\right), s_{n-1}\right)\right](v)-\int_{s_{n-1}}^{s_{n}} f\left(y_{s}, s\right) d s\right\}\right| .
\end{gathered}
$$

A definição de $F$ dada em (3.3) implica

$$
\left[F\left(x\left(\xi_{n}\right), s_{n}\right)-F\left(x\left(\xi_{n}\right), s_{n-1}\right)\right](v)=\int_{s_{n-1}}^{s_{n}} f\left(x\left(\xi_{n}\right)_{s}, s\right) d s
$$

Por (3.22), temos $x\left(\xi_{n}\right)(\vartheta)=x(\vartheta)(\vartheta)=y(\vartheta)$, para $\vartheta \leq \xi_{n}$. Portanto

$$
\begin{aligned}
\int_{s_{n-1}}^{s_{n}} f\left(x\left(\xi_{n}\right)_{s}, s\right) d s & -\int_{s_{n-1}}^{s_{n}} f\left(y_{s}, s\right) d s=\int_{s_{n-1}}^{s_{n}}\left[f\left(x\left(\xi_{n}\right)_{s}, s\right)-f\left(y_{s}, s\right)\right] d s= \\
& =\int_{\xi_{n}}^{s_{n}}\left[f\left(x\left(\xi_{n}\right)_{s}, s\right)-f\left(y_{s}, s\right)\right] d s .
\end{aligned}
$$

Analogamente, para $\vartheta \in\left[\xi_{n}, s_{n}\right]$, temos, novamente por (3.22), a igualdade $y(\vartheta)=x(\vartheta)(\vartheta)=x\left(s_{n}\right)(\vartheta)$ e portanto

$$
\int_{\xi_{n}}^{s_{n}}\left[f\left(x\left(\xi_{n}\right)_{s}, s\right)-f\left(y_{s}, s\right)\right] d s=\int_{\xi_{n}}^{s_{n}}\left[f\left(x\left(\xi_{n}\right)_{s}, s\right)-f\left(x\left(s_{n}\right)_{s}, s\right)\right] d s
$$

Usando as relações acima e a condição $(B)$, obtemos

$$
\begin{gathered}
\left|\left[F\left(x\left(\xi_{n}\right), s_{n}\right)-F\left(x\left(\xi_{n}\right), s_{n-1}\right)\right](v)-\int_{s_{n-1}}^{s_{n}} f\left(y_{s}, s\right) d s\right|= \\
=\left|\int_{\xi_{n}}^{s_{n}}\left[f\left(x\left(\xi_{n}\right)_{s}, s\right)-f\left(x\left(s_{n}\right)_{s}, s\right)\right] d s\right| \leq \int_{\xi_{n}}^{s_{n}} L(s)\left\|x\left(\xi_{n}\right)_{s}-x\left(s_{n}\right)_{s}\right\| d s .
\end{gathered}
$$


Agora, vamos analisar $\left\|x\left(\xi_{n}\right)_{s}-x\left(s_{n}\right)_{s}\right\|$. Pelo Lema 1.18 e pelo fato de que $x\left(s_{n}\right)\left(\xi_{n}\right)=x\left(\xi_{n}\right)\left(\xi_{n}\right)$ (veja (3.22)), temos para todo $n$ a seguinte relação:

$$
\begin{gathered}
\left\|x\left(\xi_{n}\right)_{s}-x\left(s_{n}\right)_{s}\right\|=\sup _{\vartheta \in[-r, 0]}\left|x\left(s_{n}\right)(s+\vartheta)-x\left(\xi_{n}\right)(s+\vartheta)\right|= \\
=\sup _{\rho \in\left[\xi_{n}, s_{n}\right]}\left|x\left(s_{n}\right)(\rho)-x\left(\xi_{n}\right)(\rho)\right|=\sup _{\rho \in\left(\xi_{n}, s_{n}\right]}\left|x\left(s_{n}\right)(\rho)-x\left(\xi_{n}\right)(\rho)\right|= \\
=\sup _{\rho \in\left(\xi_{n}, s_{n}\right]}\left|x\left(s_{n}\right)(\rho)-x\left(\xi_{n}+\right)(\rho)+x\left(\xi_{n}+\right)(\rho)-x\left(\xi_{n}\right)(\rho)\right| \leq \\
\leq \sup _{\rho \in\left(\xi_{n}, s_{n}\right]}\left\{\left|x\left(s_{n}\right)(\rho)-x\left(\xi_{n}+\right)(\rho)\right|+\left|x\left(\xi_{n}+\right)(\rho)-x\left(\xi_{n}\right)(\rho)\right|\right\} \leq \\
\leq\left\|x\left(s_{n}\right)-x\left(\xi_{n}+\right)\right\|+\left\|G\left(x\left(\xi_{n}\right), \xi_{n}+\right)-G\left(x\left(\xi_{n}\right), \xi_{n}\right)\right\| \leq h\left(s_{n}\right)-h\left(\xi_{n}+\right)+K_{1}<\varepsilon+K_{1},
\end{gathered}
$$

onde as últimas desigualdades decorrem do Lema 1.16 e da definição da $G$ em (3.17). Logo por (3.50), para todo $n$, a desigualdade

$$
\begin{gathered}
\left|\left[F\left(x\left(\xi_{n}\right), s_{n}\right)-F\left(x\left(\xi_{n}\right), s_{n-1}\right)\right](v)-\int_{s_{n-1}}^{s_{n}} f\left(y_{s}, s\right) d s\right| \leq \\
\leq \int_{\xi_{n}}^{s_{n}} L(s)\left\|x\left(\xi_{n}\right)_{s}-x\left(s_{n}\right)_{s}\right\| d s<\left(\varepsilon+K_{1}\right) \int_{\xi_{n}}^{s_{n}} L(s) d s
\end{gathered}
$$

é válida.

Agora, por (3.49) e (3.51), obtemos

$$
\begin{gathered}
\left|y(v)-y\left(t_{0}\right)-\int_{t_{0}}^{v} f\left(y_{s}, s\right) d s-\sum_{k=0}^{j} \sum_{i=1}^{m\left(\tau_{k}\right)} I_{k}\left(y\left(t_{k}^{i}\right)\right) H_{k}^{i}(v)\right|< \\
<\varepsilon+\sum_{n}\left|\left[F\left(x\left(\xi_{n}\right), s_{n}\right)-F\left(x\left(\xi_{n}\right), s_{n-1}\right)\right](v)-\int_{s_{n-1}}^{s_{n}} f\left(y_{s}, s\right) d s\right|< \\
<\varepsilon+\left(\varepsilon+K_{1}\right) \sum_{n} \int_{\xi_{n}}^{s_{n}} L(s) d s \leq \int_{t_{0}}^{s_{n}} L(s) d s \leq \\
\leq \varepsilon+\varepsilon \int_{t_{0}}^{v} L(s) d s+K_{1} \sum_{\left.n ; s_{n-1}, s_{n}\right) \cap\left\{t_{0}, t_{1}^{1}, \ldots, t_{1}^{m\left(\tau_{1}\right)}, \ldots, t_{j}, \ldots, t_{j}\left(\tau_{j}\right)\right\} \neq \emptyset} \int_{\xi_{n}}^{v} \leq \varepsilon\left(1+\int_{t_{0}}^{v} L(s) d s\right)+\varepsilon
\end{gathered}
$$


pela propriedade do calibre (3.46). Daí, tomando $\varepsilon>0$ de modo que

$$
\varepsilon\left(2+\int_{t_{0}}^{v} L(s) d s\right)<\eta
$$

obtemos (3.38) e a prova está completa.

Agora, consideremos a EDO generalizada (3.20). Sejam $t^{*} \in\left[t_{0},+\infty\right)$ e $\phi \in G^{-}\left([-r, 0], \mathbb{R}^{n}\right)$ uma função. Definamos uma função $\widetilde{x} \in P C_{1}$ por

$$
\widetilde{x}(\vartheta)=\left\{\begin{array}{l}
\phi\left(\vartheta-t_{0}\right), \text { para } \vartheta \in\left[t_{0}-r, t_{0}\right], \\
\phi(0), \text { para } \vartheta \in\left[t_{0},+\infty\right)
\end{array}\right.
$$

Considerando o problema de valor inicial (3.20)-(3.53), o Teorema 1.23 juntamente com os Teoremas 3.5 e 3.6 pode ser usado para obter o resultado seguinte.

Teorema 3.7. Se as condições $(C 1)$ até $(C 5)$ e $(A),(B),\left(A^{\prime}\right)$ e $\left(B^{\prime}\right)$ estiverem satisfeitas e se $\widetilde{x} \in P C_{1}$ de (3.53) for tal que

$$
\widetilde{x}(\vartheta)+H_{t_{l}}(\vartheta) I_{l}\left(\widetilde{x}\left(t^{*}\right)\right) \in P C_{1},
$$

quando $t^{*}=t_{l}^{i}$ para algum $l=0,1,2, \ldots$ e para algum $i=1, \ldots, m\left(\tau_{l}\right)$, então existirá um $\Delta>0$ tal que, no intervalo $\left[t^{*}, t^{*}+\Delta\right]$, existirá uma única solução y: $\left[t^{*}, t^{*}+\Delta\right] \rightarrow \mathbb{R}^{n}$ da EDFR impulsiva (3.1) que satisfará $y_{t^{*}}=\phi$.

Pelo Teorema 1.23, para $\widetilde{x} \in P C_{1}$ a relação

$$
\widetilde{x}_{+}=\widetilde{x}+G\left(\widetilde{x}, t^{*}+\right)-G\left(\widetilde{x}, t^{*}\right) \in P C_{1}
$$

é necessária. Tal condição garante que a solução do problema de valor inicial (3.20)-(3.53) não deixe de pertencer ao conjunto $P C_{1}$ imediatamente no momento $t^{*}$. Note que, em nossa situação, para a função $G$ dada por (3.20), temos

$$
G\left(\widetilde{x}, t^{*}+\right)-G\left(\widetilde{x}, t^{*}\right)=0 \quad \text { se } \quad t^{*} \neq t_{l}^{i}, l=0,1,2, \ldots ; i=1, \ldots, m\left(\tau_{l}\right)
$$

$\mathrm{e}$

$$
\left[G\left(\widetilde{x}, t^{*}+\right)-G\left(\widetilde{x}, t^{*}\right)\right](\vartheta)=H_{t_{l}}(\vartheta) I_{l}\left(\widetilde{x}\left(t^{*}\right)\right) \quad \text { se } \quad t^{*}=t_{l}^{i}, l=0,1,2, \ldots ; i=1, \ldots, m\left(\tau_{l}\right)
$$


e (3.55) nos dá a condição (3.54) do Teorema 3.7.

O Teorema 3.7 sobre existência e unicidade para o sistema impulsivo (3.1)-(3.2) será imprescindível para o próximo capítulo.

Pelos Teoremas 3.5 e 3.6, temos uma correspondência biunívoca entre a solução do problema de valor inicial (3.1)-(3.2) e a solução do problema de valor inicial (3.20)-(3.53). Considerando o Teorema 3.7, o conceito de solução maximal para o problema (3.20)-(3.53) pode ser introduzido tomando-se $\sigma=$ $\sup \Delta>0$, onde $\Delta>0$ é tal que existe uma única solução $y:\left[t_{0}, t_{0}+\Delta\right] \rightarrow \mathbb{R}^{n}$ da equação (3.1) que satisfaz $y_{t_{0}}=\phi$. Dessa forma, a função $y:\left[t_{0}, t_{0}+\sigma\right) \rightarrow \mathbb{R}^{n}$ é a solução maximal do problema de valor inicial (3.1)-(3.2), ou seja, $y$ é solução de (3.1)-(3.2) em todo intervalo fechado $\left[t_{0}, t_{0}+\Delta\right] \operatorname{com} 0<\Delta<\sigma$, mas não é solução de (3.1)-(3.2) nos intervalos $\left[t_{0}, t_{0}+\Delta\right] \operatorname{com} \Delta \geq \sigma$. 
CAPÍTULO

4

\section{Propriedades qualitativas de}

\section{soluções de EDFRs impulsivas via}

\section{EDOs generalizadas}

Neste capítulo, vamos considerar a EDFR com ação impulsiva

$$
\begin{cases}\dot{y}(t)=f\left(y_{t}, t\right), & t \neq \tau_{k}(y(t)), \quad t \geq t_{0}, \\ \Delta y(t)=I_{k}(y(t)), & t=\tau_{k}(y(t)), \quad k=0,2, \ldots,\end{cases}
$$

com condição inicial

$$
y_{t_{0}}=\phi
$$

onde $f: G^{-}\left([-r, 0], \mathbb{R}^{n}\right) \times\left[t_{0},+\infty\right) \rightarrow \mathbb{R}^{n}, t_{0} \geq 0$ e $\phi \in G^{-}\left([-r, 0], \mathbb{R}^{n}\right)$. Consideraremos que, para cada $y \in G^{-}\left(\left[t_{0}-r,+\infty\right), \mathbb{R}^{n}\right)$, a aplicação $t \mapsto f\left(t, y_{t}\right)$ seja localmente Lebesgue integrável, para $t \geq t_{0}$. Vamos considerar, também, que os operadores de impulso $I_{k}(x), k=0,1,2, \ldots$, sejam funções contínuas de $\mathbb{R}^{n}$ em $\mathbb{R}^{n}$ e que

$$
\Delta y(t)=y(t+)-y(t-)=y(t+)-y(t)=I_{k}(y(t)), \quad k=0,1,2, \ldots,
$$

para cada $y \in G^{-}\left(\left[t_{0}-r,+\infty\right), \mathbb{R}^{n}\right)$ e para cada $t \geq t_{0}$

Consideraremos $I_{0} \equiv 0$, se $t_{0}$ não for um momento de impulso do sistema (4.1)-(4.2). Além disso, suporemos que as condições $(C 1)$ até $(C 5)$ e $(A),(B),\left(A^{\prime}\right)$ e $\left(B^{\prime}\right)$ do Capítulo 3 estejam satisfeitas.

Vamos investigar algumas propriedades qualitativas de soluções de (4.1) através da teoria das EDOs generalizadas, usando funcionais de Lyapunov, os quais serão especificados a seguir. 
Dados $t \geq t_{0}$ e uma função $\psi \in G^{-}\left([-r, 0], \mathbb{R}^{n}\right)$, consideremos a EDFR com impulsos em tempo variável (4.1) com condição inicial $y_{t}=\psi$. Consideremos, também, a EDO generalizada (3.20) sujeita à condição inicial $x(t)=\widetilde{x}$, onde $\widetilde{x}(\tau)=\psi(\tau-t), t-r \leq \tau \leq t$, e $\widetilde{x}(\tau)=\psi(0), \tau \geq t$. Suponhamos que, $\widetilde{x}_{+}=\widetilde{x}+G(\widetilde{x}, t+)-G(\widetilde{x}, t) \in P C_{1}$. Pelo Teorema 3.7, existe uma única solução $y:[t-r, v] \rightarrow \mathbb{R}^{n}$ da EDFR impulsiva (4.1) que satisfaz $y_{t}=\psi$. E, pelo Teorema 3.5, podemos encontrar uma solução $x:[t, v] \rightarrow G^{-}\left([t, v], \mathbb{R}^{n}\right)$ da EDO generalizada (3.20), com condição inicial $x(t)=\widetilde{x}$. Então $x(t)(t+\theta)=$ $y(t+\theta)$ para todo $\theta \in[-r, 0]$ e, portanto, $(x(t))_{t}=y_{t}$. Neste caso, escrevemos $y_{t+\eta}=y_{t+\eta}(t, \psi)$ para todo $\eta \geq 0$. Então, para $U:\left[t_{0},+\infty\right) \times G^{-}\left([-r, 0], \mathbb{R}^{n}\right) \rightarrow \mathbb{R}$, definimos

$$
D^{+} U(t, \psi)=\limsup _{\eta \rightarrow 0+} \frac{U\left(t+\eta, y_{t+\eta}(t, \psi)\right)-U\left(t, y_{t}(t, \psi)\right)}{\eta}, \quad t \geq t_{0}
$$

Por outro lado, dado $t \geq t_{0}$, se $\widetilde{x} \in G^{-}\left([t-r,+\infty), \mathbb{R}^{n}\right)$ for tal que $\widetilde{x}(\tau)=\psi(\tau-t), t-r \leq \tau \leq t$, e $\widetilde{x}(\tau)=\psi(0), \tau \geq t$, então existirá, pelo Teorema 1.23, uma única solução $x:[t, \bar{v}] \rightarrow G^{-}\left([t, \bar{v}], \mathbb{R}^{n}\right)$ da EDO generalizada (3.20) tal que $x(t)=\widetilde{x}$, com $[t, \bar{v}] \subset\left[t_{0},+\infty\right)$. Pelo Teorema 3.6, podemos encontrar uma solução $y:[t-r, \bar{v}] \rightarrow \mathbb{R}^{n}$ de (4.1) que satisfaz $y_{t}=\psi$ e é descrita em termos de $x$. Neste caso, escrevemos $x_{\psi}(t)$ em lugar de $x(t)$ e temos $y_{t}(t, \psi)=\left(x_{\psi}(t)\right)_{t}=\psi$. Consequentemente, $\left(t, x_{\psi}(t)\right) \mapsto$ $\left(t, y_{t}(t, \psi)\right)$ é uma aplicação biunívoca e podemos definir uma função $V:\left[t_{0},+\infty\right) \times P C_{1} \rightarrow \mathbb{R}$ pondo

$$
V\left(t, x_{\psi}(t)\right)=U\left(t, y_{t}(t, \psi)\right)
$$

Portanto

$$
D^{+} U(t, \psi)=\limsup _{\eta \rightarrow 0^{+}} \frac{V\left(t+\eta, x_{\psi}(t+\eta)\right)-V\left(t, x_{\psi}(t)\right)}{\eta}, \quad t \geq t_{0} .
$$

Observação 4.1. Com a notação anterior, dado $t \geq t_{0}$, temos $\left\|y_{t}(t, \psi)\right\|=\left\|x_{\psi}(t)\right\|$, já que

$$
\begin{aligned}
\left\|y_{t}(t, \psi)\right\| & =\left\|y_{t}\right\|=\sup _{-r \leq \theta \leq 0}|y(t+\theta)|=\sup _{t-r \leq \tau \leq t}|y(\tau)|=\sup _{t-r \leq \tau \leq t}\left|x_{\psi}(t)(\tau)\right| \\
& =\sup _{t-r \leq \tau<+\infty}\left|x_{\psi}(t)(\tau)\right|=\left\|x_{\psi}(t)\right\|,
\end{aligned}
$$

onde usamos o Teorema 3.5 para obter a quarta igualdade.

A seguir, veremos quando $U:\left[t_{0},+\infty\right) \times G^{-}\left([-r, 0], \mathbb{R}^{n}\right) \rightarrow \mathbb{R}$ define um funcional de Lyapunov. Tendo como base a Definição 2.3, temos o conceito seguinte.

Definição 4.2. Diremos que $U:\left[t_{0},+\infty\right) \times G^{-}\left([-r, 0], \mathbb{R}^{n}\right) \rightarrow \mathbb{R}$ é um funcional de Lyapunov (com respeito a EDFR impulsiva (4.1)) se as seguintes condições forem satisfeitas: 
(i) $U(\cdot, \psi):\left[t_{0},+\infty\right) \rightarrow \mathbb{R}$ é contínuo à esquerda em $\left(t_{0},+\infty\right)$ para qualquer $\psi \in G^{-}\left([-r, 0], \mathbb{R}^{n}\right)$;

(ii) Existe uma função monótona crescente $b: \mathbb{R}_{+} \rightarrow \mathbb{R}_{+}$tal que $b(0)=0 e$

$$
U(t, \psi) \geq b(\|\psi\|)
$$

para cada $t \geq t_{0}$ e para cada $\psi \in G^{-}\left([-r, 0], \mathbb{R}^{n}\right)$

(iii) A desigualdade

$$
D^{+} U(t, \psi) \leq 0
$$

vale para cada $t \geq t_{0}$ e para cada $\psi \in G^{-}\left([-r, 0], \mathbb{R}^{n}\right)$.

Observação 4.3. Se $U$ for um funcional de Lyapunov, então o funcional $V$ dado pela relação (4.3) satisfará as condições (i), (ii) e (iii) da Definição 2.3, ou seja, V também será um funcional de Lyapunov. Por (4.3) e (4.4), verificamos facilmente que $V$ satisfaz as condições (i) e (iii) da Definição 2.3. Portanto basta constatarmos que $V$ satisfaz (ii). Com efeito, se $t \geq t_{0}$ e $z \in P C_{1}$, existirão uma solução $x$ da EDO generalizada (3.20) e uma função $\psi \in G^{-}\left([-r, 0], \mathbb{R}^{n}\right)$ tais que $z=x_{\psi}(t),\left(x_{\psi}(t)\right)_{t}=y_{t}(t, \psi)$, onde yé a solução da EDFR impulsiva (4.1) correspondente a x. Mas

$$
\|\psi\|=\left\|y_{t}(t, \psi)\right\|=\left\|x_{\psi}(t)\right\|=\|z\|
$$

pela Observação 4.1. Como U é um funcional de Lyapunov, pela condição (ii) da Definição 4.2, obtemos

$$
V(t, z)=V\left(t, x_{\psi}(t)\right)=U\left(t, y_{t}(t, \psi)\right)=U(t, \psi) \geq b(\|\psi\|)=b(\|z\|)
$$

Dividiremos o presente capítulo em três seções. Na primeira seção, estabeleceremos resultados de estabilidade uniforme e estabilidade uniforme assintótica da solução nula da EDFR impulsiva (4.1), enquanto que, na segunda seção, apresentaremos um resultado de estabilidade exponencial para a solução nula da EDFR impulsiva (4.1). Na terceira seção, obteremos resultados de limitação uniforme para a solução do problema de valor inicial (4.1)-(4.2).

As principais referências para este capítulo são os nossos trabalhos [1], [2] e [3]. 


\subsection{Estabilidade da solução trivial}

Nesta seção, suporemos que

$$
f(0, t)=0 \text { para todo } t \geq t_{0} \text { e } I_{k}(0)=0, k=0,1,2, \ldots
$$

Isso implicará que a função $y \equiv 0$ é uma solução da EDFR impulsiva (4.1) em qualquer intervalo contido em $\left[t_{0},+\infty\right)$. Também consideraremos o conjunto $E_{c}=\left\{\psi \in G^{-}\left([-r, 0], \mathbb{R}^{n}\right):\|\psi\|<c\right\}, c>0$.

A seguir, apresentaremos alguns conceitos básicos de estabilidade.

Definição 4.4. A solução trivial $y \equiv 0$ de (4.1) será dita

(i) Estável (no sentido de Lyapunov), se para quaisquer $t_{0} \geq 0$ e $\varepsilon>0$, existir $\delta=\delta\left(\varepsilon, t_{0}\right)>0$ tal que se $\phi \in E_{c}$ e $\bar{y}:\left[t_{0}-r, v\right] \rightarrow \mathbb{R}^{n}$ for uma solução de (4.1) em $\left[t_{0}, v\right]$ satisfazendo a condição inicial $\bar{y}_{t_{0}}=\phi e$

$$
\|\phi\|<\delta
$$

então

$$
\left\|\bar{y}_{t}\left(t_{0}, \phi\right)\right\|<\varepsilon, \quad t \in\left[t_{0}, v\right] .
$$

(ii) Uniformemente estável, se o número $\delta$ no item (i) for independente de $t_{0}$.

(iii) Uniformemente assintoticamente estável, se existir $\delta_{0}>0$ e para todo $\varepsilon>0$, existir $T=T(\varepsilon) \geq 0$ tal que se $\phi \in E_{c}$ e $\bar{y}:\left[t_{0}-r, v\right] \rightarrow \mathbb{R}^{n}$ for solução de (4.1) em $\left[t_{0}, v\right]$ satisfazendo a condição inicial $\bar{y}_{t_{0}}=\phi e$

$$
\|\phi\|<\delta_{0}
$$

então

$$
\left\|\bar{y}_{t}\left(t_{0}, \phi\right)\right\|<\varepsilon, \quad t \in\left[t_{0}, v\right] \cap\left[t_{0}+T,+\infty\right) .
$$

No que segue, consideraremos os conjuntos $\bar{E}_{\rho}=\left\{y \in G^{-}\left([-r, 0], \mathbb{R}^{n}\right):\|y\| \leq \rho\right\}$ e $\bar{B}_{\rho}=\left\{x \in P C_{1}\right.$ : $\|x\| \leq \rho\}, \operatorname{com} 0<\rho<c$.

Veremos que a função $V$ dada por (4.3) satisfará algumas propriedades se a função $U$ também satisfizer algumas propriedades.

Lema 4.5 ([2], Lema 4.4). Considere a EDFR impulsiva (4.1). Suponha que a função $U:\left[t_{0},+\infty\right) \times$ $\bar{E}_{\rho} \rightarrow \mathbb{R}$ satisfaça as seguintes condições: 
(i) $U(t, 0)=0, t \in\left[t_{0},+\infty\right)$;

(ii) Existe uma constante $K>0$ tal que

$$
|U(t, \psi)-U(t, \bar{\psi})| \leq K\|\psi-\bar{\psi}\|, \quad t \in\left[t_{0},+\infty\right), \psi, \bar{\psi} \in \bar{E}_{\rho}
$$

Então a função $V:\left[t_{0},+\infty\right) \times \bar{B}_{\rho} \rightarrow \mathbb{R}$ definida por (4.3) satisfaz $V(t, 0)=0$ para todo $t \in\left[t_{0},+\infty\right)$, e

$$
|V(t, z)-V(t, \bar{z})| \leq K\|z-\bar{z}\|
$$

para $t \geq t_{0}$ e $z, \bar{z} \in \bar{B}_{\rho}$

Demonstração. Dados $t \geq t_{0}$ e $\psi, \bar{\psi} \in \bar{E}_{\rho}$, sejam $y, \bar{y}, \widehat{y}:[t-r,+\infty) \rightarrow \mathbb{R}^{n}$ soluções da EDFR impulsiva (4.1) tal que $y_{t}=\psi, \bar{y}_{t}=\bar{\psi}$ e $\widehat{y}_{t}=0$. Sejam $x, \bar{x}, \widehat{x}$ soluções em $[t,+\infty)$ da EDO generalizada (3.20) dadas pelo Teorema 3.5 correspondentes a $y, \bar{y}$ e $\widehat{y}$, respectivamente. Então $(x(t))_{t}=y_{t}=\psi,(\bar{x}(t))_{t}=\bar{y}_{t}=\bar{\psi} \mathrm{e}$ $(\widehat{x}(t))_{t}=\widehat{y}_{t}=0$. Pela Observação 4.1, $x_{\psi}(t), x_{\bar{\psi}}(t) \in \bar{B}_{\rho}$.

Como $f$ satisfaz $(A)$ e $(B)$ e $I_{k}$ satisfaz $\left(A^{\prime}\right)$ e $\left(B^{\prime}\right)$ para $k=0,1,2, \ldots$, então a função $G$ na equação (3.20) pertence à classe $\mathscr{F}(\Omega, h)$, onde $\Omega=P C_{1} \times[t,+\infty)$ e $h=h_{1}+h_{2}$, com $h_{1}$ dada por (3.7) e $h_{2}$ dada por (3.14).

Seja $V:\left[t_{0},+\infty\right) \times \bar{B}_{\rho} \rightarrow \mathbb{R}$ dada por (4.3). Pela condição $(i)$, temos

$$
0=U(t, 0)=U\left(t, \widehat{y}_{t}(t, 0)\right)=V(t, \widehat{x}(t))=V(t, 0),
$$

já que $\widehat{x}(t)(\tau)=0$ para todo $\tau$ (veja o Teorema 3.5), isto é, $\widehat{x}(t) \equiv 0$.

Pela condição $(i i)$, temos

$$
\left|V\left(t, x_{\psi}(t)\right)-V\left(t, \bar{x}_{\bar{\psi}}(t)\right)\right|=\left|U\left(t, y_{t}(t, \psi)\right)-U\left(t, \bar{y}_{t}(t, \bar{\psi})\right)\right|=|U(t, \psi)-U(t, \bar{\psi})|
$$

Então, pela Observação 4.1, obtemos

$$
\left|V\left(t, x_{\psi}(t)\right)-V\left(t, \bar{x}_{\bar{\psi}}(t)\right)\right| \leq K\|\psi-\bar{\psi}\|=K\left\|x_{\psi}(t)-\bar{x}_{\bar{\psi}}(t)\right\|
$$

É claro que, dados $t \geq t_{0}$ e funções $z, \bar{z} \in \bar{B}_{\rho}$, existem soluções $x$ e $\bar{x}$ da EDO generalizada (3.20) e $\psi, \bar{\psi} \in G^{-}\left([-r, 0], \mathbb{R}^{n}\right)$ tais que $z=x_{\psi}(t),\left(x_{\psi}(t)\right)_{t}=y_{t}(t, \psi), \bar{z}=\bar{x}_{\bar{\psi}}(t)$ e $\left(\bar{x}_{\bar{\psi}}(t)\right)_{t}=\bar{y}_{t}(t, \bar{\psi})$. 
Note que

$$
\|\psi\|=\left\|y_{t}(t, \psi)\right\|=\left\|x_{\psi}(t)\right\|=\|z\| \leq \rho
$$

$\mathrm{e}$

$$
\|\bar{\psi}\|=\left\|\bar{y}_{t}(t, \bar{\psi})\right\|=\left\|\bar{x}_{\bar{\psi}}(t)\right\|=\|\bar{z}\| \leq \rho
$$

pela Observação 4.1. Portanto, por (4.5), temos

$$
|V(t, z)-V(t, \bar{z})| \leq K\|z-\bar{z}\|
$$

e o resultado segue.

Observação 4.6. Os dois próximos resultados, a saber o Teorema 4.7 e o Teorema 4.8, são teoremas do tipo Lyapunov para a EDFR impulsiva (4.1) e são análogos aos Teoremas 4.8 e 4.9 de [10], respectivamente. Tais resultados de [10] tratam de estabilidade uniforme e estabilidade uniforme assintótica para uma classe de EDFRs com impulsos em tempo pré-fixado. Nossos resultados tratam de estabilidade uniforme e estabilidade uniforme assintótica para uma classe de EDFRs com impulsos em tempo variável. É importante observar que, em [10], os autores consideram um funcional $U:[0,+\infty) \times G^{-}\left([-r, 0], \mathbb{R}^{n}\right) \rightarrow$ $\mathbb{R}$ com respeito a EDFR impulsiva (4.1) e um funcional $V:[0,+\infty) \times G^{-}\left([-r,+\infty), \mathbb{R}^{n}\right) \rightarrow \mathbb{R}$ com respeito a EDO generalizada (3.20) e consideram que $U$ e $V$ estão relacionados pela igualdade

$$
V(t, x)=U\left(t, x_{t}\right)
$$

para $t \geq 0$ e $x \in G^{-}\left([-r,+\infty), \mathbb{R}^{n}\right)$. No presente trabalho, nós relacionamos $U$ e V pela igualdade (4.3). Também, em [10], foi suposto que

$$
U\left(t, y_{t}\right) \geq b(\|y\|), \quad t \in[0,+\infty), y \in \bar{B}_{\rho}
$$

onde b é uma função monótona crescente que satisfaz $b(0)=0$. Aqui, substituiremos a condição (4.6) pela condição mais fraca

$$
U(t, \psi) \geq b(\|\psi\|), \quad t \in\left[t_{0},+\infty\right), \psi \in \bar{E}_{\rho}
$$

onde $t_{0} \geq 0$. As provas dos Teoremas 4.7 e 4.8 foram realizadas seguindo as ideias das provas dos Teoremas 4.8 e 4.9 de [10], respectivamente.

Teorema 4.7 ([2], Teorema 4.6). Considere a EDFR impulsiva (4.1). Suponha que $U:\left[t_{0},+\infty\right) \times \bar{E}_{\rho} \rightarrow$ $\mathbb{R}$ seja um funcional de Lyapunov e que as seguintes condições sejam válidas: 
(i) $U(t, 0)=0, t \in\left[t_{0},+\infty\right)$;

(ii) Existe uma constante $K>0$ tal que

$$
|U(t, \psi)-U(t, \bar{\psi})| \leq K\|\psi-\bar{\psi}\|, \quad t \in\left[t_{0},+\infty\right), \quad \psi, \bar{\psi} \in \bar{E}_{\rho}
$$

Então a solução trivial $y \equiv 0$ de (4.1) será uniformemente estável.

Demonstração. Como $f$ satisfaz as condições $(A)$ e $(B)$ e $I_{k}$ satisfaz $\left(A^{\prime}\right)$ e $\left(B^{\prime}\right)$ para $k=0,1,2, \ldots$, a função $G$ na equação (3.20) pertence à classe $\mathscr{F}(\Omega, h)$, onde $\Omega=P C_{1} \times\left[t_{0},+\infty\right)$ e $h=h_{1}+h_{2}$, com $h_{1}$ dada por (3.7) e $h_{2}$ dada por (3.14).

Seja $V:\left[t_{0},+\infty\right) \times \bar{B}_{\rho} \rightarrow \mathbb{R}$ dada por (4.3). Pelo Lema 4.5,

$$
V(t, 0)=0, \quad \text { para } t \in\left[t_{0},+\infty\right)
$$

e

$$
|V(t, z)-V(t, \bar{z})| \leq K\|z-\bar{z}\|, \quad \text { para } t \in\left[t_{0},+\infty\right) \quad \text { e } \quad z, \bar{z} \in \bar{B}_{\rho} .
$$

Pela Observação 4.3, $V$ é um funcional de Lyapunov. Portanto, as hipóteses do Teorema 2.6 estão satisfeitas. Então a solução $x \equiv 0$ da EDO generalizada (3.20) é variacionalmente estável. Logo para todo $\varepsilon>0$, existe $\delta=\delta(\varepsilon)>0$ tal que se $\bar{x}:[\gamma, v] \rightarrow B_{\rho}, 0 \leq t_{0}<v<+\infty$, for uma função de variação limitada em $\left[t_{0}, v\right]$ e contínua à esquerda em $\left(t_{0}, v\right]$ tal que

$$
\|\bar{x}(\gamma)\|<\delta
$$

e

$$
\operatorname{var}_{t_{0}}^{v}\left(\bar{x}(s)-\int_{\gamma}^{s} D G(\bar{x}(\tau), t)\right)<\delta
$$

então

$$
\|\bar{x}(t)\|<\varepsilon, \quad t \in\left[t_{0}, v\right] .
$$

Sejam $\phi \in \bar{E}_{\rho}$ e $\bar{y}:\left[t_{0}-r,+\infty\right) \rightarrow \mathbb{R}^{n}$ uma solução de (4.1) tal que $\bar{y}_{t_{0}}=\phi$. Suponhamos que

$$
\|\phi\|<\delta
$$


Queremos provar que

$$
\left\|\bar{y}_{t}\left(t_{0}, \phi\right)\right\|<\varepsilon, \quad t \in\left[t_{0},+\infty\right) .
$$

Vamos denotar $\bar{y}_{t}=\bar{y}_{t}\left(t_{0}, \phi\right)$ e definir

$$
\bar{x}(t)(\tau)=\left\{\begin{array}{l}
\bar{y}(\tau), t_{0}-r \leq \tau \leq t \\
\bar{y}(t), \tau \geq t
\end{array}\right.
$$

Pelo Teorema 3.5, $\bar{x}(t)$ é uma solução em $\left[t_{0},+\infty\right)$ da EDO generalizada (3.20) satisfazendo a condição inicial $\bar{x}\left(t_{0}\right)=\widetilde{x}$, onde

$$
\widetilde{x}(\tau)= \begin{cases}\phi\left(\tau-t_{0}\right), & t_{0}-r \leq \tau \leq t_{0} \\ \phi(0), & \tau \geq t_{0}\end{cases}
$$

Além disso, $\bar{x}$ é contínua à esquerda, pelo Lema 1.16 e de variação limitada em $\left[t_{0},+\infty\right)$, pelo Corolário 1.17.

Por (4.12) e (4.9), temos

$$
\left\|\bar{x}\left(t_{0}\right)\right\|=\sup _{t_{0}-r \leq \tau<+\infty}|\widetilde{x}(\tau)|=\|\phi\|<\delta
$$

E mais, para todo $v \in\left(t_{0},+\infty\right)$, temos

$$
\operatorname{var}_{t_{0}}^{v}\left(\bar{x}(s)-\int_{t_{0}}^{s} D G(\bar{x}(\tau), t)\right)=0<\delta
$$

pela Observação 2.2. Por conseguinte, (4.8) vale, isto é, $\|\bar{x}(t)\|<\varepsilon$ para todo $t \in\left[t_{0}, v\right]$. Em particular, $\|\bar{x}(v)\|<\varepsilon$. Então (4.11) implica que, para qualquer $t \in\left[t_{0}, v\right]$,

$$
\begin{aligned}
\left\|\bar{y}_{t}\left(t_{0}, \phi\right)\right\| & =\left\|\bar{y}_{t}\right\|=\sup _{-r \leq \theta \leq 0}|\bar{y}(t+\theta)| \leq \sup _{t_{0}-r \leq \tau \leq v}|\bar{y}(\tau)| \\
& =\sup _{t_{0}-r \leq \tau \leq v}|\bar{x}(v)(\tau)|=\sup _{t_{0}-r \leq \tau<+\infty}|\bar{x}(v)(\tau)| \\
& =\|\bar{x}(v)\|<\varepsilon .
\end{aligned}
$$

Como vé arbitrário, (4.10) vale e a prova está completa.

Teorema 4.8 ([2], Teorema 4.7). Considere a EDFR impulsiva (4.1). Suponha que $U:\left[t_{0},+\infty\right) \times \overline{E_{\rho}} \rightarrow \mathbb{R}$ seja um funcional de Lyapunov e que satisfaça as condições $(i)$ e (ii) do Teorema 4.7. Além disso, suponha que exista uma função contínua $\Lambda: \mathbb{R}_{+} \rightarrow \mathbb{R}_{+}$tal que $\Lambda(0)=0$ e $\Lambda(x)>0$ se $x \neq 0$, de forma 
que, para qualquer $\psi \in \bar{E}_{\rho}$, tenhamos

$$
D^{+} U(t, \psi) \leq-\Lambda(\|\psi\|), \quad t \geq t_{0}
$$

Então a solução trivial $y \equiv 0$ de (4.1) será uniformemente assintoticamente estável.

\section{Demonstração.}

Seja $V:\left[t_{0},+\infty\right) \times \bar{B}_{\rho} \rightarrow \mathbb{R}$ dada por (4.3). Então as hipóteses do Teorema 2.6 estão satisfeitas.

Definamos $\Phi: \bar{B}_{\rho} \rightarrow \mathbb{R}$ por $\Phi(z)=\Lambda(\|z\|)$, para $z \in \bar{B}_{\rho}$. Então $\Phi$ é contínua, $\Phi(0)=0$ e $\Phi(z)>0$ sempre que $z \neq 0$.

Suponhamos que $x:[t,+\infty) \rightarrow \bar{B}_{\rho}$ seja uma solução de (3.20) tal que $(x(t))_{t}=\psi$, onde $t \in\left[t_{0},+\infty\right)$ e $\psi \in \bar{E}_{\rho}$, e consideremos $y:[t-r,+\infty) \rightarrow \mathbb{R}^{n}$ a solução de (4.1), dada pelo Teorema 3.5 , que satisfaz $y_{t}=\psi$. Por (4.16), temos

$$
\begin{gathered}
\limsup _{\eta \rightarrow 0+} \frac{V\left(t+\eta, x_{\psi}(t+\eta)\right)-V\left(t, x_{\psi}(t)\right)}{\eta}=D^{+} U\left(t, y_{t}(t, \psi)\right)= \\
=D^{+} U(t, \psi) \leq-\Lambda(\|\psi\|)=-\Lambda\left(\left\|y_{t}\right\|\right) .
\end{gathered}
$$

Todavia,

$$
\left\|y_{t}\right\|=\left\|x_{\psi}(t)\right\|
$$

pela Observação 4.1. Portanto,

$$
\limsup _{\eta \rightarrow 0+} \frac{V\left(t+\eta, x_{\psi}(t+\eta)\right)-V\left(t, x_{\psi}(t)\right)}{\eta} \leq-\Lambda\left(\left\|y_{t}\right\|\right)=-\Lambda\left(\left\|x_{\psi}(t)\right\|\right)=-\Phi\left(x_{\psi}(t)\right)
$$

e as hipóteses do Teorema 2.7 estão satisfeitas. Então $x \equiv 0$ é variacionalmente assintoticamente estável, ou seja, existe $\delta_{0}>0$ e para todo $\varepsilon>0$, existem $T=T(\varepsilon) \geq 0$ e $\rho=\rho(\varepsilon)>0$ tal que se $\bar{x}:[\gamma, \nu] \rightarrow B_{c}$, $t_{0} \leq \gamma<v<+\infty$, for uma função de variação limitada em $[\gamma, v]$ e contínua à esquerda em $(\gamma, v]$ tal que

$$
\|\bar{x}(\gamma)\|<\delta_{0}
$$

$\mathrm{e}$

$$
\operatorname{var}_{\gamma}^{v}\left(\bar{x}(s)-\int_{\gamma}^{s} D G(\bar{x}(\tau), t)\right)<\rho
$$


então

$$
\|\bar{x}(t)\|<\varepsilon, \quad t \in[\gamma, v] \cap[\gamma+T,+\infty), \gamma \geq t_{0} .
$$

Dado $\varepsilon>0$, sejam $\delta_{0}>0$ e $T=T(\varepsilon)$ como acima. Seja $\phi \in E_{c}$, e consideremos $\bar{y}:\left[t_{0}-r,+\infty\right) \rightarrow \mathbb{R}^{n}$ a solução de (4.1) tal que $\bar{y}_{t_{0}}=\phi$ e

$$
\|\phi\|<\delta_{0} .
$$

Queremos provar que

$$
\left\|\bar{y}_{t}\left(t_{0}, \phi\right)\right\|<\varepsilon, \quad t \in\left[t_{0}+T,+\infty\right) .
$$

Mas isto segue imediatamente como na prova do Teorema 4.7. Por (4.20), obtemos (4.17) como em (4.13). Também, como no Teorema 4.7, temos (4.14) e, portanto, vale (4.18). Finalmente vale (4.21), uma vez que temos (4.15), como no Teorema 4.7, e (4.19).

Agora, consideraremos funções $U:\left[t_{0}-r,+\infty\right) \times \mathbb{R}^{n} \rightarrow \mathbb{R}$. Estabeleceremos novos resultados sobre estabilidade da solução trivial da EDFR impulsiva (4.1). Vamos definir a derivada de $U$ ao longo das soluções de (4.1) por

$$
D^{+} U(t, y(t))=\limsup _{\eta \rightarrow 0+} \frac{U(t+\eta, y(t+\eta, t, \psi))-U(t, y(t, t, \psi))}{\eta}, \quad t \geq t_{0},
$$

onde $y(s, t, \psi)$ é a solução de (4.1) que satisfaz $y_{t}=\psi$, com $\psi \in G^{-}\left([-r, 0], \mathbb{R}^{n}\right)$. Note que podemos escrever $D^{+} U(t, \psi(0))$ ao invés de $D^{+} U(t, y(t))$, visto que dados $t \geq t_{0}$ e uma função inicial $\psi \in G^{-}\left([-r, 0], \mathbb{R}^{n}\right)$, existe uma única solução de (4.1) que satisfaz $y_{t}=\psi$ (veja Teorema 3.7) e, portanto, $y(t)=\psi(0)$.

Teorema 4.9 ([2], Teorema 4.8). Considere a EDFR impulsiva (4.1). Suponha que $U:\left[t_{0}-r,+\infty\right) \times$ $\mathbb{R}^{n} \rightarrow \mathbb{R}$ seja contínua à esquerda em $\left(t_{0}-r,+\infty\right)$ e que os limites

$$
U(t-, y(t-))=\lim _{s \rightarrow t^{-}} U(s, y(s)), \quad t \in\left(t_{0}-r,+\infty\right)
$$

$e$

$$
U(t+, y(t+))=\lim _{s \rightarrow t^{+}} U(s, y(s)), \quad t \in\left[t_{0}-r,+\infty\right),
$$

existam, com a igualdade $U(t-, y(t-))=U(t, y(t))$ satisfeita, para $y \in G^{-}\left(\left[t_{0}-r,+\infty\right), \mathbb{R}^{n}\right)$. Além disso, suponha que $U$ satisfaça as seguintes condições:

(i) $U(t, 0)=0, t \in\left[t_{0}-r,+\infty\right)$; 
(ii) Para cada a $>0$, existe uma constante $K_{a}>0$ tal que

$$
|U(t, x)-U(t, y)| \leq K_{a}\|x-y\|, \quad t \in\left[t_{0}-r,+\infty\right) \text { e } x, y \in \mathscr{B}_{a},
$$

onde $\mathscr{B}_{a}=\left\{z \in \mathbb{R}^{n}:\|z\|<a\right\}$;

(iii) Existe uma função monótona crescente $b: \mathbb{R}_{+} \rightarrow \mathbb{R}_{+}$tal que $b(0)=0 e$

$$
U(t, y(t)) \geq b\left(\left\|y_{t}\right\|\right)
$$

para qualquer $y \in G^{-}\left(\left[t_{0}-r,+\infty\right), \mathbb{R}^{n}\right), t \in\left[t_{0},+\infty\right)$;

(iv) Existe uma função $\Lambda: \mathbb{R}_{+} \rightarrow \mathbb{R}_{+}$tal que

$$
D^{+} U(t, \psi(0)) \leq-\Lambda(|\psi(0)|), \quad \text { sempre que } U(t+\theta, \psi(\theta)) \leq U(t, \psi(0))
$$

para $t \in\left[t_{0},+\infty\right), \theta \in[-r, 0] e \psi \in G^{-}\left([-r, 0], \mathbb{R}^{n}\right)$.

Então a solução trivial y $\equiv 0$ de (4.1) será uniformemente estável.

Demonstração. Para $s \geq t_{0}$ e $\xi \in \bar{E}_{\rho}$, definamos

$$
\bar{U}(s, \xi)=\sup _{\theta \in[-r, 0]} U(s+\theta, \xi(\theta)) .
$$

É claro que $\bar{U}(s, 0)=0$ para todo $s \geq t_{0}$.

Dados $t \geq t_{0}$ e $\psi \in \bar{E}_{\rho}$, consideremos $y:[t-r,+\infty) \rightarrow \mathbb{R}^{n}$ uma solução de (4.1) tal que $y_{t}=\psi$. Note que, pelo Lema (1.16), a solução $x$ da EDO generalizada (3.20) correspondente a EDFR impulsiva (4.1) é contínua à esquerda e, portanto, $y$ também o é. Este fato pode ser facilmente constatado pelas relações entre as soluções $y$ e $x$ dadas nos Teoremas 3.5 e 3.6. Sendo assim, as hipóteses do teorema presente implicam que a função $U(s, y(s))$ é contínua à esquerda para $s$ no intervalo de definição de $y$.

Por (4.22), temos

$$
\bar{U}(t, \psi)=\bar{U}\left(t, y_{t}\right)=\sup _{\theta \in[-r, 0]} U(t+\theta, y(t+\theta)) .
$$

Mostraremos que $D^{+} \bar{U}(t, \psi)=D^{+} \bar{U}\left(t, y_{t}\right) \leq 0$.

Seja

$$
R(t)=\{U(t+\theta, y(t+\theta)): \theta \in[-r, 0]\} .
$$


Consideraremos dois casos: quando $\bar{U}\left(t, y_{t}\right)$ pertence a $R(t)$ e caso contrário.

Suponhamos que $\bar{U}\left(t, y_{t}\right)$ pertença a $R(t)$. Então existe $\theta_{0} \in[-r, 0]$ tal que $U\left(t+\theta_{0}, y\left(t+\theta_{0}\right)\right)=$ $\bar{U}\left(t, y_{t}\right)$. Se $\theta_{0}=0$, então $\bar{U}\left(t, y_{t}\right)=U(t, y(t))$, donde segue, pela condição $(i v)$, que $D^{+} \bar{U}\left(t, y_{t}\right) \leq 0$. Se $\theta_{0}<0$, então $U(t+\theta, y(t+\theta))<U\left(t+\theta_{0}, y\left(t+\theta_{0}\right)\right)$ para todo $\theta_{0}<\theta \leq 0$, por (4.23) e pela escolha de $\theta_{0}$. Então para $h>0$ suficientemente pequeno com $h<\left|\theta_{0}\right|$, temos

$$
\begin{aligned}
\bar{U}\left(t+h, y_{t+h}\right) & =\sup _{\theta \in[-r, 0]} U(t+h+\theta, y(t+h+\theta)) \\
& =U\left(t+\theta_{0}, y\left(t+\theta_{0}\right)\right)=\bar{U}\left(t, y_{t}\right)
\end{aligned}
$$

e portanto $D^{+} \bar{U}\left(t, y_{t}\right)=0$.

Agora, consideremos o caso em que $\bar{U}\left(t, y_{t}\right)$ não pertence a $R(t)$. Neste caso,

$$
\bar{U}\left(t, y_{t}\right)>U(t+\theta, y(t+\theta)), \quad \theta \in[-r, 0],
$$

e existe uma sequência convergente $\left\{\theta_{n}\right\}_{n \in \mathbb{N}}$ em $[-r, 0]$, com $\bar{\theta}=\lim _{n \rightarrow+\infty} \theta_{n}$, tal que

$$
\bar{U}\left(t, y_{t}\right)=\lim _{n \rightarrow+\infty} U\left(t+\theta_{n}, y\left(t+\theta_{n}\right)\right) .
$$

Suponhamos que existam infinitos $\theta_{n_{k}}$ 's de forma que $\theta_{n_{k}}<\bar{\theta}$ para todo $k$. Então

$$
\bar{U}\left(t, y_{t}\right)=\lim _{k \rightarrow+\infty} U\left(t+\theta_{n_{k}}, y\left(t+\theta_{n_{k}}\right)\right)=U(t+\bar{\theta}, y(t+\bar{\theta}))
$$

o que é uma contradição, já que $\bar{\theta} \in[-r, 0] \mathrm{e}$, em tal caso, $\bar{U}\left(t, y_{t}\right)$ pertenceria a $R(t)$. Este fato também mostra que $\bar{\theta}<0$. Portanto, suporemos que $\bar{\theta}<\theta_{n}<0$, para todo $n$, e então

$$
\bar{U}\left(t, y_{t}\right)=U((t+\bar{\theta})+, y((t+\bar{\theta})+))
$$

Logo, para $h>0$ suficientemente pequeno com $h<|\bar{\theta}|$, temos

$$
\begin{aligned}
\bar{U}\left(t+h, y_{t+h}\right) & =\sup _{\theta \in[-r, 0]} U(t+h+\theta, y(t+h+\theta)) \\
& =U((t+\bar{\theta})+, y((t+\bar{\theta})+))=\bar{U}\left(t, y_{t}\right) .
\end{aligned}
$$

Então $D^{+} \bar{U}\left(t, y_{t}\right)=0$. 
Agora, afirmamos que $\bar{U}$ satisfaz a condição $(i i)$ do Teorema 4.7. Com efeito, consideremos $t \geq t_{0}$. Dadas funções $\widehat{\psi}, \bar{\psi} \in \overline{E_{\rho}}$, sejam $\widehat{y}, \bar{y}$ as soluções de (4.1) tais que $\widehat{y}_{t}=\widehat{\psi}, \bar{y}_{t}=\bar{\psi}$. Com a notação acima, temos

$$
\begin{gathered}
\bar{U}(t, \widehat{\psi})=\bar{U}\left(t, \widehat{y}_{t}\right)=U\left(t+\theta_{\widehat{\psi}}, \widehat{y}\left(t+\theta_{\widehat{\psi}}\right)\right) \quad \text { ou } \\
\bar{U}(t, \widehat{\psi})=\bar{U}\left(t, \widehat{y}_{t}\right)=U\left(\left(t+\bar{\theta}_{\widehat{\psi}}\right)+, \widehat{y}\left(\left(t+\bar{\theta}_{\widehat{\psi}}\right)+\right)\right)
\end{gathered}
$$

e

$$
\begin{gathered}
\bar{U}(t, \bar{\psi})=\bar{U}\left(t, \bar{y}_{t}\right)=U\left(t+\theta_{\bar{\psi}}, \bar{y}\left(t+\theta_{\bar{\psi}}\right)\right) \quad \text { ou } \\
\bar{U}(t, \bar{\psi})=\bar{U}\left(t, \bar{y}_{t}\right)=U\left(\left(t+\bar{\theta}_{\bar{\psi}}\right)+, \bar{y}\left(\left(t+\bar{\theta}_{\bar{\psi}}\right)+\right)\right),
\end{gathered}
$$

onde $\theta_{\widehat{\psi}}, \theta_{\bar{\psi}}$ e $\bar{\theta}_{\widehat{\psi}}, \bar{\theta}_{\bar{\psi}}$ correspondem respectivamente a $\theta_{0}$ e $\bar{\theta}$ para as funções $\widehat{\psi}, \bar{\psi} \in \overline{E_{\rho}}$.

Consideremos $\overline{\mathscr{B}}_{\rho}=\left\{x \in \mathbb{R}^{n}:\|x\| \leq \rho\right\}$ e $\mathscr{B}_{c}=\left\{x \in \mathbb{R}^{n}:\|x\|<c\right\}$, onde $\rho<c$. Como

$$
U\left(\left(t+\bar{\theta}_{\widehat{\psi}}\right)+, \widehat{y}\left(\left(t+\bar{\theta}_{\widehat{\psi}}\right)+\right)\right) \text { e } U\left(\left(t+\bar{\theta}_{\bar{\psi}}\right)+, \bar{y}\left(\left(t+\bar{\theta}_{\bar{\psi}}\right)+\right)\right)
$$

existem, a condição (ii) implica

$$
\begin{aligned}
|\bar{U}(t, \widehat{\psi})-\bar{U}(t, \bar{\psi})| & =\left|\bar{U}\left(t, \widehat{y}_{t}\right)-\bar{U}\left(t, \bar{y}_{t}\right)\right| \\
& =\left|\sup _{\theta \in[-r, 0]} U(t+\theta, \widehat{y}(t+\theta))-\sup _{\theta \in[-r, 0]} U(t+\theta, \bar{y}(t+\theta))\right| \\
& \leq \sup _{\theta \in[-r, 0]}|U(t+\theta, \widehat{y}(t+\theta))-U(t+\theta, \bar{y}(t+\theta))| \\
& \leq K_{c} \sup _{\theta \in[-r, 0]}\|\widehat{y}(t+\theta)-\bar{y}(t+\theta)\| \\
& =K_{c}\|\widehat{y}-\bar{y}\|=K_{c}\|\widehat{\psi}-\bar{\psi}\|,
\end{aligned}
$$

já que $\widehat{y}(t+\theta), \bar{y}(t+\theta) \in \overline{\mathscr{B}}_{\rho}$, para todo $\theta \in[-r, 0]$, e $\overline{\mathscr{B}}_{\rho} \subset \mathscr{B}_{c}$.

Além disso, temos

$$
\bar{U}(t, \psi)=\bar{U}\left(t, y_{t}\right) \geq U(t, y(t)) \geq b\left(\left\|y_{t}\right\|\right)=b(\|\psi\|),
$$

pela definição de $\bar{U}$ e pela condição $(i i i)$.

Desta forma, $\bar{U}$ é um funcional de Lyapunov e todas as condições do Teorema 4.7 estão satisfeitas para $\bar{U}$ e, portanto, a solução trivial de (4.1) é uniformemente estável. 
Teorema 4.10 ([2], Teorema 4.9). Considere a EDFR impulsiva (4.1). Seja $U:\left[t_{0}-r,+\infty\right) \times \mathbb{R}^{n} \rightarrow \mathbb{R}$ uma função contínua à esquerda em $\left(t_{0}-r,+\infty\right)$. Suponha que os limites

$$
U(t-, y(t-))=\lim _{s \rightarrow t^{-}} U(s, y(s)), \quad t \in\left(t_{0}-r,+\infty\right)
$$

$e$

$$
U(t+, y(t+))=\lim _{s \rightarrow t^{+}} U(s, y(s)), \quad t \in\left[t_{0}-r,+\infty\right),
$$

existam, com $U(t-, y(t-))=U(t, y(t))$, para $y \in G^{-}\left(\left[t_{0}-r,+\infty\right), \mathbb{R}^{n}\right)$. Suponha que as condições $(i),(i i)$ $e$ (iii) do Teorema 4.9 estejam satisfeitas e que exista uma função monótona crescente $d: \mathbb{R}_{+} \rightarrow \mathbb{R}_{+}$tal que $d(0)=0$ e para toda solução y de (4.1), tenhamos

$$
\sup _{\theta \in[-r, 0]} U(s+\theta, y(s+\theta)) \leq d(|y(s)|)
$$

onde $s \geq t_{0}$, com $d(\bar{t}) \geq b(\bar{t})$, para todo $\bar{t} \geq 0$. Suponha, ainda, que exista uma função $\Lambda: \mathbb{R}_{+} \rightarrow \mathbb{R}_{+}$que satisfaça $\Lambda(0)=0$ e $\Lambda(x)>0$, se $x \neq 0$, e que exista uma função contínua e não decrescente $p(s)>s$ para $s>0$ tal que

$$
D^{+} U(t, \psi(0)) \leq-\Lambda(|\psi(0)|) \quad \text { se } U(t+\theta, \psi(\theta))<p(U(t, \psi(0))
$$

para $\theta \in[-r, 0], t \in\left[t_{0},+\infty\right) e \psi \in G^{-}\left([-r, 0], \mathbb{R}^{n}\right)$. Então a solução trivial $y \equiv 0$ de (4.1) será uniformemente assintoticamente estável.

Demonstração. Esta prova segue as ideias da prova apresentada por J. Hale e S. M. Verduyn Lunel para o Teorema 5.4.2 em [15].

Se definirmos

$$
\bar{U}(s, \xi)=\sup _{\theta \in[-r, 0]} U(s+\theta, \xi(\theta)),
$$

para $s \geq t_{0}$ e $\xi \in \bar{E}_{\rho}$, concluiremos que a solução trivial $y \equiv 0$ é uniformemente estável, por uma repetição de argumentos usados na prova do Teorema 4.9.

Sejam $t_{0} \geq 0$ e $\phi \in E_{\rho}$. Seja $\bar{y}:\left[t_{0}-r,+\infty\right) \rightarrow \mathbb{R}^{n}$ a solução de (4.1) que satisfaz $\bar{y}_{t_{0}}=\phi$. Escrevemos $\bar{y}(t)=\bar{y}\left(t, t_{0}, \phi\right)$ para denotar esta solução.

Consideremos $\bar{y}_{t}=\bar{y}_{t}\left(t_{0}, \phi\right)$ para $t \geq t_{0}$, e tomemos $\varepsilon>0$. Como a solução $y \equiv 0$ de (4.1) é uniformemente estável, existe $\delta>0$ tal que se $\|\phi\|<\delta$, então $\left\|\bar{y}_{t}\left(t_{0}, \phi\right)\right\|<\varepsilon$. Note que $\|\phi\|<\delta$ implica 
$U(t, \bar{y}(t))<d(\varepsilon)$, para todo $t \in\left[t_{0},+\infty\right)$, já que

$$
U(t, \bar{y}(t)) \leq \sup _{\theta \in[-r, 0]} U(t+\theta, \bar{y}(t+\theta)) \leq d(|\bar{y}(t)|) \leq d\left(\left\|\bar{y}_{t}\right\|\right)<d(\varepsilon)
$$

e $d$ é uma função crescente.

Suponhamos que $0<\eta \leq \varepsilon$ seja arbitrário. Vamos mostrar que existe um número $T=T(\varepsilon, \eta)>0$ tal que $\|\phi\|<\delta$ implica $\left\|\bar{y}_{t}\right\| \leq \eta$, para todo $t \in\left[t_{0}+T,+\infty\right)$. Para isso, é suficiente mostrar que $U(t, \bar{y}(t)) \leq$ $b(\eta)$, para todo $t \in\left[t_{0}+T,+\infty\right)$, onde $b$ é dada pela condição (iii) do Teorema 4.9.

Primeiramente, vamos encontrar o número $T$. Pelas propriedades da função $p(s)$, existe um número $\alpha>0$ tal que $p(s)-s \geq \alpha$ para $b(\eta) \leq s \leq d(\varepsilon)$ (note que $b(\eta) \leq b(\varepsilon) \leq d(\varepsilon)$ ).

Seja $z$ o primeiro inteiro positivo tal que $b(\eta)+z \alpha>d(\varepsilon)$. Como $b(\eta) \leq d(\varepsilon)$, temos $d^{-1}(b(\eta)) \leq$ $\varepsilon$. Seja

$$
\beta=\inf _{d^{-1}(b(\eta)) \leq s \leq \varepsilon} \Lambda(s)>0
$$

e defina

$$
T:=\frac{z d(\varepsilon)}{\beta}
$$

Agora, vamos mostrar que $U(t, \bar{y}(t)) \leq b(\eta)$, para todo $t \in\left[t_{0}+\frac{d(\varepsilon)}{\beta},+\infty\right)$. Afirmamos que $U(t, \bar{y}(t)) \leq$ $b(\eta)+(z-1) \alpha$, para todo $t \in\left[t_{0}+\frac{d(\varepsilon)}{\beta},+\infty\right)$. De fato, suponhamos que $b(\eta)+(z-1) \alpha<U(t, \bar{y}(t))$, para $t \in\left[t_{0}, t_{0}+\frac{d(\varepsilon)}{\beta}\right]$. Pela escolha de $z$ e por (4.27), temos

$$
b(\eta) \leq b(\eta)+(z-1) \alpha<U(t, \bar{y}(t))<d(\varepsilon)
$$

e

$$
p(U(t, \bar{y}(t)) \geq U(t, \bar{y}(t))+\alpha>b(\eta)+z \alpha>d(\varepsilon)>U(t+\theta, \bar{y}(t+\theta)),
$$

para $t_{0} \leq t \leq t_{0}+\frac{d(\varepsilon)}{\beta}$ e $\theta \in[-r, 0]$. Note que (4.27) e (4.28) implicam

$$
d^{-1}(b(\eta))<d^{-1}(d(|\bar{y}(t)|))<d^{-1}(d(\varepsilon)),
$$

isto é,

$$
d^{-1}(b(\eta))<|\bar{y}(t)|<\varepsilon,
$$


onde $t_{0} \leq t \leq t_{0}+\frac{d(\varepsilon)}{\beta}$. Consequentemente, por (4.25), obtemos

$$
D^{+} U(t, \bar{y}(t)) \leq-\Lambda(|\bar{y}(t)|) \leq-\beta, \quad t_{0} \leq t \leq t_{0}+\frac{d(\varepsilon)}{\beta}
$$

Logo,

$$
U\left(t_{1}, \bar{y}\left(t_{1}\right)\right) \leq U\left(t_{0}, \bar{y}\left(t_{0}\right)\right)-\beta\left(t_{1}-t_{0}\right)<d(\varepsilon)-\beta\left(t_{1}-t_{0}\right)
$$

e $U\left(t_{1}, \bar{y}\left(t_{1}\right)\right)<0$, onde $t_{1}=t_{0}+\frac{d(\varepsilon)}{\beta}$, o que é uma contradição, visto que $U$ é definida positiva, pela condição (iii). Então,

$$
U(t, \bar{y}(t)) \leq b(\eta)+(z-1) \alpha, \quad t=t_{0}+\frac{d(\varepsilon)}{\beta}
$$

Note que, quando $U(t, \bar{y}(t))=b(\eta)+(z-1) \alpha$, temos $D^{+} U(t, \bar{y}(t)) \leq 0$, por (4.25), uma vez que $b(\eta) \leq U(t, \bar{y}(t))=b(\eta)+(z-1) \alpha<d(\varepsilon)$ e, portanto,

$$
p(U(t, \bar{y}(t)) \geq U(t, \bar{y}(t))+\alpha=b(\eta)+z \alpha>d(\varepsilon)>U(t+\theta, \bar{y}(t+\theta)),
$$

para $\theta \in[-r, 0]$.

Agora, suponhamos que exista $\bar{t}>t_{0}+\frac{d(\varepsilon)}{\beta}$ tal que $U(\bar{t}, \bar{y}(\bar{t}))>b(\eta)+(z-1) \alpha$. Então $D^{+} U(t, \bar{y}(t))>$ 0 , para $t$ tal que $U(t, \bar{y}(t))=b(\eta)+(z-1) \alpha$, o que é uma contradição. É importante observar que se $\bar{t}=t_{k}^{i}$, ou seja, se $\bar{t}$ for um momento de impulso, obtemos a mesma contradição.

Sejam $\bar{t}_{n}=\frac{n d(\varepsilon)}{\beta}, n=1, \cdots, z, \bar{t}_{0}=0$ e suponhamos que para algum inteiro $N \geq 1$ e para $t$ satisfazendo $\bar{t}_{n-1} \leq t-t_{0} \leq \bar{t}_{n}$, tenhamos

$$
b(\eta)+(z-N) \alpha<U(t, \bar{y}(t)) \leq b(\eta)+(z-N+1) \alpha .
$$

Usando argumentos anteriores, obtemos

$$
D^{+} U(t, \bar{y}(t)) \leq-\beta, \quad \bar{t}_{n-1} \leq t-t_{0} \leq \bar{t}_{n}
$$

e

$$
U(t, \bar{y}(t)) \leq U\left(t_{0}+\bar{t}_{n-1}, \bar{y}\left(t_{0}+\bar{t}_{n-1}\right)\right)-\beta\left(t-t_{0}-\bar{t}_{n-1}\right)<d(\varepsilon)-\beta\left(t-t_{0}-\bar{t}_{n-1}\right) .
$$

Logo $U(t, \bar{y}(t))<0$, sempre que $t=t_{0}+\bar{t}_{n}$. Analogamente, podemos provar que $U(t, \bar{y}(t)) \leq b(\eta)+$ $(z-N) \alpha$, para $t \geq t_{0}+\bar{t}_{n}$. Para $N=z$, temos $U(t, \bar{y}(t)) \leq b(\eta)$ para todo $t \geq t_{0}+\frac{z d(\varepsilon)}{\beta}$. 
Finalmente, como

$$
b\left(\left\|\bar{y}_{t}\right\|\right) \leq U(t, \bar{y}(t)) \leq b(\eta)
$$

e $b$ é uma função crescente, temos $\left\|\bar{y}_{t}\right\| \leq \eta$, para todo $t \geq t_{0}+\frac{z d(\varepsilon)}{\beta}$, o que finaliza a prova.

\subsubsection{Comentários e observações}

Muitos problemas em física, mecânica, eletrônica, biologia, economia, medicina, farmacocinética e várias outras ciências podem ser modelados como casos especiais do sistema

$$
\left\{\begin{array}{l}
\dot{y}(t)=f\left(y_{t}, y, t\right), t \neq \tau_{k}(y(t)), \quad t \geq t_{0} \\
\Delta y\left(t_{k}\right)=I_{k}\left(y\left(t_{k}\right)\right), t=\tau_{k}(y(t)), \quad k=0,1,2, \ldots \\
f(0,0, t) \equiv 0, I_{k}(0)=0, k=0,1,2, \ldots \\
y_{t_{0}}=\phi
\end{array}\right.
$$

e ainda valem resultados de estabilidade similares aos Teoremas 4.9 e 4.10. Tais resultados podem ser facilmente obtidos. De fato, no caso do sistema (4.29), é suficiente substituir (3.3) por

$$
F(y, t)(\vartheta)=\left\{\begin{array}{l}
0, t_{0}-r \leq \vartheta \leq t_{0}, \\
\int_{t_{0}}^{\vartheta} f\left(y_{s}, y(s), s\right) d s, t_{0} \leq \vartheta \leq t<+\infty \\
\int_{t_{0}}^{t} f\left(y_{s}, y(s), s\right) d s, \quad t_{0} \leq t \leq \vartheta<+\infty .
\end{array}\right.
$$

Considera-se $J$ dada por (3.10) e, então, as equações (4.29) e (3.20) devem compor $G$ dada por (3.17). A prova deste fato segue como em [8], Teoremas 3.4 e 3.5 aplicando as observações da última seção de [8].

Em particular, no caso não impulsivo (isto é, quando $I_{k}(x) \equiv 0$, para todo $k$ ), existe uma série de problemas modelados através de formas específicas de $\dot{y}(t)=f\left(y_{t}, y, t\right)$. Em dinâmica de população, por exemplo, podemos citar o modelo de Lasota-Wazewska

$$
N^{\prime}(t)=-\mu N(t)+p e^{-\sigma N(t-r)}
$$

a equação de varejeiras de Nicholson

$$
N^{\prime}(t)=-\delta N(t)+p N(t-r) e^{-a N(t-r)}
$$


e a equação de Nazarenko

$$
x^{\prime}(t)=-p x(t)+\frac{q x(t)}{\sigma+x^{n}(t-r)} .
$$

Veja, por exemplo, [5], [13], [14], [20], [24] para referências com respeito a estes modelos, os quais também podem ser considerados como estando sujeitos a momentos variáveis de efeitos de impulso (ou controles). Por isso, é importante ter os resultados de estabilidade para tais equações.

Pode-se considerar, ainda, que a equação (4.29) seja dada como equação diferencial diferença com retardo variável, com lado direito dado por $f(y(t-r(t)), y, t)$, onde $r(t)$ é uma função de $t$ tal que $r^{\prime}(t)<$ $\Delta<1$ para algum $\Delta>0$. Neste caso, consideraremos $G^{-}\left([-r(t), 0], \mathbb{R}^{n}\right)$ ao invés de $G^{-}\left([-r, 0], \mathbb{R}^{n}\right)$ e todas as outras alterações adequadas. Também $F(y, t)$ será dada por

$$
F(y, t)(\vartheta)=\left\{\begin{array}{l}
0, t_{0}-r(t) \leq \vartheta \leq t_{0}, \\
\int_{t_{0}}^{\vartheta} f(y(s-r(s)), y(s), s) d s, \quad t_{0} \leq \vartheta \leq t<+\infty, \\
\int_{t_{0}}^{t} f(y(s-r(s)), y(s), s) d s, t_{0} \leq t \leq \vartheta<+\infty .
\end{array}\right.
$$

Em seguida, as extensões para o caso onde há ocorrência de vários retardos podem ser obtidas de forma semelhante.

Consideremos a equação com retardamento

$$
\dot{y}_{i}=-c_{i} y_{i}(t)+\sum_{j=1}^{n} a_{i j} g_{j}\left(y_{j}(t)\right)+\sum_{j=1}^{n} b_{i j} f\left(y_{j}\left(t-r_{i j}(t)\right)\right)+H_{i}(t), \quad i=1,2, \ldots, n
$$

onde $c_{i}>0,0 \leq r_{i j}(t) \leq r$. A equação (4.30) descreve o processo de evolução e redes neurais, onde $n$ corresponde ao número de unidades das redes neurais, $y_{i}$ corresponde a variável de estado, $f_{j}\left(x_{j}\right), g_{j}\left(x_{j}\right)$ são funções de ativação dos neurônios, $c_{i}$ é a constante de mudança do neurônio, $a_{i j}, b_{i j}$ são os pesos das interconexões de neurônios, $H_{i}$ é a polarização interna, e $r_{i j}(t)$ é o retardo de transmissão de informação. Em particular, a equação (4.30) generaliza modelos como a rede neural de Hopfield, redes bidirecionais neurais, redes neurais celulares, redes neurais recorrentes, etc. Veja [6], [18], [23], [30] e [31] como referência para tais modelos.

Fazendo as tranformações necessárias, a equação (4.30) pode ser formulada como

$$
\dot{y}(t)=f\left(y\left(t-r_{1}(t)\right), y\left(t-r_{2}(t)\right), y\left(t-r_{3}(t)\right), y(t), t\right),
$$

mas com vários retardos $r_{i j}(t)$. Logo resultados semelhantes aos Teoremas 4.9 e 4.10 podem ser obtidos para esse tipo de equação, considerando-se mudanças bruscas de estado em momentos fixos ou em 
momentos variáveis de tempo como em (4.29).

\subsection{Estabilidade exponencial da solução trivial}

Como na seção anterior, suporemos que

$$
f(0, t)=0 \text { para todo } t \text { e } I_{k}(0)=0, k=0,1,2, \ldots,
$$

para que a função $y \equiv 0$ possa ser considerada uma solução da EDFR impulsiva (4.1) em qualquer intervalo contido em $\left[t_{0},+\infty\right)$. Também consideraremos o conjunto $E_{c}=\left\{\psi \in G^{-}\left([-r, 0], \mathbb{R}^{n}\right):\|\psi\|<\right.$ $c\}, c>0$.

A seguir, apresentaremos o conceito de estabilidade exponencial para a equação (4.1), o qual está presente em [11].

Definição 4.11. Seja $\lambda$ uma constante positiva. A solução trivial de (4.1) será dita exponencialmente estável se, para quaiquer $t_{0} \geq 0, \varepsilon>0$, existir $\delta=\delta(\varepsilon)>0$ tal que se $\phi \in E_{c} e \bar{y}:\left[t_{0}-r,+\infty\right) \rightarrow \mathbb{R}^{n}$ for solução de (4.1) tal que $\bar{y}_{t_{0}}=\phi e$

$$
\|\phi\|<\delta
$$

então

$$
\left|\bar{y}\left(t, t_{0}, \phi\right)\right|<\varepsilon \exp \left[-\lambda\left(t-t_{0}\right)\right], \quad \text { para todo } t \geq t_{0} .
$$

Usando a relação (4.3), a corresponência entre as equações (4.1) e (3.20) e o Teorema 2.9, obtemos o seguinte resultado sobre estabilidade exponencial para EDFRs com impulso em tempo variável.

Teorema 4.12 ([2], Teorema 4.6). Considere a EDFR impulsiva (4.1). Suponha que a função $U$ : $\left[t_{0},+\infty\right) \times \bar{E}_{\rho} \rightarrow \mathbb{R}$ seja um funcional de Lyapunov e que as seguintes condições sejam válidas:

(i) $U(t, 0)=0, t \in\left[t_{0},+\infty\right)$;

(ii) Existe uma constante $K>0$ tal que

$$
|U(t, \psi)-U(t, \bar{\psi})| \leq K\|\psi-\bar{\psi}\|, \quad t \in\left[t_{0},+\infty\right), \psi, \bar{\psi} \in \bar{E}_{\rho}
$$

Então a solução trivial y $\equiv 0$ de (4.1) será exponencialmente estável. 
Demonstração. Visto que $f$ satisfaz as condições $(A)$ e $(B)$ e $I_{k}$ satisfaz $\left(A^{\prime}\right)$ e $\left(B^{\prime}\right)$ para $k=0,1,2, \ldots$, a função $G$ na equação (3.20) pertence à classe $\mathscr{F}(\Omega, h)$, onde $\Omega=P C_{1} \times\left[t_{0},+\infty\right)$ e $h=h_{1}+h_{2}$, com $h_{1}$ dada por (3.7) e $h_{2}$ dada por (3.14).

Consideremos $V:\left[t_{0},+\infty\right) \times \bar{B}_{\rho} \rightarrow \mathbb{R}$ dada por (4.3). Pelo Lema 4.5,

$$
V(t, 0)=0, \quad \text { para } t \in\left[t_{0},+\infty\right)
$$

$\mathrm{e}$

$$
|V(t, z)-V(t, \bar{z})| \leq K\|z-\bar{z}\|, \quad \text { para } t \in\left[t_{0},+\infty\right) \text { e } z, \bar{z} \in \bar{B}_{\rho} .
$$

Além disso, pela Observação 4.3, $V$ é um funcional de Lyapunov. Portanto, as hipóteses do Teorema 2.9 estão satisfeitas. Logo, fixado $\lambda>0$, para todo $\varepsilon>0$, existe $\delta=\delta(\varepsilon)>0$ tal que se $\bar{x}:[\gamma, v] \rightarrow B_{\rho}$, $t_{0} \leq \gamma<v<+\infty$, for uma função de variação limitada em $[\gamma, v]$ e contínua à esquerda em $(\gamma, v]$ tal que

$$
\|\bar{x}(\gamma)\|<\delta
$$

$\mathrm{e}$

$$
\operatorname{var}_{\gamma}^{v}\left(\bar{x}(s)-\int_{\gamma}^{s} D G(\bar{x}(\tau), t)\right)<\delta
$$

então

$$
\|\bar{x}(t)\|<\varepsilon \exp [-\lambda(t-\gamma)], \quad t \in[\gamma, v] .
$$

Seja $\phi \in \bar{E}_{\rho}$ e $\bar{y}:\left[t_{0}-r,+\infty\right) \rightarrow \mathbb{R}^{n}$ uma solução de (4.1) tal que $\bar{y}_{t_{0}}=\phi$ e

$$
\|\phi\|<\delta
$$

Queremos provar que

$$
\left|\bar{y}\left(t, t_{0}, \phi\right)\right|<\varepsilon \exp \left[-\lambda\left(t-t_{0}\right)\right], \quad t \in\left[t_{0},+\infty\right)
$$

Vamos denotar $\bar{y}_{t}=\bar{y}_{t}\left(t_{0}, \phi\right)$ e definir

$$
\bar{x}(t)(\tau)=\left\{\begin{array}{l}
\bar{y}(\tau), t_{0}-r \leq \tau \leq t \\
\bar{y}(t), \tau \geq t
\end{array}\right.
$$

Pelo Teorema 3.5, $\bar{x}(t)$ é uma solução em $\left[t_{0},+\infty\right)$ da EDO generalizada (3.20) satisfazendo a condição 
inicial $\bar{x}\left(t_{0}\right)=\widetilde{x}$, onde

$$
\widetilde{x}(\tau)= \begin{cases}\phi\left(\tau-t_{0}\right), & t_{0}-r \leq \tau \leq t_{0}, \\ \phi(0), & \tau \geq t_{0} .\end{cases}
$$

Além disso, $\bar{x}$ é contínua à esquerda em $\left(t_{0},+\infty\right)$ e de variação limitada em $\left[t_{0},+\infty\right)$.

Por (4.35) e (4.32), temos

$$
\left\|\bar{x}\left(t_{0}\right)\right\|=\sup _{t_{0}-r \leq \tau<+\infty}|\widetilde{x}(\tau)|=\|\phi\|<\delta .
$$

E mais, para qualquer $v \in\left[t_{0},+\infty\right)$, temos

$$
\operatorname{var}_{t_{0}}^{v}\left(\bar{x}(s)-\int_{t_{0}}^{s} D G(\bar{x}(\tau), t)\right)=0<\delta
$$

Portanto vale (4.31), ou seja, $\|\bar{x}(t)\|<\varepsilon \exp \left[-\lambda\left(t-t_{0}\right)\right]$ para todo $t \in\left[t_{0}, v\right]$. Em particular,

$$
\|\bar{x}(v)\|<\varepsilon \exp \left[-\lambda\left(v-t_{0}\right)\right]
$$

Então (4.34) implica que, para qualquer $t \in\left[t_{0}, v\right]$, temos

$$
\begin{aligned}
|\bar{y}(t)| & \leq\left\|\bar{y}_{t}\right\|=\sup _{-r \leq \theta \leq 0}|\bar{y}(t+\theta)| \leq \sup _{t_{0}-r \leq \tau \leq v}|\bar{y}(\tau)| \\
& =\sup _{t_{0}-r \leq \tau \leq v}|\bar{x}(v)(\tau)|=\sup _{t_{0}-r \leq \tau<+\infty}|\bar{x}(v)(\tau)| \\
& =\|\bar{x}(v)\|<\varepsilon \exp \left[-\lambda\left(v-t_{0}\right)\right]<\varepsilon \exp \left[-\lambda\left(t-t_{0}\right)\right] .
\end{aligned}
$$

Como vé arbitrário, vale (4.33) e a prova está completa.

\subsection{Limitação uniforme de soluções}

Lembrando que o Teorema 3.7 nos garante existência local e unicidade de solução de (4.1)-(4.2), consideraremos $y:\left[t_{0}-r,+\infty\right) \rightarrow \mathbb{R}^{n}$ a solução de tal problema de valor inicial que será denotada por $y(t)=y\left(t, t_{0}, \phi\right)$.

Definição 4.13. A solução $y(t)=y\left(t, t_{0}, \phi\right)$ do sistema (4.1)-(4.2) será dita 
(i) Uniformemente limitada, se para todo $\alpha>0$, existir $M=M(\alpha)>0$ tal que se

$$
\|\phi\|<\alpha
$$

então

$$
|y(t)|<M, \quad t \geq t_{0}
$$

(ii) Quase uniformemente eventualmente limitada, se existir uma constante B $>0$ tal que para todo $\alpha>0$, existe $T=T(\alpha)>0$ tal que se

$$
\|\phi\|<\alpha
$$

então

$$
|y(t)|<B, \quad t \geq t_{0}+T .
$$

(iii) Uniformemente eventualmente limitada, se for uniformemente limitada e quase uniformemente eventualmente limitada.

Nós aplicaremos os Teoremas 3.5 e 3.6 juntamente com os Teoremas 2.11 e 2.12 a fim de obtermos resultados de limitação uniforme para a solução do problema (4.1)-(4.2).

Prosseguiremos como na prova do Lema 4.5 para o provar o resultado auxiliar abaixo.

Lema 4.14 ([1], Lema 5.3). Considere a EDFR impulsiva (4.1) e suponha que a função $U:\left[t_{0},+\infty\right) \times$ $G^{-}\left([-r, 0], \mathbb{R}^{n}\right) \rightarrow \mathbb{R}$ satisfaça as seguintes condições:

(i) $U(t, 0)=0$, para todo $t \in\left[t_{0},+\infty\right)$;

(ii) Para cada $a>0$, existe uma constante $K_{a}>0$ tal que

$$
|U(t, \psi)-U(t, \bar{\psi})| \leq K_{a}\|\psi-\bar{\psi}\|, \quad t \in\left[t_{0},+\infty\right), \psi, \bar{\psi} \in \mathscr{B}_{a}
$$

onde $\mathscr{B}_{a}=\left\{\phi \in G^{-}\left([-r, 0], \mathbb{R}^{n}\right):\|\phi\|<a\right\}$. Então a função $V$ definida por (4.3) satisfaz $V(t, 0)=0$ para todo $t \in\left[t_{0},+\infty\right), e$

$$
|V(t, x)-V(t, \bar{x})| \leq K_{a}\|x-\bar{x}\|,
$$

para $t \geq t_{0}$ e $x, \bar{x} \in B_{a}$, onde $B_{a}=\left\{\psi \in P C_{1}:\|\psi\|<a\right\}$.

Demonstração. Dado $t \geq t_{0}$, sejam $y, \bar{y}, \widehat{y}:[t-r,+\infty) \rightarrow \mathbb{R}^{n}$ soluções da equação (4.1) com condições iniciais $y_{t}=\psi, \bar{y}_{t}=\bar{\psi}$ e $\widehat{y}_{t}=0$. Suponhamos que $x, \bar{x}, \widehat{x}$ sejam as soluções em $[t,+\infty)$ da EDO generalizada 
(3.20) dadas pelo Teorema 3.5 que são correspondentes a $y, \bar{y}$ e $\widehat{y}$ respectivamente. Então $(x(t))_{t}=y_{t}=\psi$, $(\bar{x}(t))_{t}=\bar{y}_{t}=\bar{\psi} \mathrm{e}(\widehat{x}(t))_{t}=\widehat{y}_{t}=0$.

Note que, como $f$ satisfaz as condições $(A)$ e $(B)$ e $I_{k}$ satisfaz as condições $\left(A^{\prime}\right)$ e $\left(B^{\prime}\right)$ para $k=$ $0,1,2, \ldots$, a função $G$ na equação (3.20) pertence à classe $\mathscr{F}(\Omega, h)$, onde $\Omega=P C_{1} \times\left[t_{0},+\infty\right)$ e $h=$ $h_{1}+h_{2}$, com $h_{1}$ dada por (3.7) e $h_{2}$ dada por (3.14).

Seja $V:\left[t_{0},+\infty\right) \times P C_{1} \rightarrow \mathbb{R}$ dada por (4.3). Pela condição $(i)$, temos

$$
0=U(t, 0)=U\left(t, \widehat{y_{t}}(t, 0)\right)=V(t, \widehat{x}(t))=V(t, 0),
$$

uma vez que $\widehat{x}(t)$ é tal que $\widehat{x}(t)(\tau)=0$ para todo $\tau$ (veja o Teorema 3.5), isto é, $\widehat{x}(t) \equiv 0$. Pela condição (ii), para $a=2 \max \{\|\psi\|,\|\bar{\psi}\|\}$, temos

$$
\begin{aligned}
\left|V\left(t, x_{\psi}(t)\right)-V\left(t, \bar{x}_{\bar{\psi}}(t)\right)\right| & =\left|U\left(t, y_{t}(t, \psi)\right)-U\left(t, \bar{y}_{t}(t, \bar{\psi})\right)\right| \\
& =|U(t, \psi)-U(t, \bar{\psi})| \\
& \leq K_{a}\|\psi-\bar{\psi}\|=K_{a}\left\|x_{\psi}(t)-\bar{x}_{\bar{\psi}}(t)\right\|
\end{aligned}
$$

onde aplicamos a Observação 4.1 para obter a última igualdade.

É claro que, dados $t \geq t_{0}$ e $z, \bar{z} \in B_{a}$, existem soluções $x$ e $\bar{x}$ da EDO generalizada (3.20) e funções $\psi, \bar{\psi} \in G^{-}\left([-r, 0], \mathbb{R}^{n}\right)$ tais que $z=x_{\psi}(t),\left(x_{\psi}(t)\right)_{t}=y_{t}(t, \psi), \bar{z}=\bar{x}_{\bar{\psi}}(t)$ e $\left(\bar{x}_{\bar{\psi}}(t)\right)_{t}=\bar{y}_{t}(t, \bar{\psi})$. Como

$$
\|\psi\|=\left\|y_{t}(t, \psi)\right\|=\left\|x_{\psi}(t)\right\|=\|z\|<a
$$

$\mathrm{e}$

$$
\|\bar{\psi}\|=\left\|\bar{y}_{t}(t, \bar{\psi})\right\|=\left\|\bar{x}_{\bar{\psi}}(t)\right\|=\|\bar{z}\|<a,
$$

pela Observação 4.1, então

$$
|V(t, z)-V(t, \bar{z})| \leq K_{a}\|z-\bar{z}\|, \quad z, \bar{z} \in B_{a} .
$$

Finalmente, como $t$ é arbitrário, o resultado segue.

Nos dois próximos resultados, nós daremos condições suficientes para que a solução do problema de valor inicial (4.1)-(4.2) seja uniformemente limitada e uniformemente eventualmente limitada respectivamente. 
Teorema 4.15 ([1], Teorema 5.4). Considere a EDFR impulsiva (4.1). Seja $U:\left[t_{0},+\infty\right) \times G^{-}\left([-r, 0], \mathbb{R}^{n}\right) \rightarrow$ $\mathbb{R}$ um funcional de Lyapunov. Suponha que U satisfaça as seguintes condições adicionais:

(i) $U(t, 0)=0, t \in\left[t_{0},+\infty\right)$;

(ii) Para cada $a>0$, existe uma constante $K_{a}>0$ tal que

$$
|U(t, \psi)-U(t, \bar{\psi})| \leq K_{a}\|\psi-\bar{\psi}\|, \quad t \in\left[t_{0},+\infty\right), \psi, \bar{\psi} \in \mathscr{B}_{a},
$$

onde $\mathscr{B}_{a}=\left\{\phi \in G^{-}\left([-r, 0], \mathbb{R}^{n}\right):\|\phi\|<a\right\} ;$

iii) A função $b: \mathbb{R}_{+} \rightarrow \mathbb{R}_{+}$da condição (ii) da Definição 4.2 é tal que $b(s) \rightarrow+\infty$ quando $s \rightarrow+\infty$.

Então a solução $y\left(t, t_{0}, \phi\right)$ de (4.1)-(4.2) será uniformemente limitada.

Demonstração. Note que, como $f$ satisfaz $(A)$ e $(B)$ e $I_{k}$ satisfaz $\left(A^{\prime}\right)$ e $\left(B^{\prime}\right)$ para $k=0,1,2, \ldots$, a função $G$ na equação (3.20) pertence à classe $\mathscr{F}(\Omega, h)$, onde $\Omega=P C_{1} \times\left[t_{0},+\infty\right)$ e $h=h_{1}+h_{2}$, com $h_{1}$ dada por (3.7) e $h_{2}$ dada por (3.14).

Considerando a função $V:\left[t_{0},+\infty\right) \times P C_{1} \rightarrow \mathbb{R}$ dada por (4.3), temos

$$
V(t, 0)=0
$$

e

$$
|V(t, x)-V(t, \bar{x})| \leq K_{a}\|x-\bar{x}\|,
$$

para $t \in\left[t_{0},+\infty\right)$ e $x, \bar{x} \in B_{a}$, pelo Lema 4.14

Portanto, a função $V$ satisfaz as condições $(i)$ e (ii) e do Teorema 2.11. Além disso, pela Observação 4.3, $V$ é um funcional de Lyapunov e é fácil ver que a condição (iii) do Teorema 2.11 se dá pela condição (iii) do presente teorema. Logo, pelo Teorema 2.11, $x$ é uniformemente limitada.

Agora, seja $y:\left[t_{0}-r,+\infty\right) \rightarrow \mathbb{R}^{n}$ a solução do problema de valor inicial (4.1)-(4.2) e seja $x$ a solução, em $\left[t_{0},+\infty\right)$, da EDO generalizada (3.20) dada pelo Teorema 3.5, satisfazendo a condição inicial $x\left(t_{0}\right)=$ $\widetilde{x}$, onde

$$
\widetilde{x}(\tau)=\left\{\begin{array}{l}
\phi\left(\tau-t_{0}\right), t_{0}-r \leq \tau \leq t_{0} \\
\phi(0), \tau \geq t_{0}
\end{array}\right.
$$


Então $x$ pode ser escrita como

$$
x(s)(\tau)=\left\{\begin{array}{l}
y(\tau), t_{0}-r \leq \tau \leq s \\
y(s), \tau \geq s
\end{array}\right.
$$

para $s \geq t_{0}$.

Seja $\alpha>0$ tal que

$$
\|\phi\|<\alpha
$$

Note que

$$
\|\widetilde{x}\|=\sup _{t_{0}-r \leq \tau<+\infty}\|\widetilde{x}(\tau)\|=\|\phi\|,
$$

por (4.39). Portanto (4.41) e (4.42) implicam

$$
\|\widetilde{x}\|<\alpha
$$

Sendo $x$ uma solução uniformemente limitada, existe $M>0$ tal que

$$
\|x(t)\|<M, \text { para qualquer } t \geq t_{0} .
$$

Portanto $\|x(t)\|<M$, para todo $t \in\left[t_{0}, b\right]$, onde $b$ é qualquer elemento em $\left(t_{0},+\infty\right)$. Em particular, $\|x(b)\|<M$. Mas (4.40) implica que, para qualquer $t \in\left[t_{0}, b\right]$,

$$
\begin{aligned}
|y(t)| \leq\left\|y_{t}\right\| & =\sup _{-r \leq \theta \leq 0}|y(t+\theta)| \leq \sup _{t_{0}-r \leq \tau \leq b}|y(\tau)| \\
& =\sup _{t_{0}-r \leq \tau \leq b}|x(b)(\tau)|=\|x(b)\|<M .
\end{aligned}
$$

Portanto a solução $y(t)=y\left(t, t_{0}, \phi\right)$ de (4.1)-(4.2) é uniformemente limitada e a prova está completa.

Teorema 4.16 ([1], Teorema 5.5). Considere a EDFR impulsiva (4.1) e suponha que $U:\left[t_{0},+\infty\right) \times$ $G^{-}\left([-r, 0], \mathbb{R}^{n}\right) \rightarrow \mathbb{R}$ seja um funcional de Lyapunov. Suponha, também, que U satisfaça as condições (i) até (iii) do Teorema 4.15 e que exista uma função contínua $\Lambda: \mathbb{R}^{+} \rightarrow \mathbb{R}^{+}$que satisfaça $\Lambda(0)=0$ e $\Lambda(x)>0$ se $x \neq 0$ tal que para toda $\psi \in G^{-}\left([-r, 0], \mathbb{R}^{n}\right)$, tenhamos

$$
D^{+} U(t, \psi) \leq-\Lambda(\|\psi\|), \quad t \geq t_{0}
$$

Então a solução $y(t)=y\left(t, t_{0}, \phi\right)$ de (4.1)-(4.2) será uniformemente eventualmente limitada. 
Demonstração. Considere $V:\left[t_{0},+\infty\right) \times P C_{1} \rightarrow \mathbb{R}$ dada por (4.3). Então as hipóteses do Teorema 2.11 estão satisfeitas.

Definamos $\Phi: P C_{1} \rightarrow \mathbb{R}$ por

$$
\Phi(z)=\Lambda(\|z\|), \quad z \in P C_{1} .
$$

Então $\Phi$ é contínua, $\Phi(0)=0$ e $\Phi(z)>0$, para $z \neq 0$.

Suponhamos que $x:[t,+\infty) \rightarrow G^{-}\left([t-r,+\infty), \mathbb{R}^{n}\right)$ seja uma solução de (3.20) tal que $(x(t))_{t}=\psi$, onde $t \in\left[t_{0},+\infty\right)$, e consideremos que $y:[t-r,+\infty) \rightarrow \mathbb{R}^{n}$ seja a solução de (4.1), com $y_{t}=\psi$, dada pelo Teorema 3.5. Por (4.4) e (4.46), temos

$$
\dot{V}\left(t, x_{\psi}(t)\right)=D^{+} U\left(t, y_{t}\right)=D^{+} U(t, \psi) \leq-\Lambda(\|\psi\|)=-\Lambda\left(\left\|y_{t}\right\|\right)
$$

Além disso, pela Obervação 4.1, temos

$$
\left\|y_{t}\right\|=\left\|x_{\psi}(t)\right\|
$$

Portanto,

$$
\dot{V}\left(t, x_{\psi}(t)\right) \leq-\Lambda\left(\left\|y_{t}\right\|\right)=-\Lambda\left(\left\|x_{\psi}(t)\right\|\right)=-\Phi\left(x_{\psi}(t)\right)
$$

e as hipóteses do Teorema 2.12 estão satisfeitas. Portanto, pelo Teorema 2.12, $x$ é uniformemente eventualmente limitada.

Agora, seja $y:\left[t_{0}-r,+\infty\right) \rightarrow \mathbb{R}^{n}$ a solução do sistema (4.1)-(4.2) e seja $x$ a solução em $\left[t_{0},+\infty\right)$ da EDO generalizada (3.20), dada pelo Teorema 3.5, satisfazendo a condição inicial $x\left(t_{0}\right)=\widetilde{x}$, onde

$$
\widetilde{x}(\tau)=\left\{\begin{array}{l}
\phi\left(\tau-t_{0}\right), t_{0}-r \leq \tau \leq t_{0} \\
\phi(0), \tau \geq t_{0}
\end{array}\right.
$$

Então, para $s \geq t_{0}, x$ pode ser escrita como

$$
x(s)(\tau)=\left\{\begin{array}{l}
y(\tau), t_{0}-r \leq \tau<s \\
y(s), \tau \geq s
\end{array}\right.
$$

Sendo $x$ uma solução uniformemente eventualmente limitada, existe $B>0$ tal que para todo $\alpha>0$, existe 
$T=T(\alpha)>0$ tal que se

$$
\|\widetilde{x}\|<\alpha
$$

então

$$
\|x(t)\|<B, \quad \text { para qualquer } t \geq t_{0}+T \text {. }
$$

Suponhamos que

$$
\|\phi\|<\alpha
$$

Precisamos provar que

$$
|y(t)|<B, \quad \text { para qualquer } t \geq t_{0}+T \text {. }
$$

Mas, isto segue imediatamente como na prova do Teorema 4.15. Por (4.51), obtemos (4.49) como em (4.42). Finalmente vale (4.52), já que temos (4.45) como no Teorema 4.15 e vale (4.50). Desta forma, finalizamos a prova.

\subsubsection{Exemplo}

Em [7], o autor estuda a equação

$$
y^{\prime}(t)=-\int_{t-r}^{t} p(t-s) g(y(s)) d s
$$

na teoria de um reator nuclear de circulação de combustível. Em tal modelo, $y$ é a densidade de nêutrons. É sabido que este é um bom modelo de viscoelasticidade unidimensional em que $y$ é a tensão e $p$ é a função de relaxamento.

Vamos considerar a equação (4.53) com condição de impulso e, posteriormente, aplicando a teoria desenvolvida, vamos obter a limitação uniforme da solução dessa equação. Considere, pois, a EDFR com ação impulsiva

$$
\begin{cases}y^{\prime}=-\int_{t-r}^{t} p(t-s) g(y(s)) d s, & t \neq \tau_{k}(y(t)), \quad t \geq 0 \\ \Delta y(t)=d_{k}, & t=\tau_{k}(y(t)), \quad k=1,2, \ldots\end{cases}
$$


com condição inicial

$$
y_{0}=\phi
$$

onde $r>0, \phi \in G^{-}\left([-r, 0], \mathbb{R}^{n}\right), p: \mathbb{R} \rightarrow \mathbb{R}_{+}$é uma função localmente Lebesgue integrável tal que $p(u) \leq B$ para todo $u \in \mathbb{R}, B$ é uma constante positiva, $g: \mathbb{R} \rightarrow \mathbb{R}_{+}$é tal que $|g(x)-g(y)| \leq H|x-y|$ para quaisquer $x, y \in \mathbb{R}, H>0$ e existe uma função $m: \mathbb{R} \rightarrow \mathbb{R}$ localmente Lebesgue integrável tal que

$$
\left|\int_{s_{1}}^{s_{2}} g(y(s)) d s\right| \leq \int_{s_{1}}^{s_{2}} m(s) d s
$$

para quaisquer $s_{1}, s_{2} \in \mathbb{R}$.

Consideraremos que 0 não é um momento de impulso do sistema (4.54)-(4.55), $\left\{d_{k}\right\}$ é uma sequência de constantes não positivas limitada inferiormente e que, para cada $k=1,2 \ldots, \tau_{k}$ é uma função contínua de $\mathbb{R}$ em $(0,+\infty)$. Além disso, consideraremos que as superfícies $\tau_{k}$ satisfazem as condições $(C 1)$ até (C5) do Capítulo 3.

Suponhamos que $\Delta y(t):=y(t+)-y(t-)=y(t+)-y(t)$, para qualquer $t \geq 0$. É fácil constatar que os operadores $I_{k}: \mathbb{R}^{n} \rightarrow \mathbb{R}^{n}$ dados por $I_{k}(y)=d_{k}$, para $y \in \mathbb{R}^{n}$ e $k=1,2, \ldots$, satisfazem as condições $\left(A^{\prime}\right)$ e $\left(B^{\prime}\right)$, para $k=1,2, \ldots$

Para cada $t \geq 0$, seja $f\left(y_{t}, t\right)=-\int_{t-r}^{t} p(t-s) g(y(s)) d s$. Vamos mostrar que $f$ satisfaz as condições $(A)$ e $(B)$ do Capítulo 3.

(A) Dados $y \in P C_{1}$ e $u_{1}, u_{2} \in[0,+\infty)$ com $u_{1} \leq u_{2}$, temos

$$
\begin{gathered}
\left|\int_{u_{1}}^{u_{2}} f\left(y_{s}, s\right) d s\right|=\left|\int_{u_{1}}^{u_{2}}\left(-\int_{s-r}^{s} p(s-u) g(y(u)) d u\right) d s\right| \leq \\
\leq \int_{u_{1}}^{u_{2}}\left(\int_{s-r}^{s}|p(s-u) g(y(u))| d u\right) d s \leq B \int_{u_{1}}^{u_{2}}\left|\int_{s-r}^{s} g(y(u)) d u\right| d s \leq \\
\leq B \int_{u_{1}}^{u_{2}}\left(\int_{s-r}^{s} m(u) d u\right) d s=\int_{u_{1}}^{u_{2}} M(s) d s,
\end{gathered}
$$

onde $M(s)=B \int_{s-r}^{s} m(u) d u$. Logo, a condição $(A)$ está satisfeita.

(B) Dados $x, y \in P C_{1}$ e $u_{1}, u_{2} \in[0,+\infty)$, temos

$$
\left|\int_{u_{1}}^{u_{2}} f\left(x_{s}, s\right)-f\left(y_{s}, s\right) d s\right|=\left|\int_{u_{1}}^{u_{2}}\left(-\int_{s-r}^{s} p(s-u)(g(x(u))-g(y(u))) d u\right) d s\right| \leq
$$




$$
\begin{gathered}
\leq\left|\int_{u_{1}}^{u_{2}}\left(\int_{s-r}^{s}|p(s-u)| z|x(u)-y(u)| d u\right) d s\right|= \\
=\left|\int_{u_{1}}^{u_{2}}\left(\int_{r}^{0}|p(\tau)| H|x(s-\tau)-y(s-\tau)| d \tau\right) d s\right| \leq \\
\leq \int_{u_{1}}^{u_{2}} H\left\|x_{s}-y_{s}\right\|\left(\int_{0}^{r}|p(\tau)| d \tau\right) d s \leq \\
\leq \int_{u_{1}}^{u_{2}} B H r\left\|x_{s}-y_{s}\right\| d s=\int_{u_{1}}^{u_{2}} L(s)\left\|x_{s}-y_{s}\right\| d s,
\end{gathered}
$$

onde $L(s)=B H r$, donde $f$ satisfaz $(B)$.

Definamos uma função $W: \mathbb{R} \rightarrow \mathbb{R}$ por $W(y)=\frac{y^{3}}{3}$. Seja $y$ uma solução de (4.54).

Para $t \neq t_{k}^{i}$, temos

$$
D^{+} W(y(t))=W^{\prime}(y(t)) y^{\prime}(t)=y^{2}(t) y^{\prime}(t)=-y^{2}(t) \int_{t-r}^{t} p(t-s) g(y(s)) d s \leq 0,
$$

uma vez que $p$ e $g$ são funções não negativas.

Note que $W\left(y\left(t_{k}^{i}+\right)\right)=W\left(y\left(t_{k}^{i}\right)+d_{k}\right) \leq W\left(y\left(t_{k}^{i}\right)\right)$, já que $W$ é uma função crescente e $d_{k} \leq 0$. Então, para $\eta>0$ suficientemente pequeno, temos $W\left(y\left(t_{k}^{i}+\eta\right)\right) \leq W\left(y\left(t_{k}^{i}\right)\right)$, pela continuidade de $W$. Portanto, para $t=t_{k}^{i}$, temos

$$
D^{+} W(y(t))=\limsup _{\eta \rightarrow 0^{+}} \frac{W(y(t+\eta))-W(y(t))}{\eta} \leq 0 .
$$

Agora, definamos uma função $U:[0,+\infty) \times G^{-}\left([-r, 0], \mathbb{R}^{n}\right) \rightarrow \mathbb{R}_{+}$por

$$
U(t, \psi)=\sup _{-r \leq \theta \leq 0} W(\psi(\theta))=\frac{1}{3} \sup _{-r \leq \theta \leq 0} \psi^{3}(\theta)=\frac{1}{3}\left(\sup _{-r \leq \theta \leq 0} \psi(\theta)\right)^{3}=\frac{1}{3}\|\psi\|^{3} .
$$

Mostraremos que $U$ é um funcional de Lyapunov e satisfaz as condições do Teorema 4.15.

(i) Pela definição de $U$, é claro que $U(t, 0)=0$ para todo $t \geq 0$.

(ii) Sejam $\psi, \varphi \in \mathscr{B}_{\rho}=\left\{\Psi \in G^{-}\left([-r, 0], \mathbb{R}^{n}\right):\|\Psi\|<\rho\right\}$. Então

$$
|U(t, \psi)-U(t, \varphi)| \leq \frac{1}{3}|\|\psi\|-\|\phi\||\left[\|\psi\|^{2}+\|\varphi\|\|\psi\|+\|\varphi\|^{2}\right]<\rho^{2}\|\psi-\varphi\|,
$$

para todo $t \geq 0$. 
(iii) Dados $t \geq 0$ e uma função $\psi \in G^{-}\left([-r, 0], \mathbb{R}^{n}\right)$, temos

$$
U(t, \psi)=\frac{1}{3}\|\psi\|^{3}=b(\|\psi\|)
$$

onde $b(s)=\frac{s^{3}}{3}$ é tal que $b(s) \rightarrow+\infty$ quando $s \rightarrow+\infty$.

(iv) Dados $t \geq 0$ e $\psi \in G^{-}\left([-r, 0], \mathbb{R}^{n}\right)$, seja $y$ a solução de (4.54) definida em $[t-r,+\infty]$ tal que $y_{t}=\psi$. Temos

$$
U(t, \psi)=U\left(t, y_{t}\right)=\sup _{-r \leq \theta \leq 0} W\left(y_{t}(\theta)\right)=W\left(y_{t}\left(\theta_{0}\right)\right)
$$

Queremos provar que $D^{+} U\left(t, y_{t}\right) \leq 0$. Para isto, vamos considerar dois casos: quando $\theta_{0}=0$ e o caso contrário. Inicialmente, suporemos que $\theta_{0}=0$. Neste caso,

$$
D^{+} U\left(t, y_{t}\right)=D^{+} W(y(t)) \leq 0
$$

Agora, consideremos $-r \leq \theta_{0}<0$. Como $\sup _{-r \leq \theta \leq 0} W\left(y_{t}(\theta)\right)=W\left(y_{t}\left(\theta_{0}\right)\right)$, para $\eta>0$ suficientemente pequeno, obtemos

$$
\sup _{-r \leq \theta \leq 0} W\left(y_{t+\eta}(\theta)\right)=\sup _{-r \leq \theta \leq 0} W\left(y_{t}(\theta)\right)=W\left(y_{t}\left(\theta_{0}\right)\right)
$$

Consequentemente,

$$
D^{+} U\left(t, y_{t}\right)=\limsup _{\eta \rightarrow 0^{+}} \frac{\sup _{-r \leq \theta \leq 0} W\left(y_{t+\eta}(\theta)\right)-\sup _{-r \leq \theta \leq 0} W\left(y_{t}(\theta)\right)}{\eta}=0 .
$$

Portanto o Teorema 4.15 implica que a solução única $y(t)=y(t, 0, \phi)$ de (4.54)-(4.55) é uniformente limitada. 
CAPÍTULO

5

\section{Apêndice: Propriedades qualitativas de soluções de EDFRs}

Neste capítulo complementar, relembraremos algumas propriedades qualitativas de soluções de EDFRs sem ocorrência de impulsos. Começaremos com conceitos básicos de EDFRs e enunciaremos resultados fundamentais para o desenvolvimento da teoria de EDFRs.

A principal referência para este capítulo é o clássico livro de J. Hale e S. M. Verduyn Lunel, [15].

\subsection{Noções e resultados básicos}

Sejam $I$ um intervalo limitado de $\mathbb{R}, \mathbb{R}^{n}$ o espaço vetorial $n$-dimensional sobre os reais com norma denotada por $|\cdot|$ e $C\left(I, \mathbb{R}^{n}\right)$ o espaço de Banach das funções contínuas de $I$ em $\mathbb{R}^{n}$, munido da topologia da convergência uniforme. Seja $r>0$. Então $C=C\left([-r, 0], \mathbb{R}^{n}\right)$ denota o espaço de Banach das funções contínuas de $[-r, 0]$ em $\mathbb{R}^{n}$ com a norma

$$
\|\phi\|=\sup _{-r \leq \theta \leq 0}|\phi(\theta)|
$$

para $\phi \in C$.

Seja $V$ um subconjunto aberto de $C \times \mathbb{R}$. Então $C\left(V, \mathbb{R}^{n}\right)$ é o espaço das funções contínuas de $V$ em $\mathbb{R}^{n}$. Denotaremos por $C^{0}\left(V, \mathbb{R}^{n}\right)$ o espaço das funções contínuas e limitadas de $V$ em $\mathbb{R}^{n}$. O espaço $C^{0}\left(V, \mathbb{R}^{n}\right)$ será um espaço de Banach quando estiver munido da norma induzida por $C\left(V, \mathbb{R}^{n}\right)$.

$\operatorname{Dados} t_{0} \in \mathbb{R}, \sigma>0$ e $x \in C\left(\left[t_{0}-r, t_{0}+\sigma\right], \mathbb{R}^{n}\right)$, para cada $t \in\left[t_{0}, t_{0}+\sigma\right]$, definimos $x_{t} \in C\left([-r, 0], \mathbb{R}^{n}\right)$ 
por

$$
x_{t}(\theta)=x(t+\theta), \quad-r \leq \theta \leq 0 .
$$

Definição 5.1. Sejam $\Omega \subseteq C \times \mathbb{R}$ um aberto e $f: \Omega \rightarrow \mathbb{R}^{n}$ uma função. Dizemos que a equação

$$
x^{\prime}(t)=f\left(x_{t}, t\right), \quad\left(x^{\prime}=\frac{d x}{d t}\right)
$$

é uma equação diferencial funcional retardada sobre $\Omega$.

Agora, apresentaremos o conceito de solução da equação (5.1).

Definição 5.2. Uma solução da equação (5.1) é uma função $x \in C\left(\left[t_{0}-r, t_{0}+\sigma\right], \mathbb{R}^{n}\right)$, onde $t_{0} \in \mathbb{R} e$ $\sigma>0$, tal que $\left(x_{t}, t\right) \in \Omega$ ex(t) satisfaz a equação (5.1), para $t \in\left[t_{0}, t_{0}+\sigma\right]$.

Dados $\phi \in C$ e $t_{0} \in \mathbb{R}$, diremos que $x\left(t_{0}, \phi, f\right)$ é uma solução da equação (5.1) com valor inicial $\phi$ em $t_{0}$, ou simplesmente uma solução passando por $\left(t_{0}, \phi\right)$, se existir $\sigma>0$ tal que $x\left(t_{0}, \phi, f\right)$ é uma solução da equação (5.1) sobre $\left[t_{0}-r, t_{0}+\sigma\right]$ e $x_{t_{0}}\left(t_{0}, \phi, f\right)=\phi$.

Com as notações e terminologias acima, enunciaremos resultados básicos da teoria de EDFRs, os quais estão presentes em [15].

Lema 5.3 ([15], Lema 2.1.1). Sejam $t_{0} \in \mathbb{R} e \phi \in C$. Se $f(\phi, t)$ for contínua, então encontrar uma solução da equação (5.1) passando por $\left(t_{0}, \phi\right)$ será equivalente a resolver a equação integral

$$
\begin{gathered}
x_{t_{0}}=\phi \\
x(t)=\phi(0)+\int_{t_{0}}^{t} f\left(x_{s}, s\right) d s, \quad t \geq t_{0} .
\end{gathered}
$$

Lema 5.4 ([15], Lema 2.2.1). Se $x \in C\left(\left[t_{0}-r, t_{0}+\alpha\right], \mathbb{R}^{n}\right)$, onde $\alpha>0$, então $x_{t}$ será uma função contínua como função de $t$ para $t \in\left[t_{0}, t_{0}+\alpha\right]$.

O próximo teorema trata da existência local de uma solução da EDFR (5.1). A prova deste resultado é decorrente do Teorema do Ponto Fixo de Schauder e pode ser encontrada em [15], página 43.

Teorema 5.5 (Existência local - [15], Teorema 2.2.1). Suponha que $\Omega$ seja um subconjunto aberto de $C \times \mathbb{R}$ e $f \in C\left(\Omega, \mathbb{R}^{n}\right)$. Se $\left(\phi, t_{0}\right) \in \Omega$, então existirá uma solução local de (5.1) por $\left(t_{0}, \phi\right)$. Mais geralmente, se $W \subseteq \Omega$ for compacto e $f \in C\left(\Omega, \mathbb{R}^{n}\right)$, então existirá uma vizinhança $V \subseteq \Omega$ de W tal que $f \in C^{0}\left(V, \mathbb{R}^{n}\right)$, e existirão uma vizinhança $U \subseteq C^{0}\left(V, \mathbb{R}^{n}\right)$ de $f$ e $\alpha>0$ tal que, para $\left(\phi, t_{0}\right) \in W$ e $g \in U$, existirá uma solução $x\left(t_{0}, \phi, g\right)$ de (5.1) passando por $\left(t_{0}, \phi\right)$ em $\left[t_{0}-r, t_{0}+\alpha\right]$. 
O resultado seguinte garante a existência de uma única solução local de (5.1) passando por $\left(t_{0}, \phi\right)$.

Teorema 5.6 (Unicidade - [15], Teorema 2.2.3). Sejam $\Omega \subseteq C \times \mathbb{R}$ um aberto e $\left(\phi, t_{0}\right) \in \Omega$. Se $f: \Omega \rightarrow \mathbb{R}^{n}$ for contínua e $f(\phi, t)$ for localmente Lipschtiziana com relação a $\phi$ em $\Omega$, então existirá uma única solução de (5.1) passando por $\left(t_{0}, \phi\right)$.

Para a definição abaixo, vamos supor que $f$, na equação (5.1), seja uma função contínua.

Definição 5.7. Se $x$ for uma solução de (5.1) em um intervalo $\left[t_{0}, a\right), a>t_{0}$, diremos que $\widetilde{x}$ será uma continuação de $x$, se existir um $b>$ a tal que $\widetilde{x}$ está definida em $\left[t_{0}-r, b\right)$ e $\widetilde{x}$ coincidir com $x$ em $\left[t_{0}-r, a\right)$ e satisfizer a equação (5.1) em $\left[t_{0}, b\right)$. Uma solução x será não continuável, se tal continuação não existir, ou seja, se o intervalo $\left[t_{0}, a\right)$ for o intervalo maximal de existência da solução $x$.

Cabe mencionar que, a existência de uma solução não continuável segue do Lema de Zorn. Além disso, o intervalo maximal de existência deve ser aberto à direita.

Na sequência, enunciaremos resultados que tratam de continuação de soluções de (5.1). Solicitamos aos leitores que vejam, em [15], as demonstrações dos mesmos.

Teorema 5.8 ([15], Teorema 2.3.1). Suponha que $\Omega$ seja um subconjunto aberto de $C \times \mathbb{R}$ e que $f \in$ $C\left(\Omega, \mathbb{R}^{n}\right)$. Se x for uma solução não continuável da equação (5.1) em $\left[t_{0}-r, b\right)$, então, para todo conjunto compacto $W$ em $\Omega$, existirá $t_{W}$ tal que $\left(x_{t}, t\right) \notin W$ para $t_{W} \leq t<b$.

Corolário 5.9 ([15], Corolário 2.3.1). Suponha que $\Omega$ seja um subconjunto aberto de $C \times \mathbb{R}$ e que $f$ pertença ao espaço de funções $C\left(\Omega, \mathbb{R}^{n}\right)$. Se x for uma solução não continuável da equação (5.1) em $\left[t_{0}-r, b\right)$ e $W$ for o fecho do conjunto $\left\{\left(x_{t}, t\right): t_{0} \leq t<b\right\}$ em $C \times \mathbb{R}$, então se $W$ for compacto, existirá uma sequência de números reais $\left\{t_{k}\right\}$, com $t_{k} \rightarrow b-q$ uando $k \rightarrow \infty$, tal que $\left(x_{t_{k}}, t_{k}\right)$ tende a $\partial \Omega$ quando $k \rightarrow \infty$, onde $\partial \Omega$ denota a fronteira de $\Omega$. Se $r>0$, então existirá $\phi \in C$ tal que $(\phi, b) \in \partial \Omega e$ $\left(x_{t}, t\right) \rightarrow(\phi, b)$ quando $t \rightarrow b^{-}$.

Teorema 5.10 ([15], Teorema 2.3.2). Suponha que $\Omega$ seja um subconjunto aberto de $C \times \mathbb{R}$ e que $f$ : $\Omega \mapsto \mathbb{R}^{n}$ seja completamente contínua, ou seja, $f$ é contínua e leva conjuntos fechados e limitados de $\Omega$ em conjuntos limitados de $\mathbb{R}^{n}$. Suponha, também, que x seja uma solução não continuável da equação (5.1) em $\left[t_{0}-r, b\right)$. Então, para todo conjunto limitado e fechado $U$ em $C \times \mathbb{R}, U$ em $\Omega$, existirá $t_{U}$ tal que $\left(x_{t}, t\right) \notin U$ para $_{U} \leq t<b$. 


\subsection{Estabilidade de soluções}

Seja $f: C\left([-r, 0], \mathbb{R}^{n}\right) \times \mathbb{R} \rightarrow \mathbb{R}^{n}$ uma função contínua. Considere a EDFR

$$
x^{\prime}(t)=f\left(x_{t}, t\right)
$$

Esta seção será dedicada a resultados sobre estabilidade da solução trivial $\bar{x} \equiv 0$ de (5.2).

No que segue, suporemos que $f(0, t)=0$ para todo $t \in \mathbb{R}$, para que a função $\bar{x} \equiv 0$ seja, de fato, uma solução de (5.2).

Relembremos alguns conceitos básicos de estabilidade.

Definição 5.11. A solução trivial $\bar{x} \equiv 0$ de (5.2) será dita

(i) Estável, se para quaisquer $t_{0} \in \mathbb{R}$ e $\varepsilon>0$, existir $\delta=\delta\left(\varepsilon, t_{0}\right)>0$ tal que se $x\left(t_{0}, \phi, f\right)$ for uma solução de (5.2) tal que $x_{t_{0}}\left(t_{0}, \phi, f\right)=\phi e$

$$
\|\phi\|<\delta
$$

então

$$
\left\|x_{t}\left(t_{0}, \phi, f\right)\right\|<\varepsilon, \quad t \geq t_{0} .
$$

(ii) Uniformemente estável, se o número $\delta$ no item (i) for independente de $t_{0}$.

(iii) Uniformemente assintoticamente estável, se existir $\delta_{0}>0$ e para todo $\varepsilon>0$, existir $T=T(\varepsilon) \geq 0$ tal que se $x\left(t_{0}, \phi, f\right)$ for uma solução de (5.2) tal que $x_{t_{0}}\left(t_{0}, \phi, f\right)=\phi e$

$$
\|\phi\|<\delta_{0}
$$

então

$$
\left\|x_{t}\left(t_{0}, \phi, f\right)\right\|<\varepsilon, \quad t \geq t_{0}+T .
$$

Observação 5.12. Se y for uma solução qualquer de (5.2), então y será dita estável, se a solução nula da equação

$$
z^{\prime}(t)=f\left(z_{t}+y_{t}, t\right)-f\left(y_{t}, t\right)
$$

for estável. Os outros conceitos de estabilidade para y(t) são definidos de forma análoga. 


\subsubsection{Teoremas de Lyapunov}

Nesta subseção, apresentaremos um resultado de estabilidade para a solução $\bar{x} \equiv 0$ de (5.2) que generaliza o segundo método de Lyapunov para as EDOs clássicas. Lembremos que o segundo método de Lyapunov é um método usado para estudar a estabilidade sem fazer uso da forma explícita das soluções. Este método tenta obter informações diretamente pelo uso, juntamente com as equações diferenciais, de funções adequadas convenientemente chamadas de funcionais de Lyapunov.

Se $U: \mathbb{R} \times C\left([-r, 0], \mathbb{R}^{n}\right) \rightarrow \mathbb{R}$ for uma função contínua e $x(t, \phi, f)$ for uma solução de (5.2) passando por $(t, \phi)$, definiremos

$$
D^{+} U(t, \phi)=\limsup _{\eta \rightarrow 0+} \frac{U\left(t+\eta, x_{t+\eta}(t, \phi, f)\right)-U\left(t, x_{t}(t, \phi, f)\right)}{\eta}, \quad t \in \mathbb{R} .
$$

A função $D^{+} U(t, \phi)$ denota a derivada superior à direita de $U$ ao longo da solução $x(t, \phi, f)$ de (5.2).

A seguir, temos o conceito de funcional de Lyapunov com respeito a EDFR (5.2).

Definição 5.13. Diremos que $U: \mathbb{R} \times C\left([-r, 0], \mathbb{R}^{n}\right) \rightarrow \mathbb{R}$ é um funcional de Lyapunov (com respeito a EDFR (5.2)), se as seguintes condições forem satisfeitas:

(i) U é contínuo nas duas variáveis;

(ii) $U$ é não negativo, ou seja, $U(t, \phi) \geq 0$ para quaisquer $t \in \mathbb{R} e \phi \in C\left([-r, 0], \mathbb{R}^{n}\right)$;

(iii) $D^{+} U(t, \phi) \leq 0, \operatorname{com} D^{+} U(t, \phi)$ dada por (5.4).

O resultado abaixo foi extraído de [15]. Não consideramos pertinente incluir sua demonstração aqui.

Teorema 5.14 ([15], Teorema 5.2.1). Suponha que $f: C\left([-r, 0], \mathbb{R}^{n}\right) \times \mathbb{R} \rightarrow \mathbb{R}^{n}$ aplique subconjuntos limitados de $C\left([-r, 0], \mathbb{R}^{n}\right) \times \mathbb{R}$ em subconjuntos limitados de $\mathbb{R}^{n}$. Sejam $u, v, w: \mathbb{R}_{+} \rightarrow \mathbb{R}_{+}$funções contínuas e não decrescentes tais que $u(s)>0$ e $v(s)>0$ para $s>0$ e $u(0)=v(0)=0$. Se existir um funcional de Lyapunov $U: \mathbb{R} \times C\left([-r, 0], \mathbb{R}^{n}\right) \rightarrow \mathbb{R}$ tal que

$$
u(|\phi(0)|) \leq U(t, \phi) \leq v(\|\phi\|)
$$

$e$

$$
D^{+} U(t, \phi) \leq-w(|\phi(0)|)
$$


então a solução $\bar{x} \equiv 0$ da equação (5.2) será uniformemente estável. Se $w(s)>0$ para $s>0$, a solução $\bar{x} \equiv 0$ da equação (5.2) será uniformemente assintoticamente estável.

Em [15], após exibir um determinado funcional de Lyapunov, os autores afirmam que a solução nula da equação escalar abaixo é uniformemente assintoticamente estável. Exibimos uma prova deste fato, para facilitar a compreensão do leitor.

Exemplo 5.15. Considere a equação escalar

$$
x^{\prime}(t)=-a x(t)-b x(t-r) .
$$

Definamos

$$
U(\phi)=\frac{\phi^{2}(0)}{2}+\xi \int_{-r}^{0} \phi^{2}(\theta) d \theta, \quad \xi>0 .
$$

Então, considerando $x(t, \phi)$ uma solução de (5.5) tal que $x_{t}=x_{t}(t, \phi)=\phi$, temos

$$
\begin{gathered}
D^{+} U(\phi)=\limsup _{\eta \rightarrow 0+} \frac{U\left(x_{t+\eta}(t, \phi)\right)-U\left(x_{t}(t, \phi)\right)}{\eta}= \\
=\limsup _{\eta \rightarrow 0+} \frac{\frac{x_{t+\eta}^{2}(0)}{2}+\xi \int_{-r}^{0} x_{t+\eta}^{2}(\theta) d \theta-\frac{x_{t}^{2}(0)}{2}-\xi \int_{-r}^{0} x_{t}^{2}(\theta) d \theta}{\eta}= \\
=\left(\frac{x_{t}^{2}(0)}{2}\right)^{\prime}+\left(\xi \int_{-r}^{0} x_{t}^{2}(\theta) d \theta\right)^{\prime}=x(t) x^{\prime}(t)+\xi\left[x^{2}(t)-x^{2}(t-r)\right]= \\
=x(t)[-a x(t)-b x(t-r)]+\xi\left[x^{2}(t)-x^{2}(t-r)\right]= \\
=-(a-\xi) x_{t}^{2}(0)-b x_{t}(0) x_{t}(-r)-\xi x_{t}^{2}(-r) .
\end{gathered}
$$

Logo

$$
D^{+} U(\phi)=-(a-\xi) \phi^{2}(0)-b \phi(0) \phi(-r)-\xi \phi^{2}(-r) .
$$

É fácil checar o fato de que $D^{+} U(\phi) \leq 0$ se, e somente se,

$$
a>\xi>0, \quad 4(a-\xi) \xi>b^{2} .
$$

Tomemos $\xi=\frac{a}{2}$ e $a>0$. Note que

$$
\frac{\phi^{2}(0)}{2} \leq U(\phi) \leq \frac{1+a r}{2}\|\phi\|^{2} .
$$


Tomando $u(s)=\frac{s^{2}}{2}$ e $v(s)=\frac{1+a r}{2} s^{2}$, obtemos

$$
u(|\phi(0)|) \leq U(\phi) \leq v(\|\phi\|) .
$$

Claramente u e v são funções contínuas e não decrescentes tais que $u(s)>0$ se $s>0, v(s)>0$ se $s>0$ $e u(0)=v(0)=0$.

Além disso, para $\xi=\frac{a}{2}$ e $a>0$, temos $|b|<a$. Se $b=\frac{a}{2}$, então

$$
\begin{aligned}
-D^{+} U(\phi) & =\frac{a}{2} \phi^{2}(0)+\frac{a}{2} \phi(0) \phi(-r)+\frac{a}{2} \phi^{2}(-r) \\
& \geq \frac{a}{2} \phi^{2}(0)+\frac{a}{2} B^{2}+\frac{a}{2} B^{2} \\
& \geq \frac{a}{2} \phi^{2}(0)+a B^{2},
\end{aligned}
$$

onde $B=\inf _{\theta \in[-r, 0]} \phi(\theta)$. Tal B existe pois $\phi$ é uma função contínua definida no intervalo compacto $[-r, 0]$. Portanto,

$$
D^{+} U(\phi) \leq-\left(\frac{a}{2} \phi^{2}(0)+a B^{2}\right)=-w(|\phi(0)|),
$$

onde $w(s)=\frac{a}{2} s^{2}+a B^{2}$. Claramente $w$ é uma função contínua e não decrescente e $w(s)>0$, se $s>0$.

Portanto, para $\xi=\frac{a}{2}, a>0$ e $b=\frac{a}{2}$, as hipóteses do Teorema 5.14 estão satisfeitas. Logo a solução nula $\bar{x} \equiv 0$ da equação (5.5) é uniformemente assintoticamente estável.

\subsubsection{Teoremas de Razumikhin}

Na seção anterior, mostramos um estudo da estabilidade da solução nula de uma EDFR através do uso de funcionais de Lyapunov com respeito a tal EDFR. Porém, é mais natural explorar a possibilidade de utilizar a taxa de variação de uma função em $\mathbb{R}^{n}$ para determinar condições suficientes para a estabilidade de soluções. Resultados nesta direção são geralmente chamados de Teoremas do tipo Razumikhin. Portanto, agora, consideraremos funções $U: \mathbb{R} \times \mathbb{R}^{n} \rightarrow \mathbb{R}$ e apresentaremos outros resultados de estabilidade para a solução trivial da EDFR (5.2). Não exibiremos as provas dos resultados expostos aqui. Para a verificação destas, indicamos a referência [15].

Se $U: \mathbb{R} \times \mathbb{R}^{n} \rightarrow \mathbb{R}$ for uma função contínua, então $D^{+} U(t, \phi(0))$, a derivada de $U$ ao longo das soluções de (5.2) será definida por

$$
D^{+} U(t, \phi(0))=\limsup _{\eta \rightarrow 0+} \frac{U(t+\eta, x(t, \phi, f)(t+\eta))-U(t, \phi(0))}{\eta}
$$


onde $x(t, \phi, f)(s)$ é a solução da EDFR (5.2) passando por $(t, \phi)$.

Definição 5.16. Diremos que $U: \mathbb{R} \times \mathbb{R}^{n} \rightarrow \mathbb{R}$ é uma função de Lyapunov (com respeito a EDFR (5.2)), se U satisfizer as seguintes condições:

(i) U é uma função contínua nas duas variáveis;

(ii) $U$ é uma função não negativa, ou seja, $U(t, x) \geq 0$ para quaisquer $t \in \mathbb{R}$ e $x \in \mathbb{R}^{n}$;

(iii) $D^{+} U(t, \phi(0)) \leq 0$, com $D^{+} U(t, \phi(0))$ dada por (5.6).

Teorema 5.17 ([15], Teorema 5.4.1). Suponha que $f: C\left([-r, 0], \mathbb{R}^{n}\right) \times \mathbb{R} \rightarrow \mathbb{R}^{n}$ aplique subconjuntos limitados de $C\left([-r, 0], \mathbb{R}^{n}\right) \times \mathbb{R}$ em subconjuntos limitados de $\mathbb{R}^{n}$ e considere a EDFR (5.2). Sejam $u, v, w: \mathbb{R}_{+} \rightarrow \mathbb{R}_{+}$funções contínuas e não decrescentes tais que $u(s)>0$ e $v(s)>0$ para $s>0, u(0)=$ $v(0)=0$ e v é crescente. Se existir uma função de Lyapunov $U: \mathbb{R} \times \mathbb{R}^{n} \rightarrow \mathbb{R}$ com respeito a EDFR (5.2) tal que

$$
u(|x|) \leq U(t, x) \leq v(|x|), \quad t \in \mathbb{R}, x \in \mathbb{R}^{n},
$$

$e$

$$
D^{+} U(t, \phi(0)) \leq-w(|\phi(0)|), \quad \text { se } U(t+\theta, \phi(\theta)) \leq U(t, \phi(0)),
$$

para $\phi \in C\left([-r, 0], \mathbb{R}^{n}\right)$ e $\theta \in[-r, 0]$, então a solução $\bar{x} \equiv 0$ da equação (5.2) será uniformemente estável.

Teorema 5.18 ([15], Teorema 5.4.2). Suponha que todas as condições do Teorema 5.17 estejam satisfeitas. Suponha, ainda, que $w(s)>0$ para $s>0$. Se existir uma função contínua e não decrescente $p(s)>$ s para $s>0$ de modo que a condição (5.8) seja substituída por

$$
D^{+} U(t, \phi(0)) \leq-w(|\phi(0)|), \quad \text { se } U(t+\theta, \phi(\theta))<p(U(t, \phi(0))
$$

para $\phi \in C\left([-r, 0], \mathbb{R}^{n}\right)$ e $\theta \in[-r, 0]$, então a solução $\bar{x} \equiv 0$ da equação (5.2) será uniformemente assintoticamente estável.

O próximo exemplo foi extraído de [15], Capítulo 5.

Exemplo 5.19. Consideremos a equação

$$
x^{\prime}=-a(t) x(t)-b(t) x\left(t-r_{0}(t)\right)
$$

onde $a$, $b$ e $r_{0}$ são funções contínuas e limitadas em $\mathbb{R}$, com $|b(t)| \leq a(t)$ e $0 \leq r_{0}(t) \leq r$, para todo 
$t \in \mathbb{R} . \operatorname{Se} U(x)=\frac{x^{2}}{2}$, então

$$
\begin{aligned}
D^{+} U(x(t)) & =x(t) x^{\prime}(t)=-a(t) x^{2}(t)-b(t) x(t) x\left(t-r_{0}(t)\right) \\
& \leq-a(t) x^{2}(t)+|b(t)||x(t)|\left|x\left(t-r_{0}(t)\right)\right| \\
& \leq-(a(t)-|b(t)|) x^{2}(t) \leq 0,
\end{aligned}
$$

se $|x(t)| \geq \mid x\left(t-r_{0}(t) \mid\right.$. Como $U(x)=\frac{x^{2}}{2}$, então $D^{+} U(x(t)) \leq 0$, se $U(x(t)) \geq U\left(x\left(t-r_{0}(t)\right)\right.$. Além disso, é fácil verificar que (5.7) vale. Portanto o Teorema 5.17 implica que a solução $\bar{x} \equiv 0$ de (5.9) é uniformemente estável.

Se, além disso, tivermos $a(t) \geq \delta>0$, e existir $k, 0 \leq k<1$ tal que $|b(t)| \leq k \delta$, então a solução $\bar{x} \equiv 0$ de (5.9) será uniformemente assintoticamente estável. Com efeito, considere $p(s)=q^{2}$ s para alguma constante $q>1$. Se $U(x)=\frac{x^{2}}{2}$, então

$$
D^{+} U(x(t)) \leq-(1-q k) \delta x^{2}(t)
$$

se $p(U(x(t))) \geq U\left(x\left(t-r_{0}(t)\right)\right.$. Como $k<1$, existe $q>1$ tal que $1-q k>0$. Desta forma, o Teorema 5.18 implica a estabilidade uniforme assintótica da solução nula de (5.9).

\subsection{Limitação de soluções}

Nesta seção, apresentaremos métodos para determinar limitação uniforme e limitação eventual uniforme de soluções de EDFRs.

Consideremos a EDFR

$$
x^{\prime}(t)=f\left(x_{t}, t\right) .
$$

com condição inicial

$$
x_{t_{0}}=\phi
$$

onde $f: C\left([-r, 0], \mathbb{R}^{n}\right) \times \mathbb{R} \rightarrow \mathbb{R}^{n}$ é uma função contínua e $\phi \in C\left([-r, 0], \mathbb{R}^{n}\right)$.

Seja $t_{0} \in \mathbb{R}$. Consideraremos a solução do problema de valor inicial (5.10)-(5.11) passando por $\left(t_{0}, \phi\right)$ e denotada por $x\left(t_{0}, \phi, f\right)$.

Relembremos alguns conceitos elementares sobre limitação de solução. 
Definição 5.20. A solução $x=x\left(t_{0}, \phi, f\right)$ do sistema (5.10)-(5.11) será dita

(i) Uniformemente limitada, se para todo $\alpha>0$, existir $M=M(\alpha)>0$ tal que se

$$
\|\phi\|<\alpha
$$

então

$$
|x(s)|<M, \quad s \geq t_{0} .
$$

(ii) Quase uniformemente eventualmente limitada, se existir uma constante B $>0$ tal que para todo $\alpha>0$, existe $T=T(\alpha)>0$ tal que se

$$
\|\phi\|<\alpha
$$

então

$$
|x(s)|<B, \quad s \geq t_{0}+T(\alpha) .
$$

(iii) Uniformemente eventualmente limitada, se for uniformemente limitada e quase uniformemente eventualmente limitada.

Funcionais de Lyapunov também são usados para dar condições suficientes para a limitação uniforme da solução do problema de valor inicial (5.10)-(5.11). Veja o próximo resultado.

Teorema 5.21 ([15], Teorema 5.2.1). Suponha que $f: C\left([-r, 0], \mathbb{R}^{n}\right) \times \mathbb{R} \rightarrow \mathbb{R}^{n}$ leve subconjuntos limitados de $C\left([-r, 0], \mathbb{R}^{n}\right) \times \mathbb{R}$ em subconjuntos limitados de $\mathbb{R}^{n}$. Sejam $u, v, w: \mathbb{R}_{+} \rightarrow \mathbb{R}_{+}$funções contínuas e não decrescentes tais que $u(s)>0$ e $v(s)>0$ para $s>0, u(0)=v(0)=0$ e $u(s) \rightarrow+\infty$ quando $s \rightarrow+\infty$. Se existir um funcional de Lyapunov $U: \mathbb{R} \times C\left([-r, 0], \mathbb{R}^{n}\right) \rightarrow \mathbb{R}$ com respeito a EDFR (5.10) tal que

$$
u(|\phi(0)|) \leq U(t, \phi) \leq v(\|\phi\|)
$$

$e$

$$
D^{+} U(t, \phi) \leq-w(|\phi(0)|)
$$

então a solução $x\left(t_{0}, \phi, f\right)$ do problema de valor inicial (5.10)-(5.11) será uniformemente limitada.

Note que $D^{+} U(t, \phi)$ é como em (5.4).

Observação 5.22. No Exemplo 5.15, a função $u(s)=\frac{s^{2}}{2}$ é tal que $u(s) \rightarrow+\infty$ quando $s \rightarrow+\infty$. Portanto, com as mesmas hipóteses sobre $a, b e \xi$, dados $t_{0} \in \mathbb{R} e \phi \in C\left([-r, 0], \mathbb{R}^{n}\right)$, concluímos, pelo Teorema 
5.21, que a solução da equação (5.5) que passa por $\left(t_{0}, \phi\right)$ é uniformemente limitada.

A seguir, veremos que um teorema do tipo Razumikhin nos dá condições suficientes para que a solução do problema de valor inicial (5.10)-(5.11) seja uniformemente eventualmente limitada. Consideraremos $D^{+} U(t, \phi(0))$ dada por (5.6).

Teorema 5.23 ([15], Teorema 5.4.3). Suponha que $f: C\left([-r, 0], \mathbb{R}^{n}\right) \times \mathbb{R} \rightarrow \mathbb{R}^{n}$ leve subconjuntos limitados de $C\left([-r, 0], \mathbb{R}^{n}\right) \times \mathbb{R}$ em subconjuntos limitados de $\mathbb{R}^{n}$ e considere a EDFR (5.10). Sejam $u, v, w: \mathbb{R}_{+} \rightarrow \mathbb{R}_{+}$funções contínuas e não decrescentes, com $u(s) \rightarrow+\infty$ quando $s \rightarrow+\infty$. Se existir uma função de Lyapunov $U: \mathbb{R} \times \mathbb{R}^{n} \rightarrow \mathbb{R}$ tal que

$$
u(|x|) \leq U(t, x) \leq v(|x|), \quad t \in \mathbb{R}, x \in \mathbb{R}^{n},
$$

$e$

$$
D^{+} U(t, \phi(0)) \leq-w(|\phi(0)|)
$$

sempre que

$$
|\phi(0)| \geq H \quad e \quad U(t+\theta, \phi(\theta)) \leq p(U(t, \phi(0)))
$$

para $\phi \in C\left([-r, 0], \mathbb{R}^{n}\right), \theta \in[-r, 0]$, onde $H$ é uma constante positiva, então a solução $x\left(t_{0}, \phi\right)(s)$ do problema de valor inicial (5.10)-(5.11) será uniformemente eventualmente limitada.

Segue, abaixo, uma aplicação do Teorema 5.23.

Exemplo 5.24. Consideremos o sistema de segunda ordem

$$
\begin{aligned}
& \dot{x}(t)=y(t) \\
& \dot{y}(t)=-\Phi(t, y(t))-f(x(t))+p(t)+\int_{-r}^{0} g(x(t+\theta)) y(t+\theta) d \theta .
\end{aligned}
$$

Se $g(x)=\frac{d f(x)}{d x}$, então o sistema (5.13) incluirá a equação escalar de segunda ordem

$$
\ddot{x}(t)+\Phi(t, \dot{x}(t))+f(x(t-r))=p(t) .
$$

Faremos as seguintes hipóteses sobre o sistema (5.13):

(i) $\Phi: \mathbb{R}^{2} \rightarrow \mathbb{R}$ é contínua, $\Phi$ leva $\mathbb{R} \times($ conjuntos limitados de $\mathbb{R}$ ) em conjuntos limitados de $\mathbb{R} e$ 
existem constantes $a>0$ e $H>0$, tais que

$$
\frac{\Phi(t, y)}{y}>a>0 \quad \text { para }|y| \geq H
$$

(ii) $f: \mathbb{R} \rightarrow \mathbb{R}$ é contínua e $f(x)$ sgn $x \rightarrow+\infty$ quando $|x| \rightarrow+\infty$, onde sgn $x=\left\{\begin{array}{l}+1, \text { se } x>0 \\ -1, \text { se } x<0\end{array}\right.$.

(iii) $g: \mathbb{R} \rightarrow \mathbb{R}$ é contínua e $|g(x)| \leq L$ para todo $x \in \mathbb{R}$;

(iv) $\operatorname{Lr}<a$.

Nestas condições, é possível mostrar que as soluções do sistema (5.13) são uniformemente eventualmente limitadas. Para a verificação deste fato, veja [15], página 160. 


\section{Referências Bibliográficas}

[1] S. M. Afonso; E. Bonotto; M. Federson; L. P. Gimenes, Boundedness of solutions of functional differential equations with variable impulses via generalized ordinary differential equations, submetido.

[2] S. M. Afonso; E. Bonotto; M. Federson; L. P. Gimenes, Stability of functional differential equations with variable impulsive perturbations via generalized ordinary differential equations, submetido.

[3] S. M. Afonso; E. Bonotto; M. Federson, On exponential stability of functional differential equations with variable impulsive perturbations, preprint.

[4] Z. Artstein, Topological dynamics of an ordinary differential equation and Kurzweil equations, $J$. Diff. Eq. 23 (1977), 224-243.

[5] J. F. M. Al-Omari; S. A. Gourley, Dynamics of a stage-structured population model incorporating a state-dependent maturation delay. Nonlinear Anal. Real World Appl. 6(1), (2005), 13-33.

[6] J. Cao; J. Wang, Global exponential stability and periodicity of recurrent neural networks with time delays. IEEE Trans. Circuits Syst. I Regul. Pap. 52(5), (2005), 920-931.

[7] W. K. Ergen, Kinetics of the circulating fuel nuclear reactor. J. Appl. Phys. 25(1954), 702-711.

[8] M. Federson; Š. Schwabik, Generalized ODEs approach to impulsive retarded differential equations. Differential and Integral Equations. 19(11), (2006), 1201-1234.

[9] M. Federson; Š. Schwabik, Stability for retarded differential equations, Ukr. Math. Journal 60, (2008), 107-126.

[10] M. Federson; Š. Schwabik, A new approach to impulsive retarded differential equations: stability results, Functional Differential Equations 16(4), (2009), 583-607.

[11] Fu, Xilin; Li, Xiaodi, Razumikhin-type theorems on exponential stability of impulsive infinite delay differential systems, Journal of Computational and Applied Mathematics 224, (2009), 1-10. 
[12] J. B. Godoy, Método da Média para Equações Diferenciais Funcionais Retardadas Impulsivas via Equações Diferenciais Generalizadas, Dissertação de Mestrado, ICMC-USP, 2009.

[13] K. Gopalsamy, Stability and Oscillations in Delay Differential Equations of Population Dynamics. Mathematics and its Applications, 74. Kluwer Academic Publishers Group, Dordrecht, 1992.

[14] I. Györi; G. Ladas, Oscillation Theory of Delay Differential Equations. With applications. Oxford Mathematical Monographs. Oxford Science Publications. The Clarendon Press, Oxford University Press, New York, 1991.

[15] J. K. Hale; S. M. Verduyn Lunel, Introduction to Functional Differential Equations. Applied Mathematical Sciences, 99. Springer-Verlag, New York, 1993.

[16] R. Henstock, Lectures on the Theory of Integration, World Scientific, Singapore, 1988.

[17] C. S. Hönig, Volterra Stieltjes Integral Equations, North Holland Publ. Comp., Amsterdam, 1975.

[18] J. J. Hopfield, Neural networks and physical systems with emergent collective computational abilities. Proc. Nat. Acad. Sci. U.S.A. 79(8), (1982), 2554-2558.

[19] E. Kreyszig, Introductory Functional Analysis with Aplications, Wiley, 1989.

[20] Y. Kuang, Delay Differential Equations with Applications in Population Dynamics. Mathematics in Science and Engineering, 191. Academic Press, Inc., Boston, MA, 1993.

[21] J. Kurzweil, Generalized ordinary differential equations and continuous dependence on a parameter, Czechoslovak Math. J. 7(82) (1957), 418-448.

[22] Xinzhi Liu; G. Ballinger, Uniform asymptotic stability of impulsive delay differential equations. Comput. Math. Appl. 41(7-8), (2001), 903-915.

[23] S. Mohamad, Global exponential stability in continuous-time and discrete-time delayed bidirectional neural networks. Phys. D 159(3-4), (2001), 233-251.

[24] S. H. Saker; S. Agarwal, Oscillation and global attractivity in a nonlinear delay periodic model of population dynamics. Appl. Anal. 81(4), (2002), 787-799.

[25] J. H. Shen, Razumikhin techniques in impulsive functional-differential equations. Nonlinear Anal. 36(1) (1999), 119-130. 
[26] Jianhua Shen; Jurang Yan, Razumikhin type stability theorems for impulsive functional-differential equations. Nonlinear Anal. 33(5), (1998), 519-531.

[27] Š. Schwabik, Generalized Ordinary Differential Equations, World Scientific, Series in Real Anal., vol. 5, 1992.

[28] Š. Schwabik, Variational stability for generalized ordinary differential equations, Časopis Pěst. Mat. 109(4), (1984), 389-420.

[29] I. M. Stamova, Boundedness of impulsive functional differential equations with variable impulsive perturbations. Bull. Austral. Math. Soc. 77(2), (2008), 331-345.

[30] Xu, Daoyi; Zhao, Hongyong; Zhu, Hong, Global dynamics of Hopfield neural networks involving variable delays. Comput. Math. Appl. 42(1-2), (2001), 39-45.

[31] Zhou, Dongming; Cao, Jinde, Globally exponential stability conditions for cellular neural networks with time-varying delays. Appl. Math. Comput. 131(2-3), (2002), 487-496. 


\section{Índice Remissivo}

correspondência entre EDFRs impulsivas e EDOs generalizadas, 53

\section{Equação}

diferencial funcional com retardo e impulsos em tempo variável, 50

diferencial funcional retardada, 102

diferencial ordinária generalizada, 19

função

de Lyapunov, 108

funcional de Lyapunov, 35, 72, 105

integral de Kurzweil, 16

Lema de Saks-Henstock, 17

solução

de uma EDFR, 102

de uma EDFR impulsiva , 51

de uma EDO generalizada, 19

estável, 74, 104

exponencialmente estável, 89

quase uniformemente eventualmente limitada,

$$
44,92
$$

uniformemente assintoticamente estável, 74, 104

uniformemente estável, 74, 104

uniformemente eventualmente limitada, 45, 92

uniformemente limitada, 44, 92

variacionalmente assintoticamente estável, 34

variacionalmente atratora, 34

variacionalmente estável, 34 variacionalmente exponencialmente estável, 42

\section{Teorema}

de existência local de solução de uma EDFR, 102

de existência local e unicidade de solução de uma EDFR impulsiva, 69

de existência local e unicidade de solução de uma EDO generalizada, 27

de unicidade de solução de uma EDFR, 103 